Portland State University

PDXScholar

Fall 1-8-2015

\title{
Developmental Perspectives on Motivational Resilience: Predictors of Eighth-grade At-risk Students' Academic Engagement and Achievement
}

Heather Anne Brule

Portland State University

Follow this and additional works at: https://pdxscholar.library.pdx.edu/open_access_etds

Part of the Educational Psychology Commons, Elementary and Middle and Secondary Education Administration Commons, and the School Psychology Commons Let us know how access to this document benefits you.

\section{Recommended Citation}

Brule, Heather Anne, "Developmental Perspectives on Motivational Resilience: Predictors of Eighth-grade At-risk Students' Academic Engagement and Achievement" (2015). Dissertations and Theses. Paper 2113.

https://doi.org/10.15760/etd.2111

This Thesis is brought to you for free and open access. It has been accepted for inclusion in Dissertations and Theses by an authorized administrator of PDXScholar. Please contact us if we can make this document more accessible: pdxscholar@pdx.edu. 
Developmental Perspectives on Motivational Resilience:

Predictors of Eighth-grade At-risk Students' Academic Engagement and Achievement

by

Heather Anne Brule

A thesis submitted in partial fulfillment of the requirements for the degree of

Master of Science

in

Psychology

Thesis Committee:

Ellen Skinner, chair

Robert Roeser

Joel Steele

Portland State University

2014 


\begin{abstract}
This study uses the concept of stage-environment fit Eccles et al., 1993) in conjunction with self-determination theory (Deci \& Ryan, 1985) to guide an investigation of at-risk eighth graders' motivational and academic resilience. A developmentally-calibrated method was used to divide students into motivational and academic resilience groups based on their resilient, average, or stress-affected levels of academic engagement and GPA. Data from 167 eighth graders and 155 sixth graders were used to examine the extent to which students' ratings of autonomy, teacher support, peer support, and engagement in gardenbased education were related to resilience group membership, and whether these four resources seemed more important to eighth graders than to sixth graders.

Results provided support for the overall hypothesis that the four developmental resources (autonomy, teacher support, peer support, and garden engagement) seemed to serve as motivational and/or academic resources, but did not, generally, provide support for their being more important for eighth graders than for sixth graders. Analyses of variance and regressions showed that autonomy, teacher support, and garden engagement were all resources for eighth graders' motivation and achievement, with autonomy and teacher support as the strongest resources. Peer support was only a resource for motivation, and only for eighth-grade boys.
\end{abstract}

In terms of unique effects, only autonomy uniquely predicted eighth 
graders' dual (motivational and academic) resilience group membership when controlling for the other developmental resources. In contrast, autonomy, teacher support, and garden engagement all uniquely predicted sixth graders' dual resilience group membership. Autonomy's status as the only unique predictor of eighth-grade resilience contrasted with an overall trend in which correlations among all variables were weaker for eighth graders than sixth graders. This contrast suggested that one part of eighth-grade risk might be an increased immunity to motivational resources.

Autonomy seemed to fully mediate the relationship between teacher support and dually-resilient group membership for eighth graders, highlighting autonomy's role as a key component in motivational processes for at-risk eighthgraders. The study shed light on the educational risk posed by "eighthgradeness" in conjunction with having an at-risk demographic status and a being traditional middle school setting, and suggested that autonomy might be a necessary, if not sufficient, route to eighth-grade motivational and academic resilience in light of this risk. 


\section{Acknowledgements}

I owe a great debt of gratitude to those people who contributed both to this project and to my professional and personal growth. First, I would like to thank the members of my thesis committee. I thank Robert Roeser for his expertise and patience in supporting this project, and for stoking my passion regarding both adolescence and the world in general. I thank Joel Steele for getting to the heart of things while supporting me both as a learner of statistics and as a whole person; in this he has gone above and beyond. I owe special thanks to my mentor and thesis chair, Ellen Skinner, for providing guidance and support with such clarity, authenticity, caring, and commitment. I am profoundly grateful to her for helping me get through the forest of this thesis process and, in doing so, helping me to really learn something about motivational resilience.

I would like to express my deep appreciation to my family for their loving, earnest, and integral support throughout this project. I owe a great deal to my colleagues and friends for providing emotional support, parallel play, and much-appreciated expertise and assistance in completing this project. Finally, I would like to thank the students, teachers, and administrators at Lane Middle School and the Learning Garden Laboratory for their dedication and for teaching us so much. 


\section{Table of Contents}

Chapter/Section Page

Abstract .........................................................................

List of Tables ...................................................................... vi

List of Figures.................................................................... vi

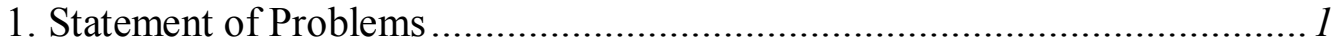

2. Review of the Literature on Engagement and Self-determination Theory..... 9

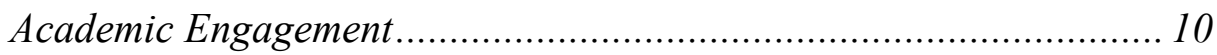

Self-determination Model of Engagement.................................. 16

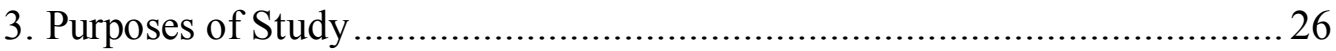

Motivational Resilience and Risk in Education.............................. 29

Cumulative Risk in Eighth Grade............................................... 31

Developmentally-calibrated Resources for Academic and Motivational

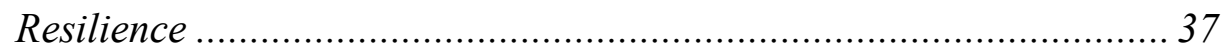

Research Questions and Hypotheses ......................................... 72

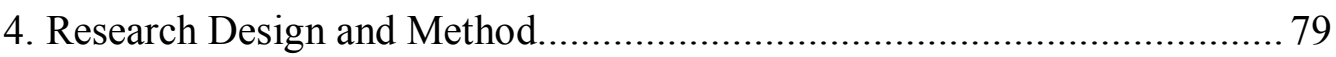

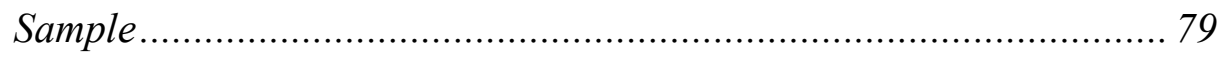

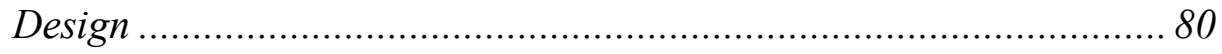

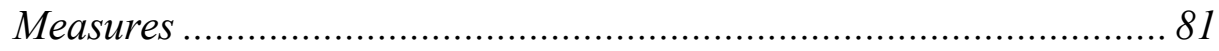

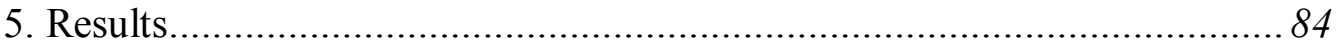

Preliminary Analyses ............................................................ 85

Research Question 1: "Eighth-gradeness” and Resilience Groups... 100 
Research Questions 2 - 4 ANOVAs: Resilience Group and

Developmental Resource Levels for Eighth-vs. Sixth-Grade Students 106

Research Questions 5: Enabling and Protective Factors Predicting

Dual Resilience Group Membership...................................... 121

6. Discussion .................................................................................... 139

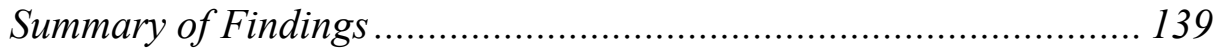

Strengths, Limitations and Future Studies.................................. 148

Implications ....................................................................... 154

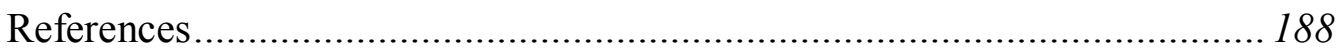

Appendix A. Measures ............................................................. 208

Appendix B. Supplementary Information: ANOVAS ............................ 211

Appendix C. Supplementary Information: Unique Effects ....................... 220

Appendix D. Supplementary Analyses: Mediations .............................. 222

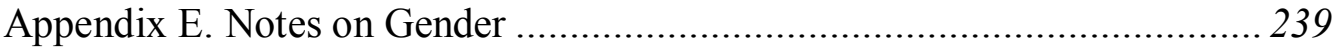




\section{List of Tables}

Table 3.1. Summary of research on autonomy predicting engagement and academic outcomes. 49

Table 3.2. Summary of research linking teacher support and engagement...... 55

Table 3.3. Overview of the Elements of the Learning Garden Laboratory......... 70

Table 5.1. Descriptive Statistics and Scale Properties .............................. 89

Table 5.2. Table Summary of Descriptive Statistics and Measurement Properties for Sixth- and Eighth-grade Students......................................... 91

Table 5.3. Inter-correlations among Study Constructs.............................. 94

Table 5.4. Inter-Correlations for Eighth-and Sixth-grade Students................ 96

Table 5.5. Mean Levels of Motivation and Achievement by Grade and Gender. 101

Table 5.6. Resilience Group Sizes and Range of Scores............................. 104

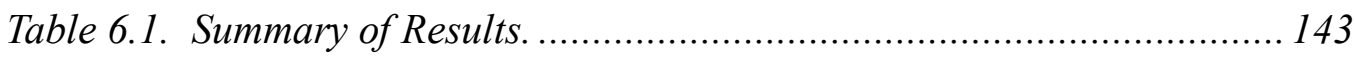




\section{List of Figures}

Figure 2.1. Self-System Model of Development.......................................... 17

Figure 3.1. Calibration of Self-System Motivational Model of Development:

Selected Processes within Schools Salient for Early Adolescents......... 42

Figure 5.1. Mean Levels of Autonomy, Teacher Support, Peer Support, and Garden Engagement in Eighth- and Sixth-grade Motivational Resilience

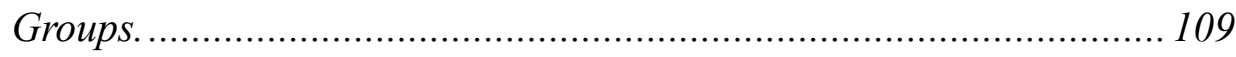

Figure 5.2. Mean Levels of Autonomy, Teacher Support, Peer Support, and Garden Engagement in Eighth-grade Academic Resilience Groups.... 113

Figure 5.3. Mean Levels of Autonomy, Teacher Support, Peer Support, and Garden Engagement in Eighth- and Sixth-grade Dual Resilience Groups.

Figure 5.4. Mutli-group Multinomial Logistic SEM: Grade Differences in Unique Effects of Developmental Resources on Dual Resilience Group Membership. 124

Figure 5.5. Mediated Logistic Regression: Autonomy as a Mediator between Eighth-Grade Dual Teacher Support and Dual Resilience Group...... 132

Figure 5.6. Mediated Logistic Regression: Autonomy as a Mediator between Eighth-Grade Garden Engagement and Dually Resilient Group........ 134

Figure 5.7. Mediated Logistic Regression: Autonomy as a Mediator between Sixth-Grade Teacher Support and Dual Resilience Group............... 136 
Figure 5.8. Mediated Logistic Regression: Autonomy as a Mediator between Sixth-Grade Garden Engagement and Dual Resilience Group........... 137 


\section{CHAPTER 1. \\ STATEMENT OF PROBLEMS}

Eighth grade seems to be a key potential turning point for students' academic success. Early adolescence brings myriad new capabilities just before the new social and academic contexts found in high school offer students a chance to reinvent their academic identities. This chance comes at a key time: Students' performance in high school may directly influence their college opportunities (National Center for Education Statistics, 1999) or career paths (Isakson \& Jarvis, 1999). At-risk students, in particular, may need exemplary performance and grades during high school to qualify for aid that can supplement the potentially prohibitive costs of college (NCES, 1999). These students, faced with additional difficulties, may need extraordinary motivation to navigate hurdles to success that are difficult for anybody, such as a changing job market (Côté \& Bynner, 2008) and an often onerous college application process (Horn, Chen, \& Adelman, 1998).

However, instead of developing the increasingly strong achievement and motivation that students need to shape their futures, the opposite trend is evident. Student's academic success and motivation tend to steadily decline as students age, with several periods of steeper declines, culminating in what can be an academicallyterminal drop over the transition to high school (J. S. Eccles, Lord, Roeser, Barber, \& Jozefowicz Hernandez, 1997). These general negative academic trends are more severe for ethnic minority and low-income students (Wigfield, Eccles, Schiefele, Roeser, \& 
Davis-Kean, 2006). Moreover, while a new context in high school could serve as a fresh start for disenfranchised middle school students, opportunities for malleability tend to dissipate quickly: Students who embark on a downward path in $9^{\text {th }}$ grade find it hard to recover (Roderick \& Camburn, 1999). Motivation seems to stabilize, for better or worse, after eighth grade (Gottfried \& Fleming, 2001; Marcoulides, Gottfried, Gottfried, \& Oliver, 2008; Pekrun, 1993). What would it take for at-risk students to complete eighth grade ready to succeed in high school?

By all measures, students in eighth grade should be increasingly equipped to do well. Early adolescents have new capabilities and interests that could help them perform better in school. Piaget's stages of cognitive development place eighth graders as just getting comfortable in formal operations (Inhelder \& Piaget, 1958); more able to exercise abstract, complex thought, and to assign and evaluate meaning in their surroundings (Fry, 1998). Students also have increased capacities for self-regulation and increased desires for self-direction and meaningful work (Damon, Menon, \& Bronk, 2003) that could help students take ownership of their school experiences as they prepare for college or a career. These same capacities could help students have more sophisticated interactions with teachers, actively participating in their own education and taking better advantage of support provided by teachers. Early adolescents desire more autonomy (J. S. Eccles et al., 1991) just as they are often expected to take more responsibility for managing their own schoolwork. They also place more importance on their peer relationships, potentially creating the type of 
support networks that should help students succeed in school (Wentzel, 2009).

Despite these emerging capabilities and characteristics, there are steep declines in motivation and achievement across adolescence (Lepper, Corpus, \& Iyengar, 2005). Just as school should be more relevant - directly informing students preparation for future college or career experiences - students rate school as being less useful and important ( Eccles, Wigfield, \& Schiefele, 1998; Wigfield, Eccles, \& Yoon, 1997). Among other motivational factors, students' levels of engagement (Skinner, ZimmerGembeck, Connell, Eccles, \& Wellborn, 1998) and intrinsic motivation (Gottfried, Fleming, \& Gottfried 2001) decrease during middle school, dropping sharply during the transition to high school (Wigfield, Eccles, Roeser, \& Schiefele, 2008). Academic performance and grades also tend to decline, both across middle school and at the transition to high school (Alspaugh, 1998). These decreases in motivation and achievement are associated with decreases in school retention (Janosz, Archambault, Morizot, \& Pagani, 2008) that have reached near-epidemic levels. Across the United States, only two thirds of students graduate high school in four years, on average, and only about half of urban, low-income, or ethnic minority students do so (Greene \& Winters, 2006). While absenteeism, course failure, and other early warning indicators can identify students who may drop out (Roderick \& Camburn, 1999) there is much less known about predicting students' academic resilience.

Indeed, understanding student failure is a complementary but separate undertaking from understanding how students can succeed in middle school in order to 
be ready for high school. Success and readiness require specific skills, perspectives, and motivational resources that are not captured by the simple absence of risk factors. Positive Youth Development (PYD) has, in part, taken up the call to focus the spotlight on students' strengths, and away from deficits. Specifically, PYD “emphasizes the manifest potentialities" of all young people, including those "from the most disadvantaged backgrounds and those with the most troubled histories" (Damon, 2004, p. 2). Indeed, as discussed above, the eighth grade experience seems to be rife with "potentialities." What is lacking is an understanding of exactly what those developmental opportunities entail and how schools can support them. Some PYD theorists have called for a stronger developmental perspective within the field (Larson, 2000), but even within motivational theories rooted in the study of developing students, developmental attunement to the specific "potentialities" of eighth graders is generally absent. Which aspects of academic success are particular to students in this age group? Stage-environment fit theory (Eccles et al., 1993) has explored the motivational needs that are particular to early adolescence, and ways in which schools can meet (or fail to meet) those needs. Evidence from a large body of research on stage-environment fit suggests the lack of fit between adolescents' developmental needs and their academic environments is a major reason for the academic declines seen across adolescence (Eccles et al., 1996). At the same time, many of the theories used to study adolescent motivation (for example, the needs for autonomy, competence, and relatedness posed by self-determination theory; Deci \& Ryan, 1985) are considered useful in part because 
they are seen as universal and supported empirically regardless of subjects' ages (e.g., Grolnick, Gurland, Jacob, \& Decourcey, 2002). However, considering the radical developmental changes that students undergo during their academic careers (Anderman \& Maehr, 1994), developmental attunement to the particular motivational needs of eighth graders may be necessary to support transitioning students' success and readiness. If, schools were able to address the particular developmental needs of eighth graders, harnessing their developmental strengths, the result might be a motivational pattern that meets the burgeoning promise of that age group.

Thus, this project will examine the developmental opportunities that seem to emerge during middle school. Students in eighth grade should have increasing capacities for and prioritization of academic ownership and self-direction, commitment to purposeful and authentic tasks, collaboration with peers, and sophisticated interactions with teachers. Accordingly, when considering the aspects of students' academic experiences that strengthen motivation, this study will investigate four factors that should be particularly salient for eighth-grade students: students' feelings of autonomy in school, engagement in purposeful and authentic academic work, perceptions of supportive peer relationships, and perceptions of attuned motivational support from teachers. If the interactions between students and their academic environments do not foster these factors and students do not feel autonomous, meaningfully engaged, and supported by peers and teachers, these unmet developmental priorities would become liabilities -- as seems to be the unfortunate norm. As explored 
in stage-environment fit research, the mismatch between students' development and schools seems to be a major contributor to widespread processes of increasing disaffection as students age. To the extent that these priorities can be actualized in school, a fulfillment of students' capacities may offer a route to motivational resilience and, consequently, achievement for at-risk students.

In this study, students' emotional and behavioral engagement with their schoolwork are of special focus as key markers of motivation and core ingredients of school success for at-risk eighth-grade students. The extent to which students actively participate with and are energized by their work (Skinner, Kindermann, Connell, \& Wellborn, 2009) is especially important when considering the importance of identify formation in early adolescence. The search for avenues in which to be successful, be one's self, and belong is a key part of adolescence (Roeser et al., 2008). If students are not engaged in school, they may choose other avenues for engagement and divert their efforts accordingly.

Conversely, engagement seems to be a key pathway to school success for those who are at-risk for negative academic outcomes. Even though poverty and a marginalized ethnic group status are associated with lower achievement (Wigfield et al., 2006), increases in engagement can predict increases in achievement better than ethnicity or income (Fredricks, Blumenfeld, \& Paris, 2004). Students' engagement is a strong and reliable predictor of academic achievement (see Wigfield, Eccles, Roeser, \& Schiefele, 2008), but unlike demographics or other fixed predictors, engagement is 
malleable (Fredricks et al., 2004). Understanding how eighth-grade students' motivation is fostered, and specifically how students maintain high levels of motivation despite the declines predicted both by demographic risk factors and by simply becoming older, may help unlock a route to students' academic success.

Several motivational models offer an explanation for how students' motivation can be fostered, often emphasizing student's beliefs about their capabilities and identities see (see Wigfield et al, 2006). Self-determination theory (SDT; (Deci \& Ryan, $1985,2000)$ and the self-system model of motivational development (Connell \& Wellborn, 1991) offer particular utility in explaining students' motivation. By supporting students' intrinsic needs for autonomy, competence, and relatedness, teachers, peers, and other partners that compose students' contexts can support students' self-perceptions as being autonomous, competent, and belonging. This contextual support and corresponding change in self-perceptions foster students' engagement or, when not supported, disaffection, which both contribute to students' achievement in school and feed back to inform future interactions with contextual partners and selfperceptions. SDT and the self-system model has been helpful in understanding and optimizing engagement in school settings, demonstrating that there are malleable factors that can be used as pathways for improving classroom environments and reengaging disenfranchised youth (Ryan \& Deci, 2009).

By using stage-environment fit to select aspects of SDT and the self-system model that are salient to early adolescents, this study seeks to both form an 
understanding of motivational resilience in this age group and to incorporate the agegraded calibration highlighted by stage-environment fit to the widely used SDT model. Thus, this project will identify at-risk eighth-grade students who are both motivationally resilient (high in engagement) and academically resilient (high in achievement), and examine whether their ratings of teacher support, peer support, autonomy, and engagement in a hands-on, project based school garden can predict differentiated resilience versus non-resilience in these students. 
Chapter 2: Review of the Literature on Engagement, Resilience, and SDT

\section{CHAPTER 2.}

\section{REVIEW OF THE LITERATURE ON ENGAGEMENT AND SELF-DETERMINATION THEORY.}

Given the need to understand how to protect and foster early adolescents' academic success in the face of demographic risk factors and normative age-graded declines, a motivational framework was chosen to facilitate this understanding. Work on resilience, including a modest body of work on academic resilience, enables the exploration of how certain motivational factors can buffer or enable success in the face of risk factors. Stage-environment fit theory raises the importance of meeting adolescents' developmental needs in order to enable them to develop the healthy selfbeliefs and motivation that they need to succeed in school. Self-determination theory and the self-system model of motivational development (SSMMD) offer engagement as a key factor in academic success in conjunction with several of the motivational processes that seem to be central for both protecting and enabling adolescents' motivational success.

Because work on engagement has burgeoned in recent years, an overview of research in that area will be provided first, followed by an explanation of its distinction and use in self-determination theory perspectives on education. The final sections in this chapter consider how self-determination theory can be expanded and brought more in line with the idea of stage-environment fit by incorporating aspects of work risk and resilience. 


\section{Academic Engagement}

The construct of academic engagement has become a rallying point in educational research because of its utility in facilitating understanding and improvements in students' school lives. Not only is engagement notable as a strong predictor of important educational outcomes that help explain students' educational development, it also seems to capture a malleable "active ingredient" in students' education that goes beyond demographic risk factors. Although ideas vary as to what is and is not included in the construct of engagement (see Fredricks, Blumenfeld, \& Paris, 2004; Skinner, Kindermann, Connell, \& Wellborn, 2009 for reviews), these diverse conceptualizations converge in a few powerful aspects.

Engagement, at its most basic, describes what happens in the point where a student meets her classroom environment. It captures both what the student thinks and feels about work in the classroom and what she does in class, incorporating cognitions, emotions, and behaviors (Jimerson, Campos, \& Greif, 2003). For an engaged student, those interactions with the class activities are energized, focused, active, and sustained (Skinner, Kindermann, \& Furrer, 2008), encapsulating not only a student's work in the classroom but her "quality of participation with academic activities" (Skinner et al., 2009 , p. 227). The study of engagement, thus, allows a glimpse into the significance student actions take in the classroom, examining the aspects of those actions that are important to the student and her teacher.

Disaffection is a conceptualization of what happens when students lack 
engagement. That is, if engagement is the quality of participation in the classroom, disaffection addresses not only low levels of participation but all of the ways that students withdraw participation. Disaffected students, then, may be bored, inattentive, or doing the bare minimum, but they might also be actively disruptive, off-task, or apathetic (Skinner et al., 2008).

Conceptualizations of engagement and disaffection can be organized, roughly, by their focus on student's emotional, behavioral, or cognitive interactions with school and schoolwork. Some framings of engagement focus on one aspect; for example, school bonding is among the framings of engagement that focus on students' emotional connection to school or schoolwork (e.g., Libbey, 2004). However, all conceptualizations of engagement include at least two core components: behavioral and emotional engagement.

\section{Components of Engagement}

Behavioral engagement describes students' outward participation in academic activities, including both compliance with expectations and active work on academic tasks (see Fredricks et al., 2004). It is this behavioral aspect that others can most easily observe, and that leads to outcomes such as test scores and grades. Emotional engagement, on the other hand, addresses students' internal states such as their feelings of energy or enjoyment, reactions to teachers and other contextual partners, their interest in a subject, and feelings of connection with school.

Each of these components may serve a different role in student's academic 
success and development. For example, Skinner and Belmont (1993) found that perceptions of teacher support were more strongly related to behavioral engagement than emotional engagement, whereas Furrer and Skinner (2003) found that perceptions of peer support were more strongly related to students' ratings of emotional engagement than behavioral engagement. When looking within an individual, the components of engagement can be conceptualized as separate steps in a motivational process. For example, in Finn and colleagues' participation-identification model (Finn \& Zimmer, 2012), in which behavioral engagement leads to academic outcomes, which in turn foster a student's identification with school (emotional engagement), further supporting behavioral engagement. After following students from eighth grade to graduation, they found that found that students' behavioral engagement mediated a positive relationship between emotional engagement and dropout. However, when considering a student's entire system, it can be useful to think of the point at which these differing components intersect.

It is often the combination of behavior and emotion into a cohesive action that encapsulates the impact a student's action may have. By considering all aspects of a student's engagement concurrently, one is sometimes able to paint a more relevant picture of a student's engagement. For example, regardless of a student's emotional engagement, a student may not learn if they do not apply sufficient effort behaviorally. This is not to say that the student's emotion does not drive her behavior, but rather that, when considering the mechanisms that both influence and are influenced in the 
student's environment, it can be useful to think of engagement as an action incorporating all aspects of engagement.

\section{Importance of Engagement for Academic Success}

Engagement has gained such popularity among educators because it supports academic success in multiple ways. Most notably, it has strong empirical ties to important academic outcomes such as achievement and dropout (Fredricks et al., 2004).

Engagement is related to important outcomes. Given that engagement and disaffection capture the ways that students interact with academic material, it may be no surprise that students' engagement is a strong predictor of their academic success. Research has consistently shown positive links between engagement and achievementrelated outcomes such as grades and test scores, as well as with attendance, school completion, and retention (e.g. Connell \& Wellborn, 1991; Marks, 2011; Skinner, Wellborn, \& Connell, 1990), for a review, see Fredricks et al., 2004. For example, one study in three independent, highly diverse samples of adolescents found that engagement was related to attendance, test scores, and achievement tests (Connell, Spencer, \& Aber, 1994).

Moreover, engagement is related to these positive outcomes above and beyond both status indicators and previous achievement. For example, Finn and Zimmer (2012) found that those with higher engagement in eighth grade had markedly better odds of avoiding dropout, even after controlling for prior achievement, school variables such as size and being an inner-city school, and student variables such as gender, ethnicity, and 
receiving free lunch. That is, it does not seem to be the case that engagement is simply a proxy for overall school success as dictated by, for example, student's academic aptitude or income levels. Rather, one interpretation of these data is that engagement captures a route to academic success that can be used by any student.

Engagement is an important pathway to academic success. Some researchers propose that engagement is not only related to learning, it is the means by which students' learning and academic development occurs (Skinner et al., 2009). Students' interactions with teachers and academic materials can only impact students if those students apply their attention, effort, energy, or own goals to those interactions. Additionally, the processes involved in engagement can be seen as important academic skills and desired outcomes themselves. The ability to persist in the face of difficulties and self-regulate (Finn \& Zimmer, 2012) is vital for students to succeed in and out of school. Coming up with new ideas and thinking beyond the scope of what is required may help students' academic progress, and some suggest that non-traditional cognitive skills similar to these may be especially important given then increasingly global and changing nature of our workforce and culture (e.g. Roeser \& Peck, 2009).

Engagement is both enduring and malleable. The benefits of engagement are especially powerful because, unlike demographic variables, engagement is malleable. Because engagement is a process, it can be directly shaped by the actions of both the student and his social partners. When interventions help one aspect of the student's school experience be more motivationally supportive, students engagement increases, 
whether that aspect be schools (Finn \& Voelk1, 1993; Newmann, Wehlage, \& Lamborn, 1992), teachers (Reeve, 2006), or students' own perceptions of themselves (Blackwell, Trzesniewski, \& Dweck, 2007).

At the same time, the effects of engagement are long-lasting. Several researchers have found that engagement in elementary or middle school predicts engagement and achievement years later (Finn \& Zimmer, 2012; Fredricks et al., 2004). For students with low engagement and those with demographic risk factors, this stability can be problematic: Students who are doing poorly tend to keep doing poorly, just as students who do well tend to keep doing well (see Skinner et al., 2009).Thus, engagement is something that can be changed, but is not transitory, suggesting that a focus on supporting engagement can be an especially powerful tool for promoting changes that carry forward in students' lives.

In part, perhaps, because it is both malleable and self-sustaining, engagement is especially important for at-risk students (Finn \& Zimmer, 2012). Not only is engagement a strong and consistent predictor of positive outcomes in samples of at-risk students (see Fredricks et al., 2004), it also seems to be related to processes that are particular to the experiences to at-risk students. A sense of belonging and acceptance at school can be a major barrier to students' academic success (Walton \& Cohen, 2011) and engagement is closely tied to aspects of school belonging. Issues of identity can raise particular problems for adolescents in marginalized groups when students face a perceived choice between embracing a negatively-stereotyped cultural identity or 
sacrificing an aspect of one's identity in order to be successful (McInerney, Roche, \& Marsh, 1997; Oyserman \& Bybee, 2006). Student's engagement can be considered both as one of the means by which these conflicting identity issues emerge and as a potential route through which students defy negative trends to succeed.

\section{Self-determination Model of Engagement}

As portrayed in the burgeoning research, engagement is such a complex concept that it can be hard to articulate the exact meaning of concepts related to its study. Where does engagement stop and the consequences of engagement begin? To what extent are students' emotions or cognitions about themselves a cause of engagement as opposed to being an aspect of engagement itself? The self-system model of motivational development (SSMMD; Wellborn \& Connell 1991) organizes the precursors and outcomes of engagement. The SSMMD examines the motivational support offered by students' contextual partners, describes how that contextual support influences students' self-perceptions, and shapes the students' resulting actions in the classroom, and the outcomes those actions create. Thus, the SSMMD has been useful because it groups social factors and personal factors that promote engagement and connects those factors to academic consequences.

In this model (see Figure 2.1), engagement and disaffection with academic tasks are viewed as the action a person takes, i.e. the observable manifestation of internal motivation. Leading up to this action, the SSMMD considers the context of the person; that is, the people with whom a student interacts, and how those interaction partners 
support his motivation. The student, in turn, perceives these interactions between himself and interaction partners, making appraisals of his own self in relation to his context. These perceptions of the students' interaction with his system -- self-system perceptions or SSPs - promote or undermine student's own naturally-occurring

Figure 2.1. Self-System Model of Development (Connell \& Wellborn, 1991). Dashed aspects of the model are more recent additions (Skinner \& Pitzer, 2012.)

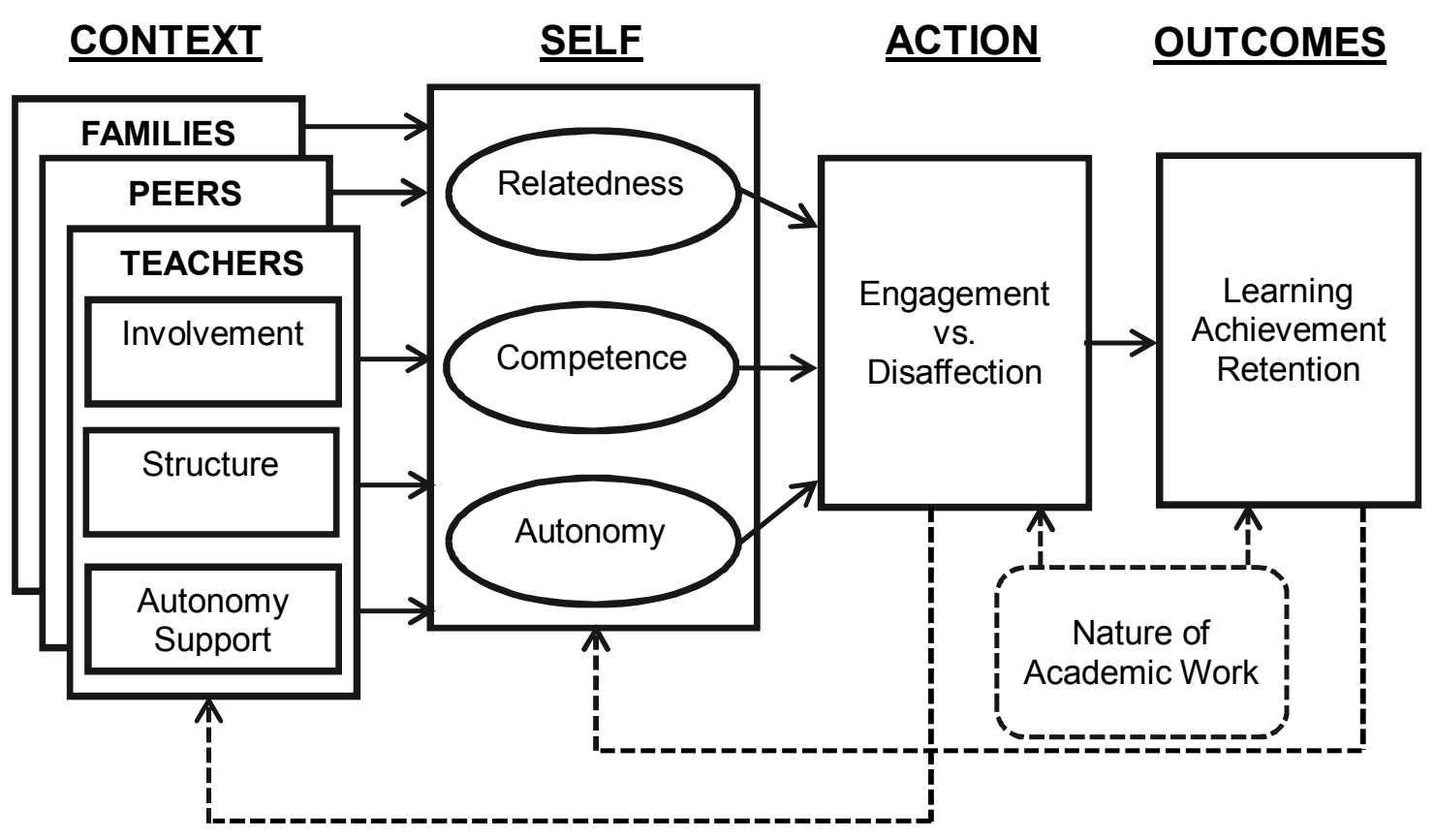

motivation, from which springs his or her engagement with academic activities. 
Through this engagement (or, as it may be, disaffection) with academic work, the student learns and demonstrates mastery. This engagement or disaffection also shapes the student's own perception of himself, as well as giving teachers information on the student's progress. In this way, engagement shapes learning, achievement, school retention and, in recent renditions of the model, relationships with teachers. Considering engagement as an action within a larger context-self-action-outcome model also facilitates the consideration of engagement as an interactive process - one that is also shaped by the nature of the academic work that students are asked to perform. In this model, the cultivation of motivation is informed by self-determination theory (Deci \& Ryan 1985), which holds that students have universal needs for relatedness, competence, and autonomy. For all students, it is important to feel liked and accepted, to feel capable and efficacious, and to feel the freedom to express one's true self. The people and situations in a student's environment can support these needs, allowing the student to perceive him or herself as related, competent, and autonomous. These feelings support the students' own intrinsic motivation and help the student be more engaged in school.

Autonomy and autonomy support. Supporting autonomy is a particular challenge in schools. Perceived autonomy in schools is the freedom to express one's true thoughts and feelings, the ability to both act upon one's own volitions and to be authentic to one's inner self. Traditionally, schools necessitate a certain level of conformity and control for a classroom to run smoothly, but teachers can support the 
need for autonomy by giving students choices, by providing rationales that explain why tasks are important and personally relevant to students, and by listening to students' input and taking their voices seriously. In this way, students can feel a sense of ownership and purpose in their education.

Competence and structure. School environments are generally more conducive to some of these needs than others. The idea of competence is central in school environments. Competence draws on previous investigations of self-efficacy, locus of control, learned helplessness, and perceived control, encompassing a broad picture of a student's beliefs about his or her ability to be effective. Students who feel competent know what to do, how to do it, and are confident that they are able to complete those steps. They feel capable and expect to succeed. Teachers and others in a student's environment support competence beliefs by providing good structures: clear instructions, high expectations, good information about the means to complete the tasks, and rich feedback about performance so that students walk away with knowledge, skills, and confidence.

Relatedness and involvement. While teachers are familiar with the importance of relationships with students, schools can be a difficult place in which to support students' perceptions of relatedness. Building on research on attachment and pedagogical caring, the SSMMD posits that a student with a strong sense of relatedness feels that he is liked by teachers and peers, that he fits in and belongs at the school, and that the school is a good place for people like him. Students with a high sense of 
relatedness believe that they are likeable and loveable, and that they will be accepted. Teachers can support this belief by expressing warmth and care, by being available and dedicating time to students, and by getting to know students and building individual relationships so that students feel secure and valued.

Students' experiences of teacher context. Moreover, students' needs are generally not met in isolation. That is, intuitively, it may seem that a teacher would have to choose which need to support. Perhaps the guidelines that supporting competence would cancel out the freedom that support autonomy, or that giving students space to support their autonomy would not mesh with the closeness that comes with supporting relatedness. However, some researchers have begun to detail how all three needs support one another (Jang, Reeve, \& Deci, 2010). As they discuss, are not posed with a choice between providing autonomy support and providing good structures. Rather, an environment that truly supports autonomy must also support competence and relatedness. For example, if teachers provide high expectations and meticulous instructions (supporting competence) but students do not see any value to the work, sense that it is relevant, or feel that they have options to tailor the work so it feels important (autonomy), students may feel less competent than before. On the other hand, when teachers give students a clear map that shows how to accomplish something both difficult and personally useful to students, with strategies for overcoming difficulties and ways for students to guide their own learning, students can feel more capable (i.e. feel competent) and truly identify with the work they are doing (i.e. feel 
autonomous).

It is important to note that the experiences of a presence or absence of involvement, structure, and autonomy support are subjective. A teacher may believe he is supporting his students' autonomy by giving them free reign over an activity, but the students may perceive a lack of guidelines as a sign that the task is not important, and having to do an unimportant task corresponds with a perceived lack of autonomy. Alternately, a student in a formal, high-performance environment where there is little outward expression of affection may still feel a high sense of belonging because she feels like an important and contributing member a prestigious system.

At the same time, when a teacher consistently supports or does not support a need, there can be a predictable result for a student. If students receive consistent feedback from their environments that contradicts their motivational needs, it can be almost impossible for students to maintain a belief to the contrary. For example, if a student has the constant experience that, no matter how hard he studies, even if he thinks he's studying the right material and practicing in a way that should prepare him for the exam, he fails exams in a course, it will be very difficult for him to feel competent in that course. More likely, he will believe that he doesn't have what it takes to succeed in that subject. The processes of feeling autonomous or engaged live neither in a student's environment nor solely in her psyche, but as an ongoing interaction between the two.

\section{Authentic Learning Experiences}


While focusing on the important role students' contexts play in supporting or undermining their engagement, it can be easy to lose track of the actual work with which students must engage. At some point, even the most supportive teaching and highest levels of engagement with academic tasks cannot produce learning outcomes if the academic work itself is of low quality. In order for students to learn and achieve, academic tasks must be well-designed, content-rich, and appropriate for students.

Moreover, some research has begun to examine the way that the nature of academic tasks can support motivation (Wigfield, et al., 2006). To promote development along with academic competence, academic tasks benefit from being authentic and purposeful. Contextual learning, that is, learning that is hands-on, project-based, and rooted in real-world activities, may facilitate motivationally supportive student-teacher interactions and the feelings of commitment and interest that help make up the core of engagement.

\section{Engagement as an Action}

Considering engagement as a reaction to motivationally supportive teaching and authentic academic tasks - or the lack thereof - allows a deeper understanding of engagement itself. In this framing, the concept of reaction is literal: an action in response. But what is meant by an action in this model? Action theories (see Skinner et al., 2009 for a summary) view a unit of action as one that is goal-directed, including behavior, emotion, and attention. Consideration of engagement as an action helps to explain the way behavior and emotion together shape a student's academic experience. 
In order to truly understand how a student's action impacts himself and his teachers, one must consider that student's behavior and emotion concurrently. For example, for a high-achieving student, simply completing an assigned reading (high emotional engagement) may be an action that's he feels is boring (low emotional engagement) and not helpful to his learning, and is equally unimpressive for his teacher. On the other hand, for a failing student, completing the same reading may serve as a milestone, demonstrating that the student can be successful in the classroom, thus energizing the student (high emotional engagement) and showing a teacher that the student cares about the class. The same behavior, completing a reading assignment, can be a wildly different "action" depending on the emotion and cognition that a student brings to the situation.

The concept of an action also helps explain why engagement is such a powerful construct. One of the reasons that traditional markers of academic risk such as discipline issues and absences (Roderick \& Camburn, 1999) is that they tell a story about a student: He is rebelling against school, or she has checked out. In addition to the pragmatic benefits of staying out of fights or attending classes, it is the action of this story - has she stopped caring about school, or was she ill? - that concerns administrators, and engagement is in some way the root of that underlying story. Thus, contemplating the "action" segment of the model both helps illustrate why it can be useful to use behavioral and emotional measures in conjunction, and why it is that engagement should be such a powerful predictor of academic outcomes. 


\section{Reciprocal Effects}

Not only does the action engagement "feed-forward" into learning and achievement outcomes, it also serves as feedback to teachers or other partners who then respond in kind to students (Skinner et al., 2009). While less-studied than many other facets of engagement, these reciprocal processes help explain patterns in which highperforming and low-performing students tend to stay that way. Teachers tend to respond more positively to engaged students, at times being more motivationally supportive. In turn, this helps shapes students' feelings of autonomy, competence, and belonging in school, which promote engagement and restart the cycle (Skinner \& Belmont, 1993). The opposite cycle is seen with disaffected students, who can elicit teacher frustration or withdrawal, promoting further disaffection and reduction in learning.

Within populations whose experiences may be disadvantaged by, for example, poverty or discrimination, their levels of engagement or teacher support may start out low. Left uninterrupted, the reciprocal effects of engagement tend to cement trajectories in which the motivationally "rich" get richer, while the motivationally "poor" only decline further (Skinner et al., 2008). Self-reinforcing processes in the SSMMD of engagement seem to be one mechanism through which income level, ethnicity, and other demographic risk factors hurt students' progress.

At the same time, the processes involved in engagement offer one answer to improving these students' academic experiences. Unlike the underlying factors 
involved in poverty or other risky situations, engagement is relatively malleable in the classroom. The SSMMD gives routes by which even previously disaffected students can step into self-reinforcing interaction patterns that lead to academic success (Van Ryzin, 2011. Even when not directly measured, feedback loops within the SSMMD help explain why the model should predict differences in students' trajectories over time. Thus, it can be helpful to consider that any piece of the model that is being measured may be a part of a larger self-sustaining process of betterment or decline. The self-system model of motivational development illustrates a system that is dynamic (see Figure 2.1). A student's context influences his self-perception, which then informs his action of engagement or disaffection in regard to academic work that is meaningful or not, giving rise to academic outcomes such as learning and achievement, which then help begin the cycle again. 


\section{CHAPTER 3.}

\section{PURPOSES OF STUDY}

In research involving students with demographic risk factors, studies abound on students' low achievement, disaffection, and drop-out. Much is documented about what goes wrong for at-risk students, but despite enthusiasm for the study of positive youth development (Damon, 2004), much less is understood about what can go right in schools for these students. For low-income, ethnic minority, and immigrant adolescents, positive educational outcomes may be even more important as a route to success, yet such outcomes are at lower levels than for other young people. Many schools use checklists of risk factors that effectively identify students who are in danger of school failure or dropout. An understanding of what can go wrong in schools is, of course, a helpful step in identifying the trajectories of students who are off-track. This focus, however, is divorced from an understanding of what can go right within populations of students who traditionally do less well in school (Connell, Spencer, \& Aber, 1994).

Research focusing on resilience investigates processes that lead to success despite risk. Growing out of the more traditional research on risk factors and their associated problems (Luthar, 2006), resilience research allows an understanding of those who are at-risk, but in some way buck the expected negative outcomes. This research seeks to understand protective factors that may buffer the negative effects of risk factors as well as to locate enabling factors that might even promote thriving 
despite high risk (Bandura, 2006; Martin \& Marsh, 2009). A focus on protective and enabling factors allows a proactive, rather than reactive, approach that can allow schools to foster students' success before students experience problems that may be difficult to reverse.

The goals of the current study are to examine the personal and interpersonal predictors of motivational and academic success in young at-risk adolescents, using frameworks supplied by work on self-determination theory, resilience and selfdetermination theory. In order to adequately capture the specific motivational resilience of early adolescents, it combines these theories to explore the developmental calibration of SDT and resilience frameworks. By outlining general processes of resilience, populating those processes with motivationally important factors from SDT as identified by stage-environment fit, and then taking a developmental perspective on those processes and factors, this study intends to contribute to an understanding of how demographically at-risk eighth-grade students can be academically and motivationally resilient.

Resilience work, via the investigation of risk factors, positive adaptation to risk, and protective or enabling factors, provides a way to understand the cultivation of successful outcomes in problematic situations. Stage-environment fit likewise considers how to capitalize on sensitive situations. In particular, stage-environment explores how the same developmental needs can, depending on the fit provided by environment, either serve as resources or liabilities for students (Eccles \& Midgley, 1989). Self- 
determination theory, on the other hand, offers a robust understanding of factors key for positive educational outcomes in all educational environments, and thus can offer candidate constructs for further exploring enabling or protective factors as proposed by both resilience and stage-environment fit. Additionally, SDT offers engagement as a valuable core indicator of motivational resilience, accompanying the traditional use of achievement as a standard by which to measure positive adaptation. The contextual and personal factors depicted in SDT and stage-environment fit can support an individual's capacity to fulfill her potential, and thus should function as enabling factors for success in the face of risk.

Although stage-environment fit research has demonstrated the importance of considering students' particular developmental needs, education research within both the resilience and SDT approaches tends to lack any explicit consideration of developmental changes. This lack of developmental calibration is problematic when considering the developmentally-timed nature of educational declines. Hence, a major goal of this study is to explore the developmental calibration of resilience and SDT for at-risk eighth-graders. A developmental perspective taken at the intersection of these three frameworks allows 1) a consideration of what positive academic and motivational adaptation would look like in consideration of combined risks posed by both being in a traditional sixth-to-eighth-grade middle school and in demographic risk groups, 2) the consideration of "eighth-grader-ness" itself as an additional risk factor in this profile of cumulative risk and finally 3 ) the consideration of which enabling factors might foster 
these kinds of resilience at this particular stage in students' academic development. The following section considers each of these issues in turn.

\section{Motivational Resilience and Risk in Education}

In order to identify risky and protective factors for academic resilience in children, we must first know what resilience looks like. Rather than being a static or trait-based label, resilience is a process, an ongoing positive adaptation to a risky situation, and, ideally resilience research identifies those adaptive processes themselves (Luthar, 2006). Measurement and observation of these processes, however, is problematic. Far more often, researchers explore the relationships between risk factors and positive adaptation as an approximation of measuring actual resilience. Once risk factors are identified, people following a resilient trajectory are those who fare "substantially better than what would be expected given exposure to the risk circumstance being studied" (Luthar et al, 2003, p. 515). Luthar and colleagues suggest that resilience indicators should be both developmentally appropriate (i.e., something that would logically show positive adaptation given at a particular age) and conceptually linked to the risk processes (p. 515), as well as entailing something that makes a difference to future development.

\section{Engagement as a Marker of Motivational Resilience in Education}

Given these requirements, academic engagement offers particular promise as a marker of resilient academic development. Academic achievement, although it is one of the most salient markers of academic success, can be far removed from students' 
subjective experiences of school. More specific subjective constructs, such as academic identification, intrinsic motivation, and perceived competence, are deeply tied to the situational processes that put students at risk but often lack a behavioral component that reflects how well students are actually coping in school. Additionally, these conceptions seem to take on a different salience and meaning for students of different ages. A conceptualization of engagement involving both a subjective, emotional component and a concrete, behavioral component allows connections to the perceived experiences of students as well as to their more objective successful academic adaptation in a way that has a similar meaning for students whether they are sixth graders, eighth graders, or of any age.

However, very little research examines engagement as a marker of resilience. In school contexts, resilience has been explored via educational resilience, academic resilience, and academic buoyancy. These tend to focus on achievement, which is often the most important factor in policy and funding discussions. Also considered are socioemotional and mental health outcomes that are, suitably, tied to students' larger success. In these studies, engagement is conceptualized as a mitigating factor that can support other outcomes.

However, the few studies that have examined engagement as a marker of resilience suggest that it offers utility beyond that provided by other conceptualizations of resilience in school. In Klem and Connell's (2004) study, for example, identifying young people with resilient engagement offers teachers and schools a goal that is 
directly tied to students' experiences and heavily influenced by teachers. It seems clear that additional work focusing on engagement as a marker of motivational resilience could be helpful if schools are to support the developmental needs of all students.

\section{Cumulative Risk in Eighth Grade}

Risk factors are any conditions in children's lives, whether demographic, environmental, social, psychological, or behavioral, that increase the probability of negative (or decrease the probability of positive) adaptation and development. If motivation and achievement are key educational outcomes, which factors put students at risk for motivational losses and underachievement? As previously discussed, immigrant students and students who speak English as a second language, students who are members of ethnic minority groups, and students from low-socioeconomic backgrounds are among groups at risk for school failure and dropout. These same students experience gaps in motivation that are present when students begin attending school, and widen as they continue.

Some of the times of most heightened vulnerability, during which the gaps in demographically at-risk students' motivation tend to widen, are during school transitions. A large body of work focuses on the transition to middle school as a critical period of vulnerability for students' motivation. Students generally tend to experience drops in their intrinsic motivation, interest, academic engagement, and valuing of school, and all of these commonly observed losses are more severe for groups of students low in socio-economic status, who belong to ethnic minorities, who are from 
immigrant families or speak English as a second language, or otherwise belong to marginalized groups (Wigfield et al., 2006).

\section{Stage-environment Fit}

A key developmental explanation for motivational declines during the transitions to middle and high school posits as critical a widespread lack of fit between adolescent students' developing needs and the characteristics of their schools. As Eccles and her colleagues demonstrate through the concept of stage-environment fit, students' motivation depends on an environment that is suited to their developmental needs. During early adolescence, students more deeply take in feedback from their teachers, begin differentiating ability from effort, compare themselves more to their peers, and prioritize relationships with peers (Wigfield et al., 2008). These processes help shape each student's identity and contribute to decisions about the type of person they want to become (Roeser, Eccles, \& Sameroff, 2000). At the same time, however, as students move from elementary to middle school, and again to high school, schools become larger, less personal, more bureaucratic, more controlling, more evaluative and competitive, and more focused on grades and performance.

Thus, school transitions seem to present a risk factor to the extent that they create a poor fit between students' developmental needs and school environments. By considering these periods of vulnerability as risk factors in conjunction with demographic variables, researchers can consider what would promote resilience across periods in which positive adaptation can be very difficult. When schools adjust to better 
accommodate students' developmental needs, traditional declines in engagement can disappear (Eccles et al., 1996).

\section{“Eighth-gradeness" as a Motivational Risk Factor}

In combination with traditional status-based risk factors, it may be useful to contemplate the possibility that developmental status itself in a context of traditional middle schools' structure may be a meaningful risk factor. Developmental changes tend to bring added capacities, and adolescence is full of developmental gains. In school, however, the overall experience of growing older in general, and the particular experience of entering early adolescence, bring developmental advances that can also serve as liabilities for motivational and academic success.

For all young people, regardless of group membership, the myriad changes that come with early adolescence change the way students experience school (Anderman \& Maehr, 1994; Roeser et al., 2000). Rapid brain development, physical growth, changes in the endocrine system, and a shifting social atmosphere combine to elicit developments in abstract reasoning, identity formation, and renegotiation of social relationships. Students are developing interests outside of schools, often making their schoolwork less of a priority. At the same time that students are striving to form their own identities, they begin to see ability as a fixed trait that cannot be changed (Eccles \& Midgley, 1989).

Students at this age use feedback from their environments and their own emotions to shape their views of themselves, and that feedback often does not promote 
a connection with school. As students examine their identities and consider their future selves (Oyserman \& Bybee, 2006 they may concentrate on interests outside of school, or choose to disidentify with school if they are not already doing well, as a way to protect their identity. With a heightened focus on peer interests, students with academically engaged and successful peers may see themselves as lacking, and students with academically unsuccessful peers may seek to appear less academically focused.

\section{Confluence of adolescence, school structure and demographic risk factors.}

For students from groups that traditionally do less well in school, these same processes can take on a heightened meaning. Students whose groups do less well in school may internalize low expectations when forming their own ability beliefs (Blackwell et al., 2007). During identify formation, they may perceive a lack of academically and economically successful opportunities in life and thus begin to identify with role models on less-adaptive paths. As peers become more important, students may have increased doubts about whether they belong in school, or feel forced to choose between academic success and relationships with stereotypically poor-performing same-ethnicity peers.

In risk and resilience research, much risk is explained by cumulative risk factors (Masten, 2001): rather than any single risk factor, or the additive effects of several risk factors, real harm can come at the intersection of multiple factors in combination. For adolescents, traditional demographic risk factors seem to be of biggest concern when combined with a lesser-explored risk factor that all students experience - simply growing older. For students in demographically at-risk groups, motivational trajectories 
can hit dead-ends when societal disadvantage combines with the multiple changes of adolescence. Research on stage-environment fit suggests that these issues can be amplified or ameliorated by the structural changes in schools that transition to from elementary to middle school.

Together, these changes seem to catalyze what can be, for many, the final integration of one's view of oneself as a student. Students' beliefs about themselves as students seem to stabilize after this time (Eccles et al 1989, Wigfield et al 1997), and perhaps this contributes to a stabilization of motivation during adolescence. Pekrun (1993) and Gottfried and colleagues (2001) both found that after eighth grade or age 13, student's intrinsic motivation levels off - for better or worse.

The developments that come with eighth grade, then, could make it a critical period, a sort of "last chance" when motivation is in danger but--if new developmental capacities are put it use_-still perhaps salvageable. Eighth-grade students have already passed through the transition to middle school, and are entering early adolescence. They have capacities and priorities that can serve as either resources or liabilities for motivation. High school may be too late for many interventions, but during eighthgrade it may still be possible to support students' motivational development in time to ease the transition to high school, cultivating motivational resilience.

\section{Markers of Resilience in the Face of the Risks in Eighth Grade}

For students then, moving from sixth- to eighth grade while in a traditional middle school system puts them at risk for successively lower and lower levels of 
motivation and educational success. In order to capture positive adaptation across students' development, it is necessary to mark levels of engagement that have not been negatively affected by the impact of development: What would it look like for an older student to be unaffected by the processes that lead to declines in motivation over time?

Specifically, for a student in eighth grade, at the end of the window of early adolescence, how should resilient motivation be conceptualized? Students' declines in motivation take several steep drops across development; one of the most severe drops occurs, for those in six-to-eighth-grade middle schools, after the transition to middle school and before seventh grade. Thus, resilience for eighth grader in a traditional middle school, unaffected by the negative motivational influences that can come with early adolescence, would be to maintain the motivation of a sixth grader.

Hence, a developmental view of motivational resilience would be one in which students would be identified who are able to maintain the levels of enthusiasm and engagement they held when they completed elementary school and first started middle school. Ideally, longitudinal analyses would allow researchers to identify students whose trajectories of engagement were high and stable across middle school, and compare these to students whose engagement showed average declines and those whose engagement dropped precipitously (a group often labeled "stress-affected"; Luthar, 2006). However, even a cross-sectional design could be useful in this regard, if it allowed cut-off criteria to identify resilient, average, and stress-affected eighth graders to be drawn from comparable populations of fifth- or sixth-grade students. 
By considering the conjunction of demographic risk factors and the vulnerable period posed by adolescent school transitions, one can begin to answer a call posed for a more developmental focus on resilience and the identification of enabling factors (Yates, Egeland \& Sroufe, 2003). Focusing on transitions that happen at certain points in development and the match between students' developmental needs and their environments certainly brings a welcome developmental awareness to the question of educational resilience. The risk that comes when a new school environment does not meet one's changing developmental needs implies that those developmental needs can be considered a potential risk in of themselves.

\section{Developmentally-calibrated Resources for Academic and Motivational Resilience}

Once resilient, average, and stress-affected groups are identified, the remaining task would be to identify which protective and enabling factors should be most important for promoting motivational and academic resilience. Resilience theorists have offered clear and useful guidelines for the selection of protective and enabling factors, stage-environment fit offers a menu of key factors that are salient for adolescents, and SDT offers a well-studied model that supplies candidates for those

factors. By considering the confluence of the developmentally-salient factors proposed by stage-environment fit with those posed by SDT and positive psychology, and housing those factors within a resilience framework, we can arrive at set of factors hypothesized to enable and protect at-risk adolescents' motivational resilience. Specifically, the factors that emerge in this study center around students' opportunities 
for control and decision-making in school, teacher-student relationships, student-student relationships, and academic activities that involve higher-order cognitive processes such as application and finding consequences as a part of classroom work (Eccles \& Midgley, 1989).

\section{Criteria for Selecting Enabling and Protective Factors}

Sonia Luthar (2006), in her review of resilience research, put forth four criteria to guide the selection of protective and enabling factors: Salience, malleability, proximity, and enduringness. Factors, she says, should be "conceptually highly salient" to the particular risk factor. That is, if students' demographic factors and developmental needs combine to put them at risk, mitigating factors should be especially important to the early adolescent experience and should be key issues for low-income, ethnic minority, and immigrant students. Second, a factor should be malleable, something that could be intervened upon. Thus, factors should be parts of the student's system that are open to change. Third, factors should be proximal to the individual, people and ideas with which the students interact directly. Finally, while these factors should be malleable, they should also be enduring; aspects of the students' life that could not only stay relatively stable, but should provide a durable influence on the student's life in the future, and be sustainable.

Martin and Marsh (2009) described three categories for proximal protective factors: 1) Psychological factors such as motivation and a sense of purpose; 2) school and engagement factors such as teacher responsiveness and challenging curriculum; and 
3) family and peer factors. When following Luthar's advice to consider factors most salient to at-risk students' school success in early adolescence, particular psychological, school, and peer factors stand out. Students at this age are learning to navigate their approach to an individual identity, independence, and interdependence.

Psychologically, then, students' sense of autonomy in their schoolwork should be especially important, as well a sense of purpose in school activities. In school, being engaged in authentic, purposeful curricular activities should help channel this desire. Teachers are students' most proximal interaction partners in school. As students' capacities for abstract thought increase, students may begin seeing teachers in a different light and it may become more important that students feel that teachers are responding to their motivational needs. Finally, as students place heightened awareness and importance on their peer relationships, a sense of supportive peers should be an important resource. These three sets of factors seem to be particularly likely candidates for shaping students' motivational resilience during early adolescence.

\section{Enabling Factors within Self-determination Theory}

As discussed, the SSMMD and SDT provide a model for promoting motivation. The framework provided by the SSMMD encapsulates most of the protective and enabling factors commonly used in work on educational resilience (see Morrison et al., 2007, for a list of these factors). Psychological factors, such as self-efficacy, for example, serve a similar function to competence and other self-system processes. When considering an environment of risk, SDT may be particularly useful 
because it assumes a process that rekindles already extant internal resources. Thus, even when students are on a negative trajectory, motivational energy is still posited to be available and SDT models a route for cultivating that motivation. In this sense, the aspects of the SSMMD that predict motivation (contextual factors, nature of academic work, self-system processes) actually actively promote positive adaptation, in addition to softening the impact of negative influences. These factors, then, would best be considered enabling factors rather than exclusively protective factors. However, just as with work on resilience, research on SDT has not yet examined how the promotion of motivational success might be attuned to the specific motivational concerns of eighth grade. Perhaps, as will be explored, there is something unique about the changes that come with early adolescence, bringing a qualitatively different significance of certain aspects of the motivational model and a unique salience of motivational and academic resilience.

\section{Developmental Calibration of Self-Determination Theory}

The self-determination model of engagement has found support across many cultures and contexts, yet there exists almost no information on ways this model might need to be adapted to apply to different ages. Each aspect of the SDT model of engagement, including students' educational contexts, self-perceptions, actions, and outcomes, seems to be important regardless of age, but it is clear that none of these aspects actually remain constant as students age. Just as research has begun to examine different ways that aspects of the model may play out in various cultural contexts (for 
example, expressions of autonomy outside of westernized cultures), it seems vital that research begin to address the different ways that aspects of the SDT model might function during different developmental periods.

In general, each aspect of this model develops and changes along with students as they get older. Students' developmental stages, regardless of what stage that might be, directly shape their internal and external experiences. Developmental progress should inform the make-up and quality of students' environments and contextual partners; the nature of students' interactions with their contexts and how students interpret those interactions; the ways students see themselves and the type of information they find salient to their identities; what it means to be autonomous, competent, and accepted; the complexity of emotions students feel; the scope of actions they take; and whether those behaviors and emotions are categorized by others as engagement or disaffection.

Especially salient in the transition to adolescence are an increased prioritization of peer relationships, and renegotiation of relationships with adult figures, which imply that peers and teachers as contexts may be especially important. The focus during early adolescence on individuality and identity formation calls forth autonomy as an especially important self-system process. Adolescents' increased capacity to conceptualize abstract concepts such as greater meaning and long-term utility may also bring a desire for academic work that is purposeful and authentic (see Error!

\section{Reference source not found.).}


Figure 3.1. Calibration of Self-System Motivational Model of Development: Selected Processes within Schools Salient for Early Adolescents.

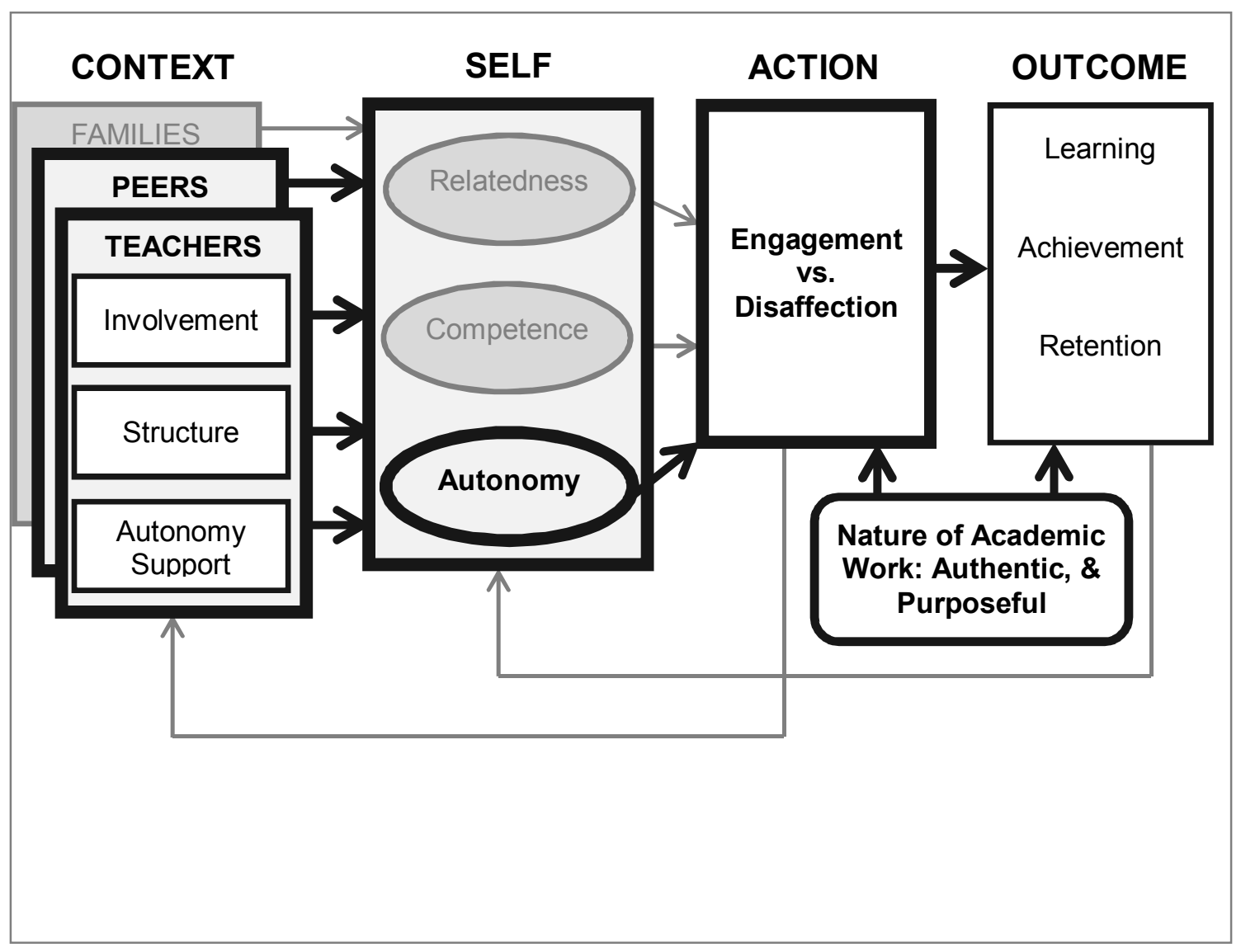

Simply understanding whether these aspects of the model are particularly important for predicting eighth-grade students' resilient motivational and academic development would serve as a needed first step in a larger developmental calibration of the SDT model. Thus, this study examines the aspects of the SSMD that, based on what is known about early adolescents and what has been discovered in work on stage- 
environment fit, should be particularly salient during that age. While all aspects of a students' context, self-perception, action and outcome should be important for all students, students' feelings of being autonomous in school, feelings of support from peers, teachers' attuned support of motivational needs, and academic work that is authentic and purposeful come forward as particular areas of emphasis in this study. The final sections of this chapter review research examining each of these potentially enabling factors.

\section{Enabling Factor 1: Autonomy}

Perhaps the most salient aspect of an early adolescent's development is an increased desire for autonomy. The capacity to reflect on one's own independent thought, emotion, motivation, and behavior informs the intra-and inter-personal changes that eighth graders experience. This autonomous perspective is informed by their increased cognitive and socio-emotional capacities, guides their changing relationships with parents and teachers and increasing prioritization of peers and, and sparks the desire for pursuing one's own interests and utilizing one's capabilities through meaningful and authentic learning activities.

Long-explored as one of the central aspects of adolescence, Erikson (1968) outlined successful development of an autonomous identity as the central task of this developmental stage. The development of independence and interdependence can be seen as mutually important gatekeepers into successful adulthood (Hill, 1980). Several frameworks describe the development and importance of various 
conceptualizations of cognitive, behavioral, and emotional autonomy throughout adolescence (Côté, 2002)

Autonomy within SDT. Much of the prior research on autonomy coalesced with the creation of self-determination theory (Deci \& Ryan, 1985), which holds that the experience of one's self as an autonomous, self-directed person is a primary and universal motivating factor. In this theory, autonomy is the ability to self-organize one's experience and behavior, and to have one's activities be concordant with one's integrated sense of self (Deci \& Ryan, 1985). In a school setting, these central activities are academic tasks, class activities, or homework. Considering that these school activities are often not intrinsically interesting in themselves (Jang et al., 2010), a sense of autonomy in school is generally measured by looking at the reasons that students choose to do these activities.

Some activities are inherently satisfying: Intrinsic motivation reflects a feeling of interest, fun, and enjoyment in activities. For these activities, students need not make themselves do anything; they want to do the activities for their own sakes. However, when considering the activities that come to mind in a standard classroom, it is clear that inherently enjoyable activities are rare. Much of the school experience is seen as onerous, difficult, or boring for students (Anderman \& Maehr, 1994). To some degree, having externally motivated activities, ones done for reasons other than their enjoyment, may be inevitable in schools. Indeed, it seems almost necessary that not all tasks of education can be intrinsically motivating: One cannot learn and grow without 
encountering difficulty. Moreover, the tendency to find challenge enjoyable may come only with experience of successfully regulating one's self through a difficult experience. These types of positive experiences with externalized self-regulation can be understood by examining the range of externalized motivations.

Self-determination theory (Deci \& Ryan, 2000) describes a continuum of externally-motivated reasons for completing tasks, ranging from extrinsic motivation to more autonomous reasons. Extrinsic motivation is a feeling of doing an activity only to avoid getting in trouble, or acting to avoid negative reaction from a teacher. Slightly more autonomous than extrinsic motivation, but still a negative motivational experience, introjected self-regulation reflects internalized feelings of "should:" doing a task to avoid feeling guilt, embarrassment, or other negative feelings. When students are able to take ownership of otherwise onerous activities, however, motivation flourishes. Identified self-regulation is true to its name, describing an incorporation of activities with one's own identity. This more autonomous motivation for one's schoolwork is characterized by a feeling of doing activities because they are "personally important to me," because students find them interesting or relevant (Deci \& Ryan, 2000). In this way, identified regulation fulfills student's inherent needs for autonomy or authentic self-determination, thus sparking their motivation.

In schools, identified self-regulation is easier to come across, and the feeling of ownership that it encapsulates fits well with the questions of identity and personal independence that come with adolescence. To the extent that students can feel that 
school is something that they, personally, care about, students are likely to take responsibility for their own success (Marchand, 2007). Hence, especially useful in early adolescence is the conceptualization of autonomy as identified motivation; the feeling of school activities as being in line with one's own self, encapsulated in a feeling that students themselves are choosing to do activities and that those activities are relevant for students.

Autonomy and engagement. Empirically, several studies have established the link between teacher support of autonomy and academic engagement (See Jang, Reeve, \& Deci, 2010), but far fewer studies look directly at middle school students' perceptions of autonomy as a predictor of engagement (Fredericks, Blumenfeld, \& Paris, 2004). Still, a small body of work (see Table 3.1) has established a strong link between perceived autonomy and engagement for early adolescents (Van Ryzin, Gravely, \& Roseth, 2009) of multiple backgrounds (Connell et al, 1995; Kenny et al, 2010; Tucker, 2000), emerging as an especially strong predictor after controlling for perceptions of one's self as competent and belonging (Skinner, Furrer, Marchand, \& Kindermann 2008; Grolnick et al., 2002). A sense of autonomy is also a strong predictor of engagement after controlling for contextual support from teachers and peers (Van Ryzin et al., 2009), and some evidence suggests that a sense of one's self as autonomous might partially mediate the supportive effects of teachers on students' engagement (Van Ryzin et al., 2009).

In one study, Tucker and colleagues (2002) investigated autonomy in a sample 
of 112 mostly low-income African-American youth (68\% female). In a path analysis with other self-system perceptions and teacher provisions of support as well as grade, perceived autonomy mediated the relationship between teacher autonomy support and engagement. Additionally, researchers found that perceived autonomy uniquely predicted engagement for first through sixth graders after controlling for the strong effects of teacher involvement. Thus, autonomy seems to both capture some aspect of fostering engagement that other self-system processes do not, and to provide a route by which teachers' motivational support aids motivation.

Meeting autonomy needs for older and at-risk students. However, despite the important role autonomy plays as a predictor of engagement, and early adolescents reports that they increasingly desire autonomy in their classrooms (Eccles et al, 1996), there is a striking contrast with the environment that is actually found in schools. As students move from elementary to middle school and from middle school to high school, students perceive schools as being more rigid, less interesting, and providing less autonomy (Eccles et al., 1996). Perhaps in these changes in environment contribute to the consistent declines in students' interest and commitment as they get older (Gottfried 2001). With such a mismatch between students' motivational needs and their environments, one would expect the observed declines in motivation (Silverberg).

These declines are especially strong for ethnic minority and low-income students. Students who are part of an under-represented or stigmatized population, may perceive fewer choices or opportunities, along with an inability to act in accordance 
with one's true self (Steele, 1997; Walton \& Cohen, 2011), and interventions on these types of beliefs sometimes ask students to consider some aspect of their true selves. Perhaps the feeling of autonomy in school, of school being personally important, could serve as just such an aligning resource for students.

Autonomy has been of special interest to those exploring differences between cultures, especially between collectivist and individualistic cultures. While it seems that autonomy is expressed differently across cultures (Chirkov et al. 2009), it does seem to hold as a need that is important to all students. Indeed, one's self-perceptions and motivations seem to be some of the key malleable factors in improving the achievement of at-risk students. Given the particular importance of autonomy to early adolescence and the particular role autonomy plays in school, it seems that any study of early adolescent engagement, especially among disenfranchised young people, will need to include autonomy. 
Table 3.1

Summary of selected research on autonomy predicting engagement and academic outcomes.

\begin{tabular}{|c|c|c|c|c|c|}
\hline \multirow[t]{2}{*}{ Article } & \multicolumn{2}{|r|}{ Sample } & & \multirow[t]{2}{*}{$\mathrm{DV}$} & \multirow[t]{2}{*}{ Findings } \\
\hline & Age & Population & Autonomy & & \\
\hline Connell et al 1994 & $\begin{array}{l}\text { High } \\
\text { school }\end{array}$ & $\begin{array}{l}\text { African American } \\
\text { students }\end{array}$ & $\begin{array}{l}\text { Autonomy from } \\
\text { RAPS-S }\end{array}$ & $\begin{array}{l}\text { Engagement (RAPS- } \\
\text { S, 10-item) }\end{array}$ & Girls (but not boys) Autonomy -> Eng \\
\hline Deci et al 1994 & $6-8$ grade & & $\begin{array}{l}\text { Self-Regulatory Style } \\
\text { Questionnaire }\end{array}$ & $\begin{array}{l}\text { Utilization of various } \\
\text { social partners }\end{array}$ & $\begin{array}{l}\text { Autonomy and Acad. Eng. Vs Disaffect } \\
\text { both related to emulation of significant } \\
\text { adults (teachers \& parents) }\end{array}$ \\
\hline $\begin{array}{l}\text { Grolnick, W. S., } \\
\text { Kurowski, C. O., } \\
\text { Dunlap, K. G., \& } \\
\text { Hevey, C. (2000). }\end{array}$ & $\begin{array}{l}6^{\text {th }} \& 7^{\text {th }} \\
\text { grade }\end{array}$ & 60, urban, $75 \%$ Anglo & Autonomous Self Reg & $\begin{array}{l}\text { Reading \& Math } \\
\text { Performance, } \\
\text { Learning Problems }\end{array}$ & $\begin{array}{l}\text { With perceived comp \& control over } \\
\text { understanding, only aut self reg in } 6^{\text {th }} \\
\text { grade predicted performance } \& \\
\text { decreased problems in } 7^{\text {th }} \text { grade. }\end{array}$ \\
\hline Kenny et al 2010 & $\begin{array}{l}\text { High } \\
\text { school }\end{array}$ & 201, urban & Autonomy support & $\begin{array}{l}\text { Academic } \\
\text { Achievement beliefs }\end{array}$ & $\begin{array}{l}\text { Autonomy support explained unique } \\
\text { variance for ach beliefs }\end{array}$ \\
\hline $\begin{array}{l}\text { Patrick, Skinner, \& } \\
\text { Connell (1993) }\end{array}$ & $\begin{array}{l}3-5 \text { th } \\
\text { grade }\end{array}$ & $\begin{array}{l}246 \text { students, } \\
\text { middle/working class, } \\
\text { mostly White. }\end{array}$ & $\begin{array}{l}\text { Self-Regulatory Style } \\
\text { Questionnaire }\end{array}$ & $\begin{array}{l}\text { Beh \& Emot Eng \& } \\
\text { Disaffection }\end{array}$ & $\begin{array}{l}\text { Unique effects of aut over Control pred } \\
\text { Eng }\end{array}$ \\
\hline $\begin{array}{l}\text { Skinner, Furrer, } \\
\text { Marchand, \& } \\
\text { Kindermann } 2008\end{array}$ & $\begin{array}{l}3-7^{\text {th }} \\
\text { grade }\end{array}$ & $\begin{array}{l}805 ; 2906^{\text {th }} \& 1897^{\text {th }} \\
\text { graders. Middle/working } \\
\text { class, Mostly White }\end{array}$ & $\begin{array}{l}\text { Autonomy Scale (Ryan } \\
\text { \& Connell 1989) } \\
\text { External ->intrinsic. }\end{array}$ & $\begin{array}{l}\text { Beh \& Emot Eng \& } \\
\text { Disaffection } \\
\text { (Skinner/ Wellborn) }\end{array}$ & $\begin{array}{l}\text { Autonomy was strongest predictor } \\
\text { (controlling for control \& relatedness) of } \\
\text { improvements in engagement } \& \\
\text { disaffection over the year. Betas }=(+/-) \\
.42-.56\end{array}$ \\
\hline $\begin{array}{l}\text { Skinner \& Belmont } \\
1993\end{array}$ & $\begin{array}{l}\text { 3-5th } \\
\text { grade }\end{array}$ & 144 children, & $\begin{array}{l}\text { Student \& Teacher } \\
\text { rating of autonomy } \\
\text { support }\end{array}$ & Beh \& Emot Eng & Autonomy support predicts engagment \\
\hline Tucker et al 2002 & $1-12$ & 117, African-American & $\begin{array}{l}\text { RAPS-S: Identified, } \\
\text { Intrinsic, Introjected, } \\
\text { Extrinsic }\end{array}$ & $\begin{array}{l}\text { Engagement (RAPS- } \\
\text { S, 16-item) }\end{array}$ & $\begin{array}{l}\text { Autonomy support and control mediate } \\
\text { teacher supports of those behaviors }-> \\
\text { engagement, beta }=.24 \text {; unique effect } \\
.36 \text { for } 1-6^{\text {th }} \text { graders }\end{array}$ \\
\hline Van Ryzin 2009 & $\begin{array}{l}\text { High } \\
\text { school }\end{array}$ & 283 students, $85 \%$ white, & $\begin{array}{l}\text { Modified Self- } \\
\text { Regulatory Style } \\
\text { Questionnaire }\end{array}$ & $\begin{array}{l}\text { Eng vs. Disaffection } \\
\text { (Skinner \& Furer } \\
\text { 2003) }\end{array}$ & $\begin{array}{l}\text { Autonomy significantly predicts Eng, } \\
\text { over and above peer support and teacher } \\
\text { support, beta =.4 (part of mediational } \\
\text { model - feedback loops, "hope" }\end{array}$ \\
\hline
\end{tabular}




\section{Enabling Factor 2: Teacher Support}

As early adolescents strive for autonomy, they must re-negotiate their relationships with social partners. Steps towards independence from one's parents, if they are be successful, require healthy relationships with adults and other social partners outside the home (Ryan, Stiller, \& Lynch, 1994), such as teachers. A large body of work explores the importance of students' relationships with their teachers for their academic outcomes. A sense of support from one's teachers has been found to predict improved engagement in students (Wentzel, 2009; Roorda et al., 2011; see Table 3.2), including students who are in middle school, who are transitioning into high school, and who are low-income and ethnic minorities. Moreover, many studies have shown that this improved engagement mediates a relationship between teacher support and achievement (e.g., Marchant, Paulson, \& Rothlisberg, 2001; Skinner \& Furrer, 2003). Teacher support not only predicts students engagement, achievement, and learning outcomes, but it feeds back to students own perceptions of themselves (Skinner \& Belmont, 1993).

Why should support from teachers be so important to students? From a very general perspective, interactions with teachers are some of the foundational proximal processes that students encounter in school. Proximal processes, ongoing interactions with others, can be seen as that which drives development, including motivation (Bronfenbrenner \& Morris, 1998). From that perspective, it is difficult to imagine separating teachers from students' motivation and academic development. Moreover, the interactions themselves influence students on multiple levels, including how 
students see themselves, students' motivation and behavior in the classroom, and their actual learning over time. For example, Roeser, Eccles, and Sameroff ( 2000) found that perceptions of teachers support of competence, autonomy, and relationships predicted changes from seventh to eighth grade in students' valuing of academics. Aspects of teacher interactions as varied as the tone of the teacher's communications, his high expectations for the student, his willingness to help, and his support of students' emotional and physical well-being (Wentzel, 2009) have been shown to change students' experiences.

Teacher involvement and student engagement. Self-determination theory and the SSMMD (Deci \& Ryan, 1985, Wellborn \& Connell, 1991) are helpful in characterizing these inter-actions, breaking teacher actions down by how they support students' motivational needs. By letting students feel that somebody cares about them, teachers can meet students' needs for relatedness and belonging (Deci \& Ryan, 2000), influencing students' self-perceptions in a variety of ways, notably promoting a feeling of belonging to school. The importance of teacher warmth, caring, and relationship quality with students has a long history of discussion and empirical support, especially as relates to student engagement (see Wentzel, 2009). Studies from preschool to high school show medium to large effects of the connection between students' relationships with their teachers and their engagement in school (Roorda et al., 2011).

In part because of this sense of relatedness, supportive relationships with teachers help students' engagement in the classroom. Support from teachers promotes positive emotions, helps students feel energized (Martin \& Dowson, 2009; Skinner \& 
Furrer, 2003), and helps them want to follow teacher-given or school-related goals (Wentzel, 2009). For example, in a study of 358 sixth, seventh, and eighth graders, Wentzel and her colleagues (2010) found that teachers' provision of help, emotional support, a safe environment, and positive expectations predicted improvements in how much middle school students enjoyed being in class and cared about what happened in class, with betas ranging from .11 to .30 . In addition to influencing students' emotional engagement in the classroom, teacher support helps students' engaged behavior in class. For example, in a sample of 699 Latino/a middle school students, Brewster \& Bowen (2004) found that when teachers were caring, encouraging, respectful, willing to work with students, and culturally sensitive, students not only liked school more, but had fewer problems with attendance and discipline.

Teacher autonomy support and student engagement. In addition to supporting students' sense of relatedness, teachers' support of students needs for autonomy and competence is important for students' motivation (Ryan \& Deci, 2000; Grolnick et al. 1987; Deci et al. 1996.) Jang, Reeve, \& Deci (2010) synthesized the latest research on what teachers can do to support autonomy: They can nurture inner motivational resources by letting students' own interests, goals, and choices guide instruction; they can provide relevance by communicating rationales and using supportive, non-coercive language; and they can validate students' perspectives by valuing student feelings and expressions, including recognizing negative feelings or criticisms as worthwhile. In this way, autonomy support fosters students' sense that schoolwork is something over which they have some control and would choose to do 
for their own benefit. In other words, their motivation is more autonomous.

As adolescents become more tuned in to their need for autonomy, it would follow that autonomy-supportive teaching would be important for adolescents' motivation. Teachers' provision of autonomy support indeed has been shown to be a consistently strong predictor of engagement in adolescence (See Deci \& Ryan, 2009; Reeve et al, 2010). However, research has now shown that autonomy support is not necessarily more important for adolescents than for younger children (Assor, Kaplan, Roth, 2002). In another study from the same school as the current sample, the aspect of teacher support which was more important to adolescents was actually structure, specifically the perception of justice or fairness (Brule \& Skinner, 2011).

Teacher structure and student engagement. Nor is autonomy the only important way for teachers to facilitate adolescents' motivation. Recent research has recategorized structure, the support of students' needs for competence, as complementary to autonomy support and, therefore, to overall motivation (Jang, Reeve, \& Deci, 2010). This research nudges the field away from separate models of support that, for example, placed autonomy support and structure at opposite ends of a controlling behavior spectrum, and towards a model in which all kinds of teacher support foster all kinds of self-system perceptions. For example, Tucker and colleagues (2011) found in a path analysis that teacher provision of structure promoted engagement through a sense of relatedness, not competence. Thus, an integrated conceptualization of teacher support, incorporating autonomy support, structure, and relatedness, seems to be the most relevant in supporting motivation. 
As students get older, this kind of integrated support allows students to feel that their motivational needs are being met, even if those needs are changing. For an adolescent to perceive a teacher as being autonomy supportive, he probably requires a different set of actions than a younger student might, for example. Age-inappropriate behavior - being condescending, or not trusting students to complete tasks, for example - translates into feelings of coercion or rejection for students. To some extent, studentreports of motivational support from teachers can be interpreted as a marker of how attuned teachers are to students' developmental needs.

Teacher support for older and at-risk students. Despite the importance of teacher support in adolescence, students tend to report less support from teachers as they get older. Eccles and her colleagues $(1993,1996)$ have explored some of the factors underlying this perception: Not only teachers, but the entire academic environment is seen as less supportive as students get older. Perhaps because teacher support becomes more of a scarce resource, some research suggests that teacher support may be a stronger predictor of engagement as students get older (Furrer \& Skinner, 2003; Marchant et al., 2001; Skinner et al., 1998). Similarly, a variety of research has shown that teacher support is both observed in lower levels and may more important for student success for students who are low-income (Dornbusch et al, 2001) and members of ethnic minorities (Crosnoe et al, 2004). For at risk-adolescents, then, teacher support can be considered a vital resource. 
Table 3.2

Summary of selected research linking teacher support and engagement.

\begin{tabular}{|c|c|c|c|c|c|}
\hline \multirow[t]{2}{*}{ Article } & \multicolumn{2}{|r|}{ Sample } & \multirow[t]{2}{*}{ Measure of Teacher Support } & \multirow{2}{*}{$\begin{array}{c}\text { Measure of } \\
\text { Engagement }\end{array}$} & \multirow[t]{2}{*}{ Findings } \\
\hline & Age & n, Population & & & \\
\hline \multicolumn{6}{|c|}{ Involvement, Structure, and Autonomy Support } \\
\hline $\begin{array}{l}\text { Furrer \& } \\
\text { Skinner } 2003\end{array}$ & $\begin{array}{l}3-6^{\text {th }} \\
\text { graders }\end{array}$ & $\begin{array}{l}641,95 \% \text { White, } \\
\text { middle/working } \\
\text { class, rural New } \\
\text { York }\end{array}$ & $\begin{array}{l}4 \text { items: When I'm with my } \\
\text { teacher, I feel accepted/important }\end{array}$ & $\begin{array}{l}\text { Behavioral \& emotional } \\
\text { engagement vs. } \\
\text { Disaffection, student and } \\
\text { teacher report }\end{array}$ & $\begin{array}{l}\text { Relatedness predicts teacher } \\
\text { report (TR) and student report } \\
\text { (SR) of engagement } \\
\text { controlling for perceived } \\
\text { control, and controlling for } \\
\text { parents and peers. } \\
\text { Engagement (both TR \& SR) } \\
\text { mediates relationship between } \\
\text { teacher relatedness \& } \\
\text { achievement. Relatedness } \\
\text { predicts spring engagement, } \\
\text { controlling for fall, and } \\
\text { predicts changes fall to spring; } \\
\text { is a stronger predictor of } \\
\text { engagement for boys (beta .24) } \\
\text { than girls (.24); and of } \\
\text { behavioral engagement (TR) } \\
\text { for older children (drop in } \\
\text { mean levels after } 6 \text { th grade). }\end{array}$ \\
\hline
\end{tabular}




\begin{tabular}{|c|c|c|c|c|c|}
\hline $\begin{array}{l}\text { Klem \& } \\
\text { Connell }\end{array}$ & $\begin{array}{l}\text { Middle } \\
\text { school }\end{array}$ & $\begin{array}{l}2430,45 \% \text { African } \\
\text { American, } 39 \% \\
\text { Euro American, } \\
16 \% \text { Hispanic, } \\
58 \% \text { low-income }\end{array}$ & $\begin{array}{l}\text { Student experiences of Teacher } \\
\text { support, } 14 \text { items: involvement, } \\
\text { structure, and autonomy support }\end{array}$ & $\begin{array}{l}\text { Student reports of } \\
\text { Engagement from RAPS-S: } \\
\text { Ongoing Engagement \& } \\
\text { Reaction to Challenge, } 11 \\
\text { items; Teacher report of } \\
\text { (beh) Engagement, } 3 \text { items }\end{array}$ & $\begin{array}{l}\text { Created optimal \& risk } \\
\text { thresholds; Those with low } \\
\text { teacher support } 68 \text { percent } \\
\text { more likely to be disengaged } \\
\text { from school, } 71 \text { percent less } \\
\text { likely to be engaged; Those } \\
\text { with high levels } 47 \% \text { likely to } \\
\text { be engaged (TR) and } 47 \% \text { less } \\
\text { likely to be disengaged. } \\
\text { Presence of teacher support } \\
\text { more important in predicting } \\
\text { Engagement for middle- } \\
\text { schoolers than elementary }\end{array}$ \\
\hline $\begin{array}{l}\text { Marchant et } \\
\text { al. } 2001\end{array}$ & $\begin{array}{l}5^{\text {th }} \& 6^{\text {th }} \\
\text { graders }\end{array}$ & $\begin{array}{l}230,92 \% \text { white, } \\
\text { middle/working } \\
\text { class }\end{array}$ & $\begin{array}{l}\text { Structure \& Relatedness with } \\
\text { Teacher control (perceptions of } \\
\text { rules \& discipline: strict/freedom), } \\
\text { Teacher Responsiveness (interest } \\
\text { in \& support of students: my } \\
\text { teachers believes I have a right to } \\
\text { my own opinion/makes me feel } \\
\text { good about what I achieve) from } \\
\text { Rutter } 1983 .\end{array}$ & $\begin{array}{l}\text { Motivations scale: } 5 \text { items } \\
\text { on importance of ability, } \\
\text { effort, \& grades. }\end{array}$ & $\begin{array}{l}\text { Motivations \& school } \\
\text { competence mediated impact } \\
\text { of teacher support on } \\
\text { achievement (teachers } \\
\text { responsiveness predicting } \\
\text { motivation, beta }=.16 \text { ) }\end{array}$ \\
\hline $\begin{array}{l}\text { Reeve, Jang, } \\
\text { Carrell, } 2004\end{array}$ & $\begin{array}{l}\text { High } \\
\text { School }\end{array}$ & $\begin{array}{l}\text { Unknown (focus } \\
\text { on teachers); } 20 \\
\text { teachers and } \\
\text { classrooms in the } \\
\text { Midwest. }\end{array}$ & $\begin{array}{l}\text { Classroom observations, rating } \\
\text { aspects of autonomy support ( } 4 \\
\text { items; motivational resources, } \\
\text { controlling vs informational } \\
\text { language, relevance, reaction to } \\
\text { negative affect), Relatedness (4 } \\
\text { items; warmth, personal resources, } \\
\text { physical proximity, knows } \\
\text { students) Structure (5 items; clear } \\
\text { directions, leadership/goals, } \\
\text { challenging, scaffolding, } \\
\text { feedback) }\end{array}$ & $\begin{array}{l}\text { Classroom observations } \\
\text { rating aspects of classroom- } \\
\text { level collective engagement } \\
\text { (5 items; attention, } \\
\text { effort/activity, verbal } \\
\text { participation; giving } \\
\text { up/persisting and emotional } \\
\text { tone during challenge or } \\
\text { confusion) }\end{array}$ & $\begin{array}{l}\text { Experiment, observations. } \\
\text { Teachers trained in autonomy } \\
\text { support provided more } \\
\text { autonomy support than } \\
\text { untrained teachers; Autonomy } \\
\text { support predicted engagement } \\
\text { over and above involvement } \\
\text { and structure; autonomy } \\
\text { support a better predictor of } \\
\text { student engagement in later } \\
\text { sessions than students' prior } \\
\text { engagement (betas }=.54-.61 \text { ) }\end{array}$ \\
\hline
\end{tabular}




\begin{tabular}{|c|c|c|c|c|c|}
\hline $\begin{array}{l}\text { Ryan, Stiller, } \\
\text { \& Lynch } 1994\end{array}$ & $\begin{array}{l}7^{\text {th }} \& 8^{\text {th }} \\
\text { graders }\end{array}$ & $\begin{array}{l}606, \text { Rochester } \\
\text { suburb. }\end{array}$ & $\begin{array}{l}\text { Felt-security } \\
\text { (affective/attachment) \& } \\
\text { emotional utilization (rely on } \\
\text { during emotional situations) from } \\
\text { IAA, Greenberg 1982. School } \\
\text { utilization (rely on for help w/ } \\
\text { school), Emulation (identify with/ } \\
\text { want to be like). }\end{array}$ & $\begin{array}{l}\text { Academic engagement vs. } \\
\text { disaffection (Wellborn \& } \\
\text { Connell, 1987). }\end{array}$ & $\begin{array}{l}\text { All four aspects significantly } \\
\text { predicted engagement } \\
\text { (Security: Beta }=.32 \text {; Rest: } \\
\text { Betas } .14-.19, p<.001 \text { ) }\end{array}$ \\
\hline $\begin{array}{l}\text { Skinner, } \\
\text { Furrer, } \\
\text { Marchand, \& } \\
\text { Kindermann } \\
2008\end{array}$ & $\begin{array}{l}3-7^{\text {th }} \\
\text { grade }\end{array}$ & $\begin{array}{l}805 ; 2906^{\text {th }} \& 189 \\
7^{\text {th }} \text { graders. } \\
\text { Middle/working } \\
\text { class, Mostly } \\
\text { White }\end{array}$ & $\begin{array}{l}\text { Student report: Involvement (9 } \\
\text { items), Structure ( } 21 \text { items), \& } \\
\text { Aut Sup (18 items). Teacher } \\
\text { report: Involvement (14 items), } \\
\text { Structure (5 items), Autonomy } \\
\text { Support ( } 12 \text { items), Skinner \& } \\
\text { Belmont } 1993 .\end{array}$ & $\begin{array}{l}\text { Beh \& Emot Eng \& } \\
\text { Disaffection (Skinner/ } \\
\text { Wellborn) }\end{array}$ & $\begin{array}{l}\text { TR predicted improvements in } \\
\text { behavioral engagement; SR } \\
\text { predicted improvements in } \\
\text { behavioral \& emotional } \\
\text { engagement \& disaffection, } \\
\text { controlling for fall levels, } \\
\text { betas }=+/-.10-.24 . \text { SR fully } \\
\text { mediated relationship between } \\
\text { TR \& Engagement. }\end{array}$ \\
\hline $\begin{array}{l}\text { Tucker, et al. } \\
2002\end{array}$ & $\begin{array}{l}1^{\text {st }}-12^{\text {th }} \\
\text { grade }\end{array}$ & $\begin{array}{l}117 \text { low-income } \\
\text { African American } \\
\text { students. }\end{array}$ & $\begin{array}{l}\text { RAPS-S; autonomy support (7 } \\
\text { items), }\end{array}$ & $\begin{array}{l}\text { RAPS-S; } 16 \text { items } \\
\text { assessing emotional } \\
\text { engagement ( } 6 \text { items), } \\
\text { centrality of school ( } 1 \\
\text { item), effort ( } 4 \text { items), } \\
\text { attention ( } 2 \text { items), beyond } \\
\text { the call ( } 3 \text { items) }\end{array}$ & $\begin{array}{l}\text { Combined teacher support } \\
\text { predicted engagement (beta }= \\
.50 \text { ) controlling for grade, but } \\
\text { only involvement predicted } \\
\text { when controlling for other } \\
\text { teacher supports, and when } \\
\text { controlling for SSPs. }\end{array}$ \\
\hline $\begin{array}{l}\text { Van Ryzin } \\
2011\end{array}$ & $\begin{array}{l}7^{\text {th }}-11^{\text {th }} \\
\text { Grade }\end{array}$ & $\begin{array}{l}423 \text {, at } 5 \text { schools in } \\
\text { Midwest; } 78 \% \\
\text { White, } 30 \% \\
\text { reduced lunch }\end{array}$ & $\begin{array}{l}\text { "Autonomy," } 4 \text { items from } \\
\text { Learning Climate Q. (Williams \& } \\
\text { Deci 1996), "my teachers . . . } \\
\text { provide me choices" “...listen to } \\
\text { how I would like to do things"; } \\
\text { Teacher support from Classroom } \\
\text { Life Scale (Johnson et al. 1985), } 4 \\
\text { classroom academic items } \\
\text { (“... want me to do my best”), } 4 \\
\text { personal support items (“ ...care } \\
\text { about me”) }\end{array}$ & $\begin{array}{l}\text { Engagement vs. } \\
\text { Disaffection with Learning } \\
\text { Scale (Patrick/Skinner); } \\
\text { beh \& emot combined into } \\
\text { one score, alpha }=.86\end{array}$ & $\begin{array}{l}\text { Autonomy, but not Teacher } \\
\text { Support, predicted } \\
\text { Engagement, controlling for } \\
\text { previous year's engagement, } \\
\text { Peer support, mastery goal } \\
\text { orientation, \& performance } \\
\text { goal orientation. (Eng. In } \\
\text { previous year was a sig } \\
\text { predictor of teacher support) } \\
\text { Au }\end{array}$ \\
\hline
\end{tabular}




\begin{tabular}{|c|c|c|c|c|c|}
\hline $\begin{array}{l}\text { Wentzel et al. } \\
2010\end{array}$ & $\begin{array}{l}6^{\text {th }}, 7^{\text {th }} \\
8^{\text {th }} \\
\text { graders }\end{array}$ & $\begin{array}{l}358,75 \% \text { White, } \\
22 \% \text { African } \\
\text { American, } 7 \% \\
\text { reduced lunch }\end{array}$ & $\begin{array}{l}3 \text { items each: Emotional support, } \\
\text { Safety (criticism), Help, } \\
\text { Expectations for positive social } \\
\text { behavior (wants me to share } \\
\text { ideas). Expectations for } \\
\text { Academic Engagement (and for } \\
\text { learning }\end{array}$ & $\begin{array}{l}\text { Interest (enjoy being in } \\
\text { class/don't care what } \\
\text { happens in class) }\end{array}$ & $\begin{array}{l}\text { Interest predicted by } \\
\text { expectations (beta }=.18) \text {, } \\
\text { safety }(.11) \text {, help }(.30), \text { and } \\
\text { emotional support }(.20) \\
\text { Various grade by gender } \\
\text { interactions, not a unified } \\
\text { pattern. (Their } 7^{\text {th }} \text { graders were } \\
\text { weird also). }\end{array}$ \\
\hline $\begin{array}{l}\text { Zimmer- } \\
\text { Gembeck et al } \\
2006\end{array}$ & $10^{\text {th }}$ & $\begin{array}{l}324, \text { middle-class } \\
\text { ish, } 80 \text { percent } \\
\text { White }\end{array}$ & $\begin{array}{l}\text { Student's Assessment of Teacher } \\
\text { Context (Belmont, Skinner, } \\
\text { Wellborn, Connell 1992): Aut } \\
\text { sup, inv, structure }\end{array}$ & $\begin{array}{l}\text { Behavioral \& Emotional } \\
\text { engagement \& Disaffection } \\
\text { (Skinner 1998) }\end{array}$ & $\begin{array}{l}\text { Teacher Support predicts } \\
\text { Engagement, beta }=.69, \\
\text { controlling for peer support. }\end{array}$ \\
\hline \multicolumn{6}{|c|}{ Focus on at- Risk Students } \\
\hline $\begin{array}{l}\text { Brewster \& } \\
\text { Bowen, } 2004\end{array}$ & $\begin{array}{l}1896-8 \\
\text { graders, } \\
4449- \\
12 \\
\text { graders }\end{array}$ & $\begin{array}{l}699 \text { Latino/a } \\
\text { students from } \\
\text { schools across US, } \\
65 \% \text { low-income, }\end{array}$ & $\begin{array}{l}7 \text { items assessing caring, } \\
\text { encouraging, respect, willing to } \\
\text { work with students: "listen to } \\
\text { what I have to say/care whether I } \\
\text { come to school/understand racial } \\
\text { \& cultural differences" }\end{array}$ & $\begin{array}{l}\text { "Problem behavior in } \\
\text { school" (Attendance \& } \\
\text { discipline; DeVellis, 1991) } \\
\text { and "school } \\
\text { meaningfulness" ("I find } \\
\text { school fun and exciting/I } \\
\text { look forward to coming to } \\
\text { school;" Bowen, 1998) }\end{array}$ & $\begin{array}{l}\text { Teacher support significantly } \\
\text { influenced problem behavior } \\
\& \text { school meaningfulness, } \\
\text { controlling for parental } \\
\text { support \& demographic } \\
\text { factors. School level (MS vs } \\
\text { HS) had no sig. main effect in } \\
\text { step } 1 \text { (demographics) }\end{array}$ \\
\hline $\begin{array}{l}\text { Ryan, A. M. } \\
\text { \& Patrick, H, } \\
2001\end{array}$ & $\begin{array}{l}7^{\text {th }} \text { into } \\
8^{\text {th }} \text { grade }\end{array}$ & $\begin{array}{l}233,55 \% \text { African- } \\
\text { American, } 45 \% \\
\text { White, } 4 \% \text { low } \\
\text { income }\end{array}$ & $\begin{array}{l}\text { Teacher support \& promoting } \\
\text { teacher-student relationships, from } \\
\text { Teacher Support subscale of } \\
\text { Classroom Environment scale } \\
\text { (Moos \& Trickett, 1974) }\end{array}$ & $\begin{array}{l}\text { Student motivation } \\
\text { (academic efficacy), } \\
\text { student engagement (self- } \\
\text { regulated learning), } \\
\text { disruptive behavior }\end{array}$ & $\begin{array}{l}\text { Teacher support related } \\
\text { positively to academic efficacy } \\
\& \text { self-regulated learning, } \\
\text { negatively to disruptive } \\
\text { behavior (beta }=-.21, p<.001 \text { ). }\end{array}$ \\
\hline \multicolumn{6}{|c|}{ Focus on Autonomy and Structure } \\
\hline $\begin{array}{l}\text { Assor, } \\
\text { Kaplan, \& } \\
\text { Roth, } 2002\end{array}$ & $\begin{array}{l}6-8^{\text {th }} \\
\text { grade }\end{array}$ & $\begin{array}{l}\text { 364 Israeli-Jewish } \\
\text { (Study in Israel); } \\
\text { middle - lower } \\
\text { middle-class }\end{array}$ & $\begin{array}{l}34 \text { Autonomy items from RAPS } \\
\text { (Connell \& Wellborn 1990). } \\
\text { Differentiated three types of } \\
\text { autonomy support (relevance, } \\
\text { allowing criticism, choice) and } \\
\text { autonomy suppression } \\
\text { (suppressing criticism, intruding, } \\
\text { unmeaningful acts) }\end{array}$ & $\begin{array}{l}6 \text { items, behavioral \& } \\
\text { Cognitive engagement in } \\
\text { schoolwork -“" pay } \\
\text { attention"/"try to } \\
\text { understand"/"do more than } \\
\text { required in classes taught } \\
\text { by X". }\end{array}$ & $\begin{array}{l}\text { Suppression of criticism (beta } \\
=-.15 \text { ) and fostering relevance } \\
\text { (.24) predicted engagement, } \\
\text { controlling for other types } \\
\text { (unmeaningful activities } \\
\text { related to feelings but not out } \\
\text { engagement). Students able to } \\
\text { differentiate different types of } \\
\text { autonomy support. }\end{array}$ \\
\hline
\end{tabular}




\begin{tabular}{|c|c|c|c|c|c|}
\hline $\begin{array}{l}\text { Jang, Reeve, } \\
\text { Ryan, \& Kim, } \\
2009\end{array}$ & $\begin{array}{l}10^{\text {th }} \\
\text { grade }\end{array}$ & $\begin{array}{l}256 \text { (study 1), } 272 \\
\text { (Study 2, different } \\
\text { sample) middle- } \\
\text { class, urban, South } \\
\text { Korea }\end{array}$ & $\begin{array}{l}\text { Autonomy Support: } 8 \text { items from } \\
\text { Learning Climate Questionnaire } \\
\text { (Williams et al, 1996), assessing } \\
\text { choices, teacher listening to } \\
\text { suggestions, etc. External } \\
\text { Control: } 4 \text { items from Teacher } \\
\text { Control Questionnaire (Jeon, } \\
\text { 2004): Teachers' } \\
\text { controlling/pressuring actions. }\end{array}$ & $\begin{array}{l}14 \text { items; class involvement } \\
\& \text { task persistence, based } \\
\text { on Wellborn } 1991\end{array}$ & $\begin{array}{l}\text { Autonomy support vs. external } \\
\text { control predicted Autonomy } \\
(.47) \text { and Competence }(.40) \text {, } \\
\text { which in turn predicted } \\
\text { Engagement }(.39 \& .23) \text {. }\end{array}$ \\
\hline $\begin{array}{l}\text { Jang, Reeve, } \\
\& \text { Deci } 2010\end{array}$ & $\begin{array}{l}9^{\text {th }}-11^{\text {th }} \\
\text { grade }\end{array}$ & $\begin{array}{l}2,523 \text { students at } 9 \\
\text { schools and } 133 \\
\text { teachers. Students } \\
47 \% \text { Caucasian, } \\
45 \% \text { African } \\
\text { Amerian, } 20 \% \\
\text { reduced lunch }\end{array}$ & $\begin{array}{l}\text { Observer ratings (Reeve, Jang et } \\
\text { al 2004) of autonomy support ( } \\
\text { nurtures motivational resources, } \\
\text { informational language, } \\
\text { acknowledges negative affect) and } \\
\text { structure (clear directions, strong } \\
\text { guidance, constructive feedback; } \\
\text { Skinner \& Belmont, 1993) }\end{array}$ & $\begin{array}{l}\text { Observer ratings of } \\
\text { classroom-level collective } \\
\text { engagement: attention, } \\
\text { effort, verbal participation, } \\
\text { persistence, positive } \\
\text { emotion (Skinner \& } \\
\text { Belmont 1993), and Voice } \\
\text { (DeCharms); as well as } \\
\text { student-report behavioral, } \\
\text { cognitive, and emotional } \\
\text { engagement (Fredericks et } \\
\text { al. 2004). }\end{array}$ & $\begin{array}{l}\text { Structure and Autonomy } \\
\text { support positively related but } \\
\text { separate factors. Ratings of } \\
\text { structure and autonomy } \\
\text { support predict collective } \\
\text { engagement }(.36 \& .38) \text { but } \\
\text { only autonomy support } \\
\text { predicted self-report } \\
\text { engagement (.19) when } \\
\text { controlling for autonomy } \\
\text { support. }\end{array}$ \\
\hline
\end{tabular}




\section{Enabling Factor 3: Peer Support}

As young people re-organize their social structures during adolescence, beginning to form their own networks, peers take a more central role in students' lives (Ryan \& Patrick, 2001). With this increasing importance, peers take on a greater influence in students' lives as well. Adolescents tend to rely on their peers more, and hold their opinions in higher esteem (Ryan, Stiller, \& Lynch, 1994). Meanwhile, peers are a numerical majority among influential partners in students' school environments (Hymel, Comfort, Schonert-Reichl, \& McDougall, 1996), and play a key role in students' developing identities (Roeser et al., 2008). Just as with teachers, peers are an important context within the self-system, and support from peers should foster students' self-perceptions, bolstering their engagement (Furrer, Skinner, \& Pitzer, 2014). However, despite the important role peers play in adolescence, the research on peers support of students' academic experiences is much less-developed than that on parents and peers (Ryan, 2000).

Peer relationships and academic outcomes. Although not as cohesive as research on teachers, plentiful research links peers to students' motivational and achievement outcomes (see Ladd, Herald-Brown, \& Kochel, 2009; Ryan, 2001). Through varied lines of research, these links between peers and academic outcomes differ based on outcome (e.g., social goals, academic value, achievement; Wentzel \& Caldwell, 1997), type of peer relationship (e.g. recipriocal friendships, co-nominated peer groups; Wentzel, 2008), type of interactions (e.g. acceptance, rejection, identification; Ladd et al., 2009), as well as by what characteristics of the peer is 
measured. Research looks at, for example, peers' popularity, positive and negative behaviors, and their respective levels of engagement; much of the research on peers and engagement examines the extent to which peers influence one another's motivation via contact in social networks (e.g. Kindermann, 2007). However, when considering peers as a motivational resource in regard to the developmental needs of students, of most interest are peers as a context of social support and interaction (e.g. Wentzel, 2010). The amount of research that directly connects adolescent students' ratings of supportive peer relationship and academic engagement (e.g., Marks, 2011), is smaller than one might expect (Fredricks et al., 2004; Ladd et al., 2009). One possibility for the small number of these studies may be that perceptions of peer support do not come online as a strong predictor of motivation until students are in early adolescence. In a prior analysis using the same dataset as the current study, there was a significant interaction between grade and peer support when predicting engagement, such that peer support was a stronger predictor eighth graders than younger students (Brule \& Skinner, 2011), and, when looking at simple slopes, there was no significant relationship for sixth graders.

Peer support as a motivational resource or liability. Although positive relationships with peers have been shown to positively influence students' motivation and academic success, much of the research on peers traditionally explores the negative ways that peers can influence students' school lives. For example, research has shown that students who lack a sense of peer support and who feel rejected also lack engagement (Fredricks et al., 2004). This makes sense, when considering that peer 
support should be a motivational resource. If peers are a necessary part of students' motivational experience, missing that ingredient should inhibit motivation. Indeed, it is not uncommon for something that is a protective factor at a high level to be a risk factor at a low level (Luthar, 2006).

When considering that peers bolster motivation, supportive peer relationships should give students reasons to come to, or avoid coming to, school. For example, in a sample of 358 sixth, seventh, and eighth graders, Wentzel (2011) found that perceptions of help, emotional support, and safety from peers predicted academic interest and commitment. Whether a student is looking forward to seeing friends, dreading a negative interaction, simply feels that the people at the school are likeable, that perception of supportive peer relationships should influence the extent to which that student feels a connection and commitment to school, and thus his engagement and achievement.

Peer support with older and at-risk students. If demographically young people do not see academically successful peers or role models, they may decide that academic success is not an option for people like them (Oyserman \& Bybee, 2006). Research in the areas of possible selves and belonging uncertainty explore the important role peers play in ethnic minority and low-income students' academic development. In this way, peer support may be more important for at-risk students whose perceptions of acceptance or fitting in in school may be at higher risk. To effectively promote engagement for all students, one must consider the presence of and consider how to foster supportive interactions with students' peers. 


\section{Enabling Factor 4: Authentic and Purposeful Learning: Engagement in Garden- Based Education}

As autonomy becomes more salient (Anderman \& Maehr, 1994) during adolescence, the nature of academic work (Wigfield et al. 2006) should correspondingly become more important. If students are increasingly driven by their own individual identities, it would make sense that their engagement with schoolwork would depend on the work's actual relation to students' identities. Indeed, research shows that students' interest in and valuing of work are increasingly important as adolescents get older (Wigfield \& Eccles, 1992). However, just during the time when students desire more personal importance in their schoolwork, academics become less interesting and students report being more bored (Wigfield et al., 2006). Academic work in middle school tends to become more abstract and evaluation-focused (Eccles et al., 1996), and students experience their schoolwork as lacking both personal and cultural meaning (Wigfield et al 2006).

Efforts to increase contextualized learning, rooted in hands-on, project-based attempts to infuse relevance in abstracted learning environments, but hands-on activities may not be enough to bolster students' motivation if the activities fail to carry a sense of personal meaning for students. If a student is immersed in a real-world task that she finds unimportant, the added relevance would be unlikely to help her feel more committed or curious about the task. In that way, a sense that one's schoolwork is purposeful may be a key to successful contextual learning. Nearly a century before this study, theorists raised the same call: "The unifying idea [of project-based education] 
was that students learn best when 'wholeheartedness of purpose is present"' (Kilpatrick, 1918)

Moreover, developmental research has begun to include a sense of purpose as one of the key developmental tasks of adolescence: Young people must establish not only an identity, but a direction to that identity. Perhaps because of its guiding nature, purpose seems to be an important factor in adolescents' resilience (Van Dyke \& Elias, 1997). In understanding not only how to support engagement but how to support eighth graders' maintenance of engagement despite the odds, it is important to understand the factors that help buoy middle school students, and purpose may be an especially important factor for early adolescents.

Purpose as a motivational resource. Meaning or purpose as a motivational resource was introduced by Viktor Frank1 (1959) in his classic work “Man's search for Meaning”. Working towards a purpose, he argued, was the essential source of resilience in prisoners of the World War II Nazi Concentration camps: Those who maintained a will to survive were those who had a contribution they needed to make in the world. The Purpose in Life (Butler \& Carr, 1968) scale was one of the first measures of sense of purpose, and has since been used sporadically in educational assessments. Frankl's death in 1997 sparked a small resurgence of research on purpose, including an investigation of the importance of meaning and purpose in adolescence. This research has begun to cement purpose as a key developmental task of adolescence (Damon, 2008).

Current work on purpose generally uses a conceptualization of true purpose as 
containing four parts: Something that is purposeful must be important to the self, impact others, be something on which a person both can take current action and can see as a part of a long term arc (Damon, Menon \& Bronk, 2003). That is, a purpose that is either entirely selfless or entirely selfish will not provide the same benefits as one that is both personally fulfilling and serves others. At the same time, a purpose that is a onetime affair, disconnected from any sense of growth or future-orientation, may be just as dissatisfying as a purpose that lives purely in the future, without any way to engage with it directly in the present.

Considering the implications underlying these requirements, it makes sense that purpose might become more important in adolescence. To balance a purpose that is both self-fulfilling and other-oriented, one must have both an idea of one's own self and the capacity to balance independence with interdependence. The ability to balance long-term goals and short-term engagement requires sophisticated abstract thought. A small number of researchers (Fry, 1998) have discussed how the cognitive and socioemotional gains experienced in adolescence are essential to not only the capacity but also the desire for meaning and purpose. Indeed, some argue that purpose is the core ingredient of Erikson's conceptualization of identity development (Hill \& Burrow, 2012)

Research on students has established that early adolescents have purposes and think about them in a way consistent with other work on purpose (Hill, Burrow, O'Dell, \& Thornton, 2010). Purpose, with its galvanizing and orienting nature, may be particularly important for at-risk students (Kiang \& Fuligni, 2010) whose environments 
may exert a pull away from healthy behaviors. Indeed, from its conception, work on purpose has examined the role purpose can serve in supporting resilience in the most dire situations of risk, offering a route to competent adaptation by literally make meaning out of treacherous circumstances (Van Dyke \& Elias 1997; Frankl 1959).

It follows, then, that purpose would be an important resource in promoting motivational resilience. Indeed, purpose has emerged as a key route for promoting motivation and resilience for at-risk adolescents, as demonstrated both via qualitative (Dawes \& Larson, 2011) and meta-analytic (Morrison et al., 2011) investigations. One way to support this resilience, then, should be by providing students with the types of activities that should help them feel purposeful (Yeager \& Bundick, 2009).

Garden-based education. Garden-based education is one especially promising context for the type of purposeful academic work that could serve as a motivational resource for students. Schools everywhere are implementing school gardens, with thousands in the United States (Blair, 2009) and across the world. Gardens are integrative, hands-on, real-world learning settings (Ozer, 2007). Moreover, gardening is naturally purposeful, and purpose-promoting (Fusco, 2001).

Gardens have real-world impacts on several levels. At perhaps the most basic and universally appealing, gardens produce food that students get to eat. When situated within schools, however, gardens take on diverse sophisticated meanings. In areas where food security is a pressing concern, school gardens can directly assist the families of their students, as well as give young people a chance to be responsible for food donated to food banks (Blair, 2009). Gardens have given young people a chance to 
revitalize neighborhoods, improving ownership and physical safety in their communities (Blair, 2009). In school gardens, some of the most abstract concepts from biology and chemistry are translated into environmental action, emotionally hot processes that can give young people a chance to combat environmental destruction. These larger impacts of gardening fulfill in an exemplary fashion the required beneficiaries of a purposeful activity - both self and other - posed by Damon and his colleagues (2003). Gardening can help individuals, families, communities, and the world.

When considering Damon's other two requirements for a purposeful activity, that it offer a long-term arc and an opportunity for immediate action, the activities in gardening again offer special promise. Few things, perhaps, so tangibly demonstrate the potential payoff of long-term projects as a student planting a seed, helping it grow, and seeing his work come to literal fruition (Fusco, 2001). Moreover, the immediate physical and psychological experience of gardening offers fulfillment that students often miss in traditional schooling, from simply being outside (Ryan et al. 2010) to using one's body and creativity.

These myriad potential benefits seem to really help students' academic experiences. A relatively small body of research has shown that, indeed, gardens can help students become energized (Blair, 2009) and interested (Desmond et al., 2002) in school. In particular, gardens can stoke students' enthusiasm for learning, bolstering their interest in science (Dirks \& Orvis, 2005), and predicting improvements in both their academic engagement and learning (Skinner \& Chi, 2012). 
Garden-based education may offer special benefits for at-risk middle school students. It has been clear that students from diverse ethnicities and incomes seem to benefit from garden-based education (Fusco, 2001; Bowker \& Tearle, 2007; Blair, 2009; Ratcliffe, et al., 2010). Moreover, across dozens of schools, gardening seemed to consistently explain a reduction the achievement gap (Leiberman \& Hoody, 1998).

Garden-based education shows immense promise as an avenue for authentic, purposeful learning, and is already a widespread part of schools. Perhaps the most striking thing to emerge from research, however, is the lack of cohesive findings. While many educators that gardening is a positive addition to schools, very little is known about the active ingredients of garden-based education. Initial studies addressing this question have shown that students' engagement in gardens helps explain the academic benefits brought by gardening (Skinner, Chi, \& the LEAG, 2012). This makes sense: Simple participation in gardens would not help students for whom gardening isn't seen as purposeful, important, or at least fun: Their engagement is vital, and feelings of engagement capture much of the essence of gardening's proposed benefits. In order to assess whether garden-based education can be a motivational resource, one should assess students' engagement in a gardening program that is organized as a purposeful learning opportunity.

Gardening as sustainable education and action. The current study measured students' participation in a garden program designed to espouse principles of sustainable education. In a collaboration between a university graduate education program and a middle school, graduate students in Leadership for Sustainability serve as Garden 
Educators, leading weekly gardening classes with middle school students as a part of students' regular science classes. From sustainability pedagogies, the Garden Educators base education on ecological systems, permaculture, and holistic frameworks. (A summary of program elements is available in Table 3.3.)

Built out of these frameworks, students participate in learning activities that are hands-on and authentic. Students are encouraged think independently and critically, to discover and make connections, and to participate directly in their own learning. The curriculum fosters learning of material science, math, and social studies benchmarks through hands-on experiences: Students plan out their plots, dig in the dirt, foster plants' growth, and learn new recipes to cook the literal fruits of their labor (Williams \& Brown, 2011). These activities are tied to the real processes involved with growing and the land, necessitating acceptance of natural consequences that come externally via the seasons and internally via one's own efforts.

Unlike many hands-on classroom activities meant to be facsimiles of real-world activities, these activities exist in and shape the real world, and for this reason this type of gardening is intrinsically purposeful. The work involved in gardening serves a very concrete purpose in that students grow food which they are able to eat and take home to their families. Additionally, the garden embraces a multi-cultural perspective, discussing planting techniques and recipes from around the world. Students have the opportunity to utilize their particular cultural knowledge as strengths in the classroom, and form an explicitly rich and diverse learning community. At its core, the garden highlights an 
Table 3.3.

Overview of the Elements of the Learning Garden Laboratory

\begin{tabular}{|c|c|}
\hline Average dose & $6^{\text {th }}$ grade: 1.5 hrs per lesson, 1 time per week, 30 weeks. \\
\hline & $7^{\text {th }}$ grade: 1.5 hrs per lesson, 1 time per week, 15 weeks. \\
\hline \multirow[t]{3}{*}{$\begin{array}{l}\text { Conceptual } \\
\text { Framework }\end{array}$} & $\begin{array}{l}\text { Multi-level integrative developmental systems Model for Garden-Based Education } \\
\text { (Ratcliffe et al., 2010). }\end{array}$ \\
\hline & $\begin{array}{l}\text { Holistic integrated learning tied to the elemental experience of the natural } \\
\text { environment and growing things (Thorp, 2006). }\end{array}$ \\
\hline & $\begin{array}{l}\text { Intrinsic motivation as a source of energy and engagement in learning when } \\
\text { fundamental needs are met (Deci \& Ryan, 1985). }\end{array}$ \\
\hline \multirow{3}{*}{$\begin{array}{l}\text { Learning } \\
\text { objectives and } \\
\text { content }\end{array}$} & Coordinated with Science classes and grade-based Science concepts. \\
\hline & Incorporates Math, English, and Social Studies concepts. \\
\hline & $\begin{array}{l}\text { Based in systems thinking, ecological awareness, and an appreciation for } \\
\text { multicultural diversity. }\end{array}$ \\
\hline \multirow[t]{4}{*}{$\begin{array}{l}\text { Projected } \\
\text { Outcomes }\end{array}$} & $\begin{array}{l}\text { Engage students in gardening and Science learning, and promote academic } \\
\text { motivation, engagement, and resilience. }\end{array}$ \\
\hline & Improve learning and achievement. \\
\hline & $\begin{array}{l}\text { Instill a sense of purpose, belonging, community, self-efficacy, autonomy, and } \\
\text { ownership. }\end{array}$ \\
\hline & Promote ecological awareness and stewardship for the land. \\
\hline \multirow[t]{3}{*}{$\begin{array}{l}\text { Curricular } \\
\text { Principles }\end{array}$} & $\begin{array}{l}\text { Curricular learning environment: provide hands-on, project based, place-based } \\
\text { education engaging youth in authentic ongoing activities that integrate curricula } \\
\text { across disciplines, reinforcing concepts and abstract ideas (Ratcliffe et al., 2010). }\end{array}$ \\
\hline & $\begin{array}{l}\text { Physical learning environment: improve quality and diversity of learning } \\
\text { environments and safe spaces, including opportunities for visual learning and } \\
\text { direct experiences of abstract concepts (Ratcliffe et al., 2010). }\end{array}$ \\
\hline & $\begin{array}{l}\text { Social learning environment: foster caring relationships, provide high expectations } \\
\text { and clear feedback, and explain the relevance and importance of activities and } \\
\text { rules while soliciting input from students and listening to and respecting their } \\
\text { opinions (Deci \& Ryan, 1985). }\end{array}$ \\
\hline \multirow[t]{6}{*}{$\begin{array}{l}\text { Examples of } \\
\text { Gardening } \\
\text { activities }\end{array}$} & $\begin{array}{l}\text { Planning activities: Students diagram own 3' by 4' garden plot, including types of } \\
\text { plants. }\end{array}$ \\
\hline & Soil preparation activities: Students dig new beds and mix compost. \\
\hline & Planting activities: Students transplant seedlings. \\
\hline & Tending activities: Students water and weed beds. \\
\hline & Harvesting activities: Students pick and wash vegetables. \\
\hline & Preparing and eating activities: Students cook and eat vegetables. \\
\hline
\end{tabular}

Note. From Skinner, Chi, \& the LEAG, 2012. 
intimate understanding of ecological processes, cultivating an awareness of food systems, water cycles, and natural resources that can foster a sense of real stewardship for the earth. The gardening curriculum focuses on learning processes that highlight systemic inter-relationships, emergent properties, organic social interactions, uncertainty and creativity. Students learn thinking skills and content that allows them to consider complex world issues and think about solutions. These types of learning activities meet and encourage early adolescents' capacities and desires for purpose in their schoolwork. Moreover, they are an illustration of the types of hands-on, heads-on, contextually rooted and authentic academic experiences ideal for fostering engagement (Newman, 1992; Skinner, Chi, \& the LEAG, 2012). At the same time, a sense of purpose is not something that can be assigned to a group of students; students must really be involved in an energized, heads-on and hands-on way to reap the meaningful aspects of benefit of authentic activities. Taking into account the importance of engagement with purposeful activities for purpose to be possible, researchers of authentic and purposeful activities may wish to consider measuring students' engagement in those activities as well as, or in place, of measuring the nature of the activities themselves. 


\section{Research Questions and Hypotheses}

This study seeks to identify the developmental factors that might bolster motivational and academic resilience in at-risk eighth-grade students in a traditional middle school setting. To generate hypotheses, the study relies on a developmentallyadapted model of resilience that views the transitions to early adolescence and middle school as developmental risk factors, that considers student engagement along with achievement as markers of educational resilience, and that highlights enabling factors from self-determination theory, stage-environment fit theory, and positive psychology that may be especially salient at these transitions. To accomplish these goals, we must first understand what resilience means for this age group, and then we can consider developmentally salient factors that might support such resilience.

In the first step, this study will examine whether, in the context of the risks presented by demographic factors and middle school structures, eighth-graderness really does appear to pose an additional risk to motivation and achievement. In this step we will examine whether at-risk eighth-graders in a traditional middle school are significantly lower in engagement and GPA than sixth graders in the same context, and whether a developmentally calibrated strategy for forming resilience groups can be used. If they are, this would suggest that a developmentally calibrated strategy for examining resilience might be useful. If so, motivational, academic, and dual resilience groups are formed for sixth and eighth-grade students.

The next set of research questions investigates a set of enabling and protective developmentally-selected resources--specifically, autonomy, teacher support, peer 
support, and engagement in an authentic and purposeful academic work via a gardenbased education program. The second, third, and fourth research questions investigate whether students in motivational, academic, and dual motivational and academic resilience groups (respectively) differ based on their levels of autonomy, teacher support, peer support, and garden engagement. According to SDT, these factors should be supportive across the developmental spectrum. However, as discussed in Chapter 3, it is possible that selected aspects of a student's motivational process may become more important in early adolescence. Hence, this set of questions investigates whether those factors are more important to the resilience of eighth graders than that of sixth graders.

The fifth research question considers whether these four developmental resources predict dual resilience group membership. As with the previous set of questions, in order to see whether the unique effects are differentially developmentally salient, their power in predicting resilience will be contrasted between the eighth graders and the sixth graders. Finally, this study investigates whether autonomy serves as a mediator of the relationship between the other developmental resources and dual resilience group. In all, this study seeks to begin telling a story about the motivaiontal and academic resilience of at-risk eighth-grade students, establishing which developmentally salient aspects of students' motivational systems are important for these students.

\section{Research Question 1: Developmental Calibration of Resilience}

Do mean outcome levels and sample size indicate that use of a developmentally calibrated method for identifying resilience groups would be feasible and, if so, does 
that method produce adequate numbers of eighth-graders in each resilience group?

Research question 1a: Do eighth graders have significantly lower levels of engagement (motivation) and GPA (achievement) than sixth graders, indicating that "eighth-grader-ness may be a risk factor within this sample of demographically at-risk students in a traditional middle school?

Research question 1b: Resilience groups. Does the developmentallycalibrated method of forming eighth-grade resilience groups produce eighth-grade groups that are sufficiently large to proceed with analyses?

\section{Research Question 2: Motivational Resilience}

Do levels of the four developmental resources (autonomy, teacher support, peer support, and garden engagement) differ as a function of motivational resilience group?

Research question 2a: For eighth graders, do levels of the four developmental resources (autonomy, teacher support, peer support, and garden engagement) differ as a function of motivational resilience group?

Research question 2 b: Is the pattern of mean level differences in the four developmental resources as a function of motivational resilience group similar but more pronounced for eighth-grade students than for sixth-grade students?

Research question 2c: Are differences in mean levels of the developmental assets between motivational resilience groups greater for eighth graders than for sixth graders, as shown by a significant grade by motivational resilience group interaction term?

\section{Research Question 3: Academic resilience}


Do levels of the four developmental resources (autonomy, teacher support, peer support, and garden engagement) differ as a function of academic resilience group?

Research question 3a: For eighth-grade students, Do levels of the four developmental resources (autonomy, teacher support, peer support, and garden engagement) differ as a function of academic resilience group?

Research question 3b: Is the pattern of mean level differences in the four developmental resources as a function of academic resilience group similar but more pronounced for eighth-grade students than for sixth-grade students?

Research question 3c: Are differences in mean levels of the developmental assets between academic resilience groups greater for eighth graders than for sixth graders, as shown by a significant grade by motivational resilience group interaction term?

\section{Research Question 4: Dual resilience}

Do levels of the four developmental resources (autonomy, teacher support, peer support, and garden engagement) differ as a function of dual resilience group?

Research question 4a: For eighth-grade students, do levels of the four developmental resources (autonomy, teacher support, peer support, and garden engagement) differ as a function of dual resilience group?

Research question $4 \mathrm{~b}$ : Is the pattern of mean level differences in the four developmental resources as a function of dual resilience group similar but more pronounced for eighth-grade students than for sixth-grade students?

Research question 4c: Are differences in mean levels of the developmental 
assets between dual resilience groups greater for eighth graders than for sixth graders, as shown by a significant grade by motivational resilience group interaction term?

\section{Research Question 5: Predicting dual resilience}

Do each of the developmental resources predict dual resilience group membership?

Research Question 5a: Do each of the four developmental resources, with special focus on autonomy and garden engagement, uniquely predict dual resilience group membership for eighth-grade students when controlling for all other developmental resources?

Research question 5a1: Do each of the developmental resources, with special focus on autonomy and garden engagement, uniquely predict dual resilience group membership for eighth graders after controlling for all other developmental resources?

Research question 5a2: Are the four developmental resources descriptively stronger unique predictors of dual resilience group membership for eighth graders than for sixth graders?

Research question 5a3: Do the four developmental resources differ significantly as unique predictors of dual resilience group membership for eighth graders in comparison to sixth graders?

Research Question 5b: When tested separately, do each of the four developmental resources predict dual resilience group?

Research question 5b1: When tested separately, do each of the four developmental resources predict dual resilience group membership for eighth graders?

Research question 5b2: When tested separately are the four developmental 
resources descriptively stronger unique predictors of dual resilience group membership for eighth-graders than for sixth-graders?

Research Questions 5c: Does autonomy mediate the relationships between the three remaining developmental resources (teacher support, peer support, and garden engagement) and dual resilience group membership?

Research question 5c1: Does autonomy mediate the relationships between the three remaining developmental resources (teacher support, peer support, and garden engagement) and dual resilience group membership for eighth graders?

Research question 5c2: Is autonomy a descriptively stronger mediator of the relationships between the remaining developmental resources and dual resilience group for eighth graders than it is for sixth graders?

\section{Comparing Theoretical Approaches}

This study draws on both self-determination theory and stage-environment fit theory to investigate the nature of adolescent motivation and achievement. Whereas stage-environment fit theory posits that certain motivational needs become more important for students as they enter different developmental stages, self-determination theory posits that the motivational needs it defines are important for all students, regardless of their developmental period. With these research questions, we compare these two approaches, investigating the motivational needs within self-determination theory to see if, as proposed by stage-environment fit, some of them might be more important based on students' developmental progress (i.e., being in eighth vs. sixth grade), or if, as proposed by self-determination theory, these needs are equally 
Chapter 3: Purposes of Study

important for all students. 


\section{CHAPTER 4. \\ RESEARCH DESIGN AND METHOD \\ Sample}

Participants were 155 sixth-grade students, and 167 eighth-grade students from a middle school in a metropolitan area in the Pacific Northwest. Students ranged in age from $11-15($ Mean age $=12.72, S D=.93)$, and were 52\% female. Participants were largely low-income, and highly diverse in terms of both ethnicity and birth nationality: Seventy-five percent of students at the school qualified for free or reduced lunch and 41 percent spoke English as a second language. In this sample, 42.57 of students who reported their ethnicities identified as White, 26.24 percent identified as Latino/a, 18.37 percent identified as Asian, 8.16 percent identified as Black, and 4.66 percent identified as Native American. Of 457 total students, only students who had data for at least one of the outcome variables were included in this study, for a total sample of 322 students. Contributing to both the diversity and general low-income status of this school, it is situated in a very low-income neighborhood. This neighborhood, a central location for immigrant families, boasts a rich ethnic and national diversity, whose positive impacts can be seen in ethnic food and restaurant stores, community groups, and a qualitatively observed tendency for students to associate in cross-ethnicity peer groups. At the same time, the factors that make this neighborhood affordable for immigrant families bring a high-risk environment with limited resources. Linguistic barriers can make it difficult for young people to get proper access to resources. The neighborhood has high levels of food scarcity, and is in a classified "food desert" where access to 
healthy foods is limited. Crime rates are 1.5 to 3 times higher than the national average for all types of crime but murder. As is expected when high poverty is combined with multiple demographic risk factors, unemployment, violence, drug use, and incarceration are all factors in students' families (Escribano, 2010). For these reasons, we believe it is not unreasonable to classify the entire school as being at-risk.

The school is exceptional also, however, in its support from community partners. In collaboration with the graduate education program at a local state university, a sophisticated garden program offers students rich educational opportunities. In the year that these data were collected, all sixth and seventh graders and some eighth-grade students spent 1.5 hours once every week across the street at a 4 -acre garden site as a part of their science classes. Working from a curriculum rooted in sustainable, holistic, and ecological educational principles and rooted in a framework that celebrates multiculturalism, this garden incorporated state science and mathematics benchmarks into real-world, hands, on activities. Additionally, the garden directly aided families by giving students the opportunity to take produce home.

\section{Design}

This study uses a cross-sectional design, using the only data point at which all variables of interest are present in a larger study. Data for this study were collected in spring of one school year. Students completed surveys, which used a five-point Likerttype scale. Participants marked 1 for items that were "not at all true" and 5 for items that were "totally true". Scales included both negatively- and positively-worded items. Negatively-worded items were reverse-coded and averaged with positively worded 
items to produce scale scores. All resultant scales ranged from 1 to 5, with higher values indicating more of the respective construct. See Appendix A for a complete list of items.

\section{Measures}

\section{Profile of Engagement and Disaffection in School}

Eleven survey items assessed students' feelings of engagement, disaffection, and amotivation in school (Skinner, Kindermann, \& Furrer, 2008) Nine items assessed selfreports of behavioral (e.g. "I try hard to do well in school") and emotional ("When we work on something in class, I feel bored") engagement and disaffection in school. Two items assessed students' amotivation or lack of motivation in school, for example, "I really don't care about school." Reliability for a combined scale with all 11 had an alpha value of .87. Although engagement, disaffection, and amotivation are all distinct concepts, they are sufficiently inter-correlated and conceptually cohesive to permit their combination for use in quantifying a student's engagement profile.

\section{Teacher Support}

Nine items assessed student's perceptions of their teachers' provisions of motivational support (Skinner \& Belmont, 1993). Within those, three items assessed teacher's provision of structure ("I know what my teacher expects of me in class"), three items assessed teacher's provision of autonomy support ("My teachers don't give me much choice about how I do schoolwork", reverse-coded), and three items assessed teacher's provision of involvement (“Sometimes I wonder if my teachers really like me", reverse-coded). 


\section{Peer Support}

Twelve items assessed students' perceptions of positive peer relationships (Escribano, 2010; Skinner \& Escribano, 2010). Four items assessed perceptions of peer support, that is, that a student feels understood, respected, and liked by peers (e.g. "My friends respect and appreciate me".) Five items assessed peer belonging, that is, students feelings of membership and closeness with peers (e.g. "I feel like an outsider with my friends", reverse-coded). Three items assessed engagement with peers, a feeling of mutual caring, enjoyment, and satisfaction with peers (e.g. "My friends and I enjoy hanging out together".)

\section{Autonomy}

Students' perceptions of autonomy in school, the extent to which students did homework for reasons that were personally important to them, was assessed with four items from the identified self-regulation scale within a larger motivation scale (Ryan \& Connell, 1989). Items asked the question "Why do I do my home/school work," with students rating their agreement with items such as "because I want to understand the subject", and "because doing well in school is important to me."

\section{Profile of Engagement in Garden-based Education}

Sixteen items assessed students' ratings of engagement, disaffection, and amotivation in a garden-based education project (Skinner \& Chi, 2012). Three items assessed student's behavioral engagement (e.g. "When I'm in the garden, I listen carefully to our gardening teacher",) and three items assessed behavioral disaffection (e.g. "When I'm in the garden I just act like I'm working.") Four items assessed 
students emotional engagement (e.g. "When I'm in the garden, I feel good,"), and three items assessed emotional engagement (e.g. "When I'm in the garden, I am wet, dirty, not really interested in what we are doing.") Finally, three items assessed students' amotivation in the gardening program (e.g. "I don't care if I miss gardening class.")

\section{Achievement}

Students' grades in math, science, and social studies for the spring quarter were obtained from school records. Grades were converted to a standard four-point gradepoint average, where an A is a 4.0 and an $\mathrm{F}$ is 0 . Scores across subjects were averaged to give each student an achievement score. 


\section{CHAPTER 5.}

\section{RESULTS}

This study sought to begin an investigation of developmentally-calibrated resources for eighth graders' motivational and academic resilience. As a first step, lower mean levels of motivation and achievement for eighth-grade students suggested that "eighth-grader-ness" may indeed be a risk factor for motivational and academic performance, and this information guided the creation of developmentally-calibrated resilience groups. The following sections describe the dataset in general, the process of creating groups, testing whether those groups differed with respect to four developmentally-selected resources, and the exploration of those resources as predictors of group membership. We began investigating the data with a description of missing data, and a set of initial descriptive analyses to establish a foundational picture of the basic nature of this dataset. Exploration of internal consistencies, mean levels, and inter-correlations for scales used in the study permits a descriptive view into the trends explored in later analyses.

Because these analyses were meant to test a new way of investigating resilient processes in students, we used two parallel approaches to analyze patterns in the data. First, SPSS was used to form a clear picture of mean-level differences. In this first analysis phase, we first examined whether there are significant grade differences on which to base developmental calibration of resilience groups. We continue by forming the resilience groups and running a series of MANOVAs to investigate mean-level differences among groups. In this way, we intended to form an initial understanding of 
the relationship between motivational and academic resilience processes in middle school, and the developmental assets that may support them. In the second phase, we used Mplus (Muthen \& Muthen, 1998-2007) to formally model our preliminary working framework, using a series of regressions to test our four developmental resources as predictors of dual resilience group membership.

\section{Preliminary Analyses}

\section{Missing Data}

Meaningful missingness and sample characteristics. Incomplete data can be an especially complicated issue when researching risk and adaptation. Many procedures for replacing values are problematic in that they bias results and may reflect patterns that would not have existed if the original data were available. At the same time, deleting missing cases is yet more problematic, reducing sample size and generalizability to only those participants whose data are present. Some datasets have data missing at random (MAR), due to an unplanned absence or overlooked survey item. Occasional missing values can be a function of nothing more than the chance of data collection, reflecting data that are missing completely at random. For many other missing data situations, however, the fact that a student is missing data is meaningful information: data are not missing at random (NMAR) because their missingness is related to some real phenomenon. For example, if a child is never able to return a consent form from a parent, is chronically absent from school, encounters literacy difficulties, or is simply bored while taking the survey, that child's missing data may reflect an underlying problem relevant to the study. Such problems can be risk factors 
for students, meaning data may not generalize to the risk-affected students from whom researchers may be trying to learn.

Still, some estimation techniques for missing data (estimation maximization or EM is one such technique) have been shown to produce relatively unbiased estimates even when missingness seems to reflect some underlying meaning; that is, data are not missing completely at random (MCAR), but missingness is isolated enough that data can be still considered functionally missing at random (MAR). However, in other cases, missingness is not in error, but a reflection of reality--for example, eighth grader will be "missing" data for a class only offered to sixth-graders. In those situations, the researcher may not wish to estimate scores that do not reflect a genuine process. At the same time, increasingly sensitive estimation procedures can at time refine parameter estimates by considering any and all available information.

For this exploratory study, we intended to investigate the real processes that students have experienced in school, including those processes associated with risky situations that may prevent students from providing data. After initial data processing and cleaning, there were 367 sixth- and eighth-grade students in our dataset. We selected those students whose school records showed reasonable evidence of both being in school for the period during the study and who had some version of our outcome variables (grades and reports of engagement). The resulting sample comprised 322 students who had either school records of grades, their own self-reports of data, or both.

Missingness. For this resultant group of students, we used the Missing Values 
module in SPSS 17 to further investigate missing data pattern. Cases were analyzed on a case-wise and variable-wise basis, examining whether data could be considered missing at random (MAR), missing completely at random (MCAR), or not missing at random (NMAR). These analyses suggested that data were NMAR, with a significant result for Little's MCAR test $\left(X^{2}=56.71(33), p=.006\right)$ suggesting possible meaningful structural differences within the sample.

Proportions of missingness differed markedly in relation to grade level and scores on other study variables. Notably, approximately 65 eighth graders and 25 sixth graders lacked had GPA scores but lacked engagement, autonomy, teacher support, or peer support data. Additionally, approximately 40 eighth graders and 40 sixth graders appeared to not have participated in any garden-based activities. For the purposes of this study, we decided to cautiously proceed with including all students currently in the sample in order to better understand the processes in a wider variety of eighthgrade students.

Multiple imputation for Research Questions 1-4. The first phase of this study, which investigates mean levels of study constructs to create resilience groups and explore their relationships with developmental assets, was conducted in SPSS 17. The SPSS 17 missing values module was used to estimate missing values by creating five imputations of the original dataset. SPSS Multiple Imputation has the ability to pool estimates from each imputation, thus incorporating estimation error and preserving the more-robust nature of maintaining multiple separate imputations. However, pooled estimates are not currently offered for ANOVA $F$ tests, which are the 
central analyses for this section. To be able to perform the hypothesized tests of meanlevel differences, we used the mean of the five imputations, aggregating the five datasets into one. Initial descriptive statistics for each imputation indicated general consistency between imputations.

\section{Measurement Properties and Descriptive Statistics}

Initial descriptive analyses were conducted using SPSS 17, investigating each variable used in the study. With the exception of student GPA, all study constructs were aggregated from students' self-report item responses. Negatively-worded items were reverse-coded, and items were averaged to produce a single score. Students' values for these scales--that is, for engagement as well as for perceived autonomy, teacher support, peer support, and garden engagement-- could range from 1 to 5 , with higher scores indicating higher levels of the construct. GPA was a single score obtained from school records. Means, standard deviations, internal consistencies, item counts, and the range of values for each construct are listed in Table 5.1 and Table 5.2.

Statistics for entire sample. Initial investigation of scale statistics for all students showed generally satisfactory measurement properties, albeit with generally high mean levels (as discussed later). Internal consistencies for each construct were acceptable. Teacher support demonstrated satisfactory (.73) internal consistency. Cronbach's alpha for all other scales ranged from good (.84 for peer support) to excellent (.92 for garden engagement) values. 
Table 5.1

Descriptive Statistics and Scale Properties

\begin{tabular}{|c|c|c|c|c|c|c|}
\hline Construct & $\begin{array}{c}\text { Number } \\
\text { of } \\
\text { Items }\end{array}$ & $\alpha$ & $M$ & $(S D)$ & Min & $\operatorname{Max}$ \\
\hline \multicolumn{7}{|l|}{ Markers of Resilience } \\
\hline Engagement Profile & 11 & .86 & 4.00 & $(.61)$ & 1.18 & 5.00 \\
\hline Grade Point Average (GPA) & 1 & -- & 2.88 & $(.95)$ & 0.00 & 4.00 \\
\hline \multicolumn{7}{|l|}{ Developmental Resources } \\
\hline Autonomy & 4 & .88 & 3.75 & $(.96)$ & 1.00 & 5.00 \\
\hline Teacher Support & 9 & .74 & 4.01 & $(.57)$ & 1.44 & 5.00 \\
\hline Peer Support & 12 & .84 & 4.24 & $(.55)$ & 1.00 & 5.00 \\
\hline Garden Engagement & 16 & .92 & 3.59 & $(.77)$ & 1.00 & 5.00 \\
\hline
\end{tabular}

Standard deviations for each scale showed moderate variability in scores $(.55$ to .96). However, the generally high mean levels may be associated with potential ceiling effects or range restrictions in the data: both the engagement scale and teacher support scale showed moderate range restrictions, with no scale score reaching the minimum. While no variables demonstrated clear ceiling effects (as indicated with mean levels falling within one deviation of the maximum), the mean for peer support (4.24) falls only slightly below that threshold (maximum of 5.00 minus standard deviation of .55). Accordingly, all variables showed some amount of negative skew, but most were below values of 1 (skewness $=-.70$ to $-.86 .$, S.E. $=.14$ for all except peer support). Here again, the value for peer support was more problematic, with a skewness statistic of -1.41, S.E. $=.14$, indicating moderate but not unacceptable skew. Examination of kurtosis statistics for peer support revealed a high kurtosis statistic 
$(4.88$, S.E. $=.27)$, which indicates a generally flat or "high-shouldered" distribution.

Overall, it seems that generally uniform high levels of reported peer support may limit the ability to make inferences, but that all variables still offered some amount of meaningful variability.

Statistics by grade level. This study centers on developmental changes in motivation across middle school, investigating these changes using differences in motivation between sixth- and eighth-grade students. Table 5.2 displays descriptive statistics and measurement properties for the same dataset split into sixth- and eighthgrade groups. Measurement properties seemed similar across grades, although reliabilities were slightly lower in sixth-graders for teacher support ( $\operatorname{sixth}$ grade $\alpha=$ .70 , eighth grade $\alpha=.79$ ) and peer support ( $\operatorname{sixth}$ grade $\alpha=.83$, eighth grade $\alpha=.91$ ). Inspection of mean levels showed that eighth graders' scores for most constructs were descriptively somewhat lower (by approximately $1 / 3 \mathrm{SD}$ ), than those for sixth graders, with GPA showing the greatest difference in both mean levels and variances $(\mathrm{M}=2.61$, $S D=1.05$ for eighth grade, $\mathrm{M}=3.18, S D=.73$ for sixth grade). However, eighth-grade peer support scores $(\mathrm{M}=4.26, S D=.51)$ appeared to be no lower than those for sixth graders $(\mathrm{M}=4.23, S D=.60)$. Other than eighth-grade GPA, autonomy showed the highest variation of scores within both grades $(S D=.94$ eighth grade, .96 sixth grade; all other construct SDs $=.51$ to.76). Although later analyses formally tested potential differences between grade groups, these results suggested that eighth-grade students did have generally lower scores than sixth-grade students. 
Table 5.2

Table Summary of Descriptive Statistics and Measurement Properties for Sixth-and Eighth-grade Students

\begin{tabular}{|c|c|c|c|c|c|c|c|c|c|c|c|}
\hline \multirow{2}{*}{ Construct } & \multirow{2}{*}{$\begin{array}{l}\text { Number } \\
\text { of Items }\end{array}$} & \multicolumn{5}{|c|}{ Eighth-Grade Students } & \multicolumn{5}{|c|}{ Sixth-Grade Students } \\
\hline & & $\alpha$ & $M$ & $(S D)$ & Min & Max & $\alpha$ & $M$ & $(S D)$ & Min & Max \\
\hline \multicolumn{12}{|l|}{ Markers of Resilience } \\
\hline Engagement Profile & 11 & .85 & 3.91 & $(.57)$ & 1.91 & 5.00 & .88 & 4.10 & $(.64)$ & 1.18 & 5.00 \\
\hline Grade Point Average (GPA) & 1 & -- & 2.61 & $(1.05)$ & 0.00 & 4.00 & -- & 3.18 & $(.73)$ & 0.60 & 4.00 \\
\hline \multicolumn{12}{|l|}{ Developmental Resources } \\
\hline Autonomy & 4 & .88 & 3.62 & $(.94)$ & 1.00 & 5.00 & .87 & 3.89 & $(.96)$ & 1.00 & 5.00 \\
\hline Teacher Support & 9 & .79 & 3.96 & $(.54)$ & 1.44 & 5.00 & .70 & 4.06 & $(.59)$ & 1.78 & 5.00 \\
\hline Peer Support & 12 & .91 & 4.26 & $(.51)$ & 1.00 & 5.00 & .83 & 4.23 & $(.60)$ & 1.67 & 5.00 \\
\hline Garden Engagement & 16 & .91 & 3.35 & $(.76)$ & 1.00 & 4.88 & .90 & 3.85 & $(.70)$ & 1.00 & 5.00 \\
\hline
\end{tabular}

Note. Total $\mathrm{N}=322$. Students' combined G.P.A scores were obtained from school records and could range from $0-4$. All other scales were student-report and scores could range from 1-5. Higher scores indicate higher levels of the respective construct. 
Skew and kurtosis statistics suggested that some of the potential deviations from normal distributions observed at this point may be due to distribution differences between grades. In one notable example, the kurtosis statistic for sixth graders' ratings of peer support was a moderate $2.35($ S.E. $=.39)$, while that for eighth graders was a strong $8.97($ S.E. $=.37)$. Sixth-grade data also showed moderate kurtosis in engagement and garden engagement scores (kurtosis $=2.56$ and 2.30, respectively, S.E. $=.39$ ), again indicating a somewhat flat distribution of these scores. Along with what seem to be generally high mean levels, all sixth-grade levels showed some negative skew, (skewness $=-.83$ to -1.16 for all constructs, S.E. $=.19$ ), and range restrictions in that only autonomy and garden engagement reached the lowest scores. No construct demonstrated clear ceiling effects (via the construct mean falling within one standard deviation of the maximum possible score), but all were close (seeTable 5.1), with no mean level falling two standard deviations below the maximum. Thus, sixth-grade scores seem to be generally high, with constructs trending towards ceiling effects.

In comparison, eighth-grade scores were slightly lower, and generally showed less restriction of variance. Only teacher support and peer support showed evidence of non-normal distributions ( skewness $=-.92$ and -1.75, S.E. $=.19 ;$ kurtosis $=2.84 \&$ 8.97 , S.E. $=.37)$. The mean for peer support $(4.26, S D=.51)$, approached the maximum score of 5. Thus, preliminary patterns at this point could be interpreted such that eighth graders differed from sixth graders by having generally lower mean levels and more-normally-distributed scores. Preliminary variable-wise patterns also 
emerged across grades, such that both eighth-grade and sixth-grade ratings of peer support showed the same high mean levels and limited distributions, and autonomy scores showed wider distribution in their slightly higher standard deviations. These potential trends were only descriptive observations, but offered what may be a helpful lens for future findings or complications.

\section{Intra-construct Correlations}

Correlations between all study variables are presented in Table 5.3 (entire sample) and Table 5.4 (split by grade). In most circumstances, the two variables marking resilience (engagement and GPA, respectively) were positively associated with the four hypothesized developmental assets (autonomy, teacher support, peer support, and garden engagement. Engagement and GPA were moderately intercorrelated, and the four developmental assets were also generally positively intercorrelated with one another. All significant correlations were positive.

Correlations for entire sample. In analyses of the entire sample, most study variables were significantly inter-correlated with the other variables, as expected. All significant correlations were positive. Engagement (marking motivational resilience) and GPA (marking academic resilience) were correlated at $r=.34, p<.001$. The moderate strength of this relationship indicated that these variables reflected related but distinct processes, and suggested that resilience groups based on these variables may have also reflected separate but complementary aspects of students' academic success.

Each of the four developmental asset variables was positively and significantly 
related to the others. Correlations among students' perceptions of autonomy, teacher support, peer support, and garden engagement were small to moderate ( $r \mathrm{~s}=.15$ to .52 , $p<.01)$. Of the developmental assets, perceptions of teacher support and autonomy support had the strongest connection $(r=.52, p>.001)$. The weakest relationships were between peer support and autonomy $(r=.15, p>.01)$, and peer support and teacher support $(r=.15, p>.01)$. Peer support generally showed the weakest correlations among the study variables. This may reflect a lack of meaningful variance within the peer support construct, which would be consistent with the possibly restricted distribution seen in the descriptive statistics.

Table 5.3

Inter-correlations among Study Constructs

\begin{tabular}{lllllll}
\hline & 1.Eng. & 2.GPA & 3.Aut. & 4.Tch. & 5.Peer & 6.Gard. \\
\hline Markers of Resilience & & & & & & \\
$\quad$ 1. Engagement & -- & -- & -- & -- & -- & -- \\
2. GPA & .34 & -- & -- & -- & -- & -- \\
$\quad \begin{array}{l}\text { Developmental Resources } \\
\text { 3. Autonomy }\end{array}$ & .70 & .26 & -- & -- & -- & -- \\
$\quad$ 4. Teacher Support & .62 & .26 & .52 & -- & -- & -- \\
5. Peer Support & .21 & $.00^{(\text {n.s. })}$ & $.15^{* *}$ & .32 & -- & -- \\
$\quad$ 6. Garden Engagement & .31 & .29 & .25 & .28 & $.15^{* *}$ & -- \\
\hline Note: All correlations significant at $p<.001$ unless indicated; ${ }^{\dagger} p<.10, * p<.05, * * p<.01$. \\
$N=322$.
\end{tabular}


The most important co-relationships for later analyses were those between the four developmental assets and the two resilience variables. Engagement showed positive associations with all four developmental assets, and strong relationships with both autonomy and teacher support $(r s=.62$ and .70 , respectively, $p<.001)$. These associations approached those suitable for internal consistencies and indicated that the teacher support and autonomy constructs may each share some underlying component with the engagement construct. GPA showed generally moderate relationships with all study variables $(r \mathrm{~s}=.26$ to $.29, p<.001)$ except peer support, which was not significantly correlated with GPA. When compared with engagement, GPA showed weaker relationships with all variables except garden engagement, which showed a similar relationship with GPA $(r=.29, p<.001)$ as with Engagement $(r=.31, p<.001)$. Of the four developmental asset variables, peer support showed the weakest relationships with the resilience group variables, with no significant relationship with $\operatorname{GPA}(r=.00, \mathrm{n} . \mathrm{s}$,$) and only a weak relationship (r=.21, p>.01)$ with engagement.

From these initial correlations, there seems to be support for the hypothesized relationships between all four developmental assets and motivational resilience (based on engagement) with autonomy support and teacher support showing the strongest relationships. Academic resilience (based on GPA) may show generally weaker relationships than motivational resilience, and initially shows no relationship with peer support. Engagement and GPA are correlated moderately, so their combination in dual resilience may show different patterns of relationships than either motivational or 
Table 5.4

Inter-Correlations for Eighth- and Sixth-grade Students.

\begin{tabular}{|c|c|c|c|c|c|c|}
\hline Eighth-grade Students $(n=167)$ & 1 & 2 & 3 & 4 & 5 & 6 \\
\hline \multicolumn{7}{|l|}{ Markers of Resilience } \\
\hline 1. Engagement Profile & -- & -- & -- & -- & -- & -- \\
\hline 2. GPA & .29 & -- & -- & -- & -- & -- \\
\hline \multicolumn{7}{|l|}{ Developmental Resources } \\
\hline 3. Autonomy & .67 & $.21^{* *}$ & -- & -- & -- & -- \\
\hline 4. Teacher Support & .58 & $.24^{* *}$ & .39 & -- & -- & -- \\
\hline 5. Peer Support & .33 & $.07^{(\text {n.s. })}$ & $.20^{*}$ & .41 & -- & -- \\
\hline 6. Garden Engagement & $.16^{*}$ & $.20^{* *}$ & $.10^{\text {(n.s.) }}$ & $.19^{*}$ & $.10^{\text {(n.s.) }}$ & -- \\
\hline Sixth-grade Students $(n=155)$ & 1 & 2 & 3 & 4 & 5 & 6 \\
\hline \multicolumn{7}{|l|}{ Markers of Resilience } \\
\hline 1. Engagement Profile & -- & -- & -- & -- & -- & -- \\
\hline 2. GPA & .38 & -- & -- & -- & -- & -- \\
\hline \multicolumn{7}{|l|}{ Developmental Resources } \\
\hline 3. Autonomy & .70 & $.26^{* *}$ & -- & -- & -- & -- \\
\hline 4. Teacher Support & .65 & .27 & .64 & -- & -- & -- \\
\hline 5. Peer Support & $.12^{\text {(n.s.) }}$ & $-.08^{\text {(n.s.) }}$ & $.11^{\text {(n.s.) }}$ & $.24^{* *}$ & -- & -- \\
\hline 6. Garden Engagement & .41 & $.25^{* *}$ & .37 & .34 & $.24^{* *}$ & -- \\
\hline
\end{tabular}


academic resilience in isolation.

Correlations by grade. An investigation of correlations when sixth graders and eighth graders were analyzed separately revealed generally similar patterns between grades. As in the analyses of all students together, analyses within separate grades found that engagement and GPA were moderately correlated, but appeared to share a stronger relationship for sixth-graders than for eighth-graders $(r \mathrm{~s}=.38$ and .29 , respectively, $p>.001)$. Engagement showed descriptively stronger relationships with many variables than did GPA. Autonomy and teacher support maintained strong relationships with engagement and weak relationships with GPA. Several apparent grade differences emerged when examining perceptions of garden engagement and peer support (consistent with trends in descriptive statistics and measurement properties). Notably, peer support remained unrelated to GPA for both grades, but diverged sharply in regard to its relationship with engagement for different grades $(r=$ $.33, p>.001$ for eighth grade; $r=-.08$, n.s. for sixth grade) which may lend support to an increase in the salience of peers for eighth graders. In contrast, sixth graders seemed to show a stronger relationship between garden engagement and overall engagement $(r=.41, p>.001)$ than did eighth graders $(r=.16, p>.05)$.

When looking at patterns of relationships within the developmental assets, teacher support appeared to have a stronger association for sixth graders $(r=.64,001)$ than eighth-graders, $(r=.39, p<.001)$. Peer support was associated with teacher support for eighth graders $(r=.20, p<.05)$, but not sixth graders $(r=.11$, n.s. $)$. Garden engagement was associated with autonomy and peer support for sixth graders $(r=.37$, 
$p<.001$ and $r=.24, p<.01$, respectively), but not for eighth graders $(r \mathrm{~s}=.10$ and .10 , n.s.). Thus, when comparing grades, the general tendency for engagement to have stronger relationships with developmental assets than GPA remained consistent. The developmental assets themselves, however seemed to show somewhat differing patterns for sixth- and eighth-graders, especially peer support and garden engagement.

An investigation of correlations within sixth- and eighth-grade students separately revealed that the two resilience marker variables, engagement and GPA, had a relatively similar pattern of relationships for both grades. GPA shared a moderate association with engagement, as well as with autonomy and teacher support. Engagement showed a strong relationship with autonomy and teacher support. Garden support had a weak relationship with both markers except for sixth-grade engagement, which showed a moderate-to-strong relationship. Of the two markers across the two grades, peer support showed no relationships with the resilience markers except for eighth-grade engagement.

\section{Summary of Preliminary Analyses}

Engagement and GPA, the two variables used as markers of resilient (vs. stress-affected) processes, do appear to be positively associated with levels of the four variables hypothesized to serve as development assets (vs. liabilities). This indicates that those four variables - i.e. students' perceptions of autonomy over schoolwork, teacher support, peer support, and engagement in a gardening class — may be helpful in understanding what differentiates resilient from stress-affected students. Moreover, engagement and GPA seem to show different patterns, both in their own mean levels 
and in their relationships to levels of asset variables, such that engagement has a more consistent and stronger relationships with other variables than GPA. This suggests that separate examinations of motivation (via engagement), achievement (via GPA), and the overlap between the two may be useful in showing differential patterns for each of these categories of academic success.

Patterns did differ by grade, descriptively. Eighth-graders seemed to have lower mean levels on almost all variables, and relationships between the resilience variables and four asset variables seemed to differ somewhat between grade levels. This provides initial support for the utility provided by developmentally calibrating and understanding of academic outcomes and supporting processes. Peer support and garden engagement showed the most pronounced differences between grades, but also demonstrated the most problematic measurement properties. Potential ceiling effects and a large proportion of missing data, respectively, indicate that analyses of these variables should be interpreted with caution.

Although these initial results do not test concrete between variables or grades, but show that the four hypothesized developmental assets do show general positive associations with the two resilience markers. Additionally, descriptive differences in patterns between and among constructs for the two grade levels suggest that the hypothesized grade differences in motivational resilience may be supported in differential relationships and processes across grades, in addition to different mean levels. 


\section{Research Question 1: “Eighth-gradeness” and Resilience Groups}

Do mean outcome levels and sample size indicate that use of a developmentally calibrated method for identifying resilience groups would be feasible and, if so, does that method produce adequate numbers of eighth graders in each resilience group? Research question 1a: Do eighth graders have significantly lower levels of engagement (motivation) and GPA (achievement) than sixth graders, indicating that "eighth-grader-ness may be a risk factor within this sample of demographically atrisk students in a traditional middle school?

Resilient processes require both a) risk and b) competent adaptation in spite of that risk (Masten, 2001). When considering the nature of eighth-graders' resilient motivational and academic processes, resilient development in spite of "eighth-graderness" requires, by this definition, that eighth-grader-ness indeed appears to be a risk factor. To investigate this hypothesis, a one-way ANOVA tested mean levels of GPA and engagement across grade levels (see Table 5.5).

As hypothesized, eighth-grade students' mean levels of engagement were significantly lower than those of sixth-grade students, and eighth-graders' GPA scores were significantly lower than those of sixth-graders. These results indicate that, in this sample, eighth-grade students' levels of motivation and achievement were lower than those for sixth-grade students who experience the same risk factors posed by demographics and being in a traditional middle school. This, in turn, suggests that it is possible that something about being an eighth grader poses an additional risk factor in the context of these other risk factors. To investigate the potential impacts of the risk 
that eighth-grader-ness seems to pose, it may be appropriate to use a developmentally calibrated method for selecting resilience groups.

Table 5.5

Mean Levels of Motivation and Achievement by Grade and Gender.

\begin{tabular}{|c|c|c|c|c|c|c|}
\hline \multirow{3}{*}{$\begin{array}{l}\text { Outcome } \\
\text { Variable }\end{array}$} & \multicolumn{6}{|c|}{ Mean Levels } \\
\hline & \multicolumn{2}{|c|}{$\begin{array}{l}8^{\text {th }} \text { Grade } \\
(n=167)\end{array}$} & \multicolumn{2}{|c|}{$\begin{array}{l}6^{\text {th }} \text { Grade } \\
(n=155)\end{array}$} & & \\
\hline & Mean & (SD) & Mean & (SD) & & \\
\hline Engagement & 3.91 & $(.60)$ & 4.10 & $(.64)$ & & \\
\hline \multirow[t]{3}{*}{$G P A$} & 2.61 & $(1.05)$ & 3.18 & $(.73)$ & & \\
\hline & \multicolumn{2}{|c|}{$\begin{array}{c}\text { Male } \\
(n=167)\end{array}$} & \multicolumn{2}{|c|}{$\begin{array}{c}\text { Female } \\
(n=155)\end{array}$} & & \\
\hline & Mean & (SD) & Mean & (SD) & & \\
\hline Engagement & 3.90 & $(.63)$ & 4.11 & $(.58)$ & & \\
\hline \multirow[t]{3}{*}{$G P A$} & 2.79 & $(.92)$ & 2.98 & $(.85)$ & & \\
\hline & \multicolumn{2}{|c|}{$\begin{array}{c}8^{\text {th }} \text { Grade } \\
\text { Male } \\
(n=88)\end{array}$} & \multicolumn{2}{|c|}{$\begin{array}{c}8^{\text {th }} \text { Grade } \\
\text { Female } \\
(n=79)\end{array}$} & $\begin{array}{c}6^{\text {th }} \text { Grade } \\
\text { Male } \\
(n=79)\end{array}$ & $\begin{array}{c}6^{\text {th }} \text { Grade } \\
\text { Female } \\
(n=76)\end{array}$ \\
\hline & Mean & (SD) & Mean & (SD) & Mean (SD) & Mean (SD) \\
\hline Engagement & 3.80 & $(.58)$ & 4.04 & $(.53)$ & 4.00 & $(.61)$ \\
\hline$G P A$ & 2.58 & $(1.01)$ & 2.64 & $(1.10)$ & 3.02 & $(.68)$ \\
\hline
\end{tabular}

Gender as a potential covariate. We planned to use gender as a covariate to control for the expected additional effect of gender on study variables, with girls' levels on all constructs expected to be higher than boys'. Descriptively, girls' mean levels of engagement and GPA were higher than boys' for both sixth and eighth graders, as was expected. Mean levels are reported in Table 5.5. In initial tests with 
engagement and GPA, gender appeared to show no problems with heterogeneity of variance, and so it was decided that gender could be included as a covariate in the subsequent analyses.

Research question 1b: Does the developmentally-calibrated method of forming eighth-grade resilience groups produce eighth-grade groups that are sufficiently large to proceed with analyses? Because it seemed that eighth-grader-ness poses an additional risk factor in this sample, on top of the risks posed by demographic status and being in a traditional middle school, a developmentally calibrated method was examined as a strategy to identify resilience groups.

Statistical considerations for forming groups. A series of power analyses were used to understand the extent to which dividing students into groups might limit the statistical power to reject the null hypotheses in the following research questions. In order to determine the extent to which group resilient group sizes could be unequal (i.e., by having fewer eighth-grade resilient group members and more eighth-grade stress-affected or average group members) and still detect effects in the analyses for the study, we calculated the effective sample size that corresponded to a range of possible configurations of group sizes. The program G*power (Faul, Erdfelder, Lang, \& Buchner, 2007) was then used to calculate the effect sizes detectible based on these effective sample sizes. We found the smallest possible cell sizes would prevent the detection of very small effects, but still had the power to detect large effects that might reasonably be expected given the high correlations between some of the study variables. Thus, we will proceed with the planned analyses. 
Steps for forming developmentally-calibrated resilience groups. The

following sequence of steps was taken to identify motivational resilience groups within sixth graders which then informed groups within eighth graders, followed by academic resilience groups within sixth- and then eighth-graders, and then dual resilience groups within sixth and eighth graders. The range of scores and group sizes for each resilience group are listed in Table 5.6. As the first step in forming motivational resilience groups, sixth-grade resilience groups were formed. Sixthgraders with the top third of engagement scores $(n=51)$ were used to form the motivationally resilient group. These were students whose levels of engagement were above 4.55. The lowest third of sixth graders $(n=51)$ had engagement scores below 3.89, and formed the motivationally stress-affected group. The remaining sixth-grade students $(n=53)$ formed the motivationally average group.

Second, these cut points were used to establish eighth-grade motivational resilience groups. Eighth-grade students with engagement scores above $4.55(n=28)$ formed the motivationally resilient group. Eighth-graders with engagement scores below $3.89(n=84)$ formed the motivationally stress-affected group. The remaining eighth graders $(n=55)$ formed the motivationally average group.

The same two-step process was used to establish academic resilience groups for sixth graders and then eighth graders. Sixth graders in the top third of GPA scores (at or above 3.67) formed the academically resilient group $(n=47)$, those in the bottom third of scores (2.80 and below) formed the academically stress-affected group $(n=50)$, and the remainder formed the academically average group $(n=58)$. Next, 
these cut points were used to identify the eighth-grade academic resilience groups.

Eighth graders whose GPA was above 3.60 formed the academically resilient group ( $n$

Table 5.6: Resilience Group Sizes and Range of Scores

\begin{tabular}{|c|c|c|c|c|}
\hline \multirow{2}{*}{\multicolumn{2}{|c|}{ Type of Resilience }} & \multirow{2}{*}{$\begin{array}{c}\text { Stress-Affected } \\
\begin{array}{c}\text { Group Size } \\
(\text { Range })\end{array}\end{array}$} & \multirow{2}{*}{$\begin{array}{c}\text { Average } \\
\text { Group Size } \\
(\text { Range })\end{array}$} & \multirow{2}{*}{$\begin{array}{c}\text { Resilient } \\
\text { Group Size } \\
(\text { Range })\end{array}$} \\
\hline & & & & \\
\hline \multirow[t]{3}{*}{$\begin{array}{l}6^{\text {th }} \text { Grade } \\
(n=155)\end{array}$} & $\begin{array}{c}\text { Motivation } \\
\text { (Engagement) }\end{array}$ & $\begin{array}{c}\mathbf{5 1} \\
(1.18-3.89)\end{array}$ & $\begin{array}{c}\mathbf{5 3} \\
(3.89-4.50)\end{array}$ & $\begin{array}{c}\mathbf{5 1} \\
(4.55-5.00)\end{array}$ \\
\hline & $\begin{array}{c}\text { Academic } \\
\qquad(G P A)\end{array}$ & $\begin{array}{c}\mathbf{5 0} \\
(0.60-2.80)\end{array}$ & $\begin{array}{c}\mathbf{5 8} \\
(3.00-3.60)\end{array}$ & $\begin{array}{c}\mathbf{4 7} \\
(3.67-4.00)\end{array}$ \\
\hline & $\begin{array}{c}\text { Dual }^{*} \\
(\text { Engagement }) \\
(G P A)\end{array}$ & $\begin{array}{c}\mathbf{2 4} \\
(1.18-3.89) \\
(.60-2.80)\end{array}$ & $\begin{array}{c}109 \\
(2.30-5.00) \\
(1.60-4.00)\end{array}$ & $\begin{array}{c}22 \\
(4.55-5.00) \\
(3.80-4.00) \\
\end{array}$ \\
\hline \multirow[t]{3}{*}{$\begin{array}{l}8^{\text {th }} \text { Grade } \\
(n=167)\end{array}$} & $\begin{array}{l}\text { Motivation } \\
\text { (Engagement) }\end{array}$ & $\begin{array}{c}\mathbf{8 4} \\
(1.91-3.88)\end{array}$ & $\begin{array}{c}\mathbf{5 5} \\
(3.90-4.45)\end{array}$ & $\begin{array}{c}\mathbf{2 8} \\
(4.55-5.00)\end{array}$ \\
\hline & $\begin{array}{c}\text { Academic } \\
(G P A)\end{array}$ & $\begin{array}{c}\mathbf{9 0} \\
(0.00-2.83)\end{array}$ & $\begin{array}{c}\mathbf{4 1} \\
(3.00-3.50)\end{array}$ & $\begin{array}{c}\mathbf{3 6} \\
\square 3.67-4.00)\end{array}$ \\
\hline & $\begin{array}{c}\text { Dual }^{*} \\
\text { (Engagement) } \\
(G P A)\end{array}$ & $\begin{array}{c}\mathbf{5 3} \\
(1.91-3.88) \\
(.00-2.83)\end{array}$ & $\begin{array}{c}101 \\
(2.64-5.00) \\
(.17-4.00)\end{array}$ & $\begin{array}{c}\mathbf{1 3} \\
(4.55-4.95) \\
(3.67-4.00)\end{array}$ \\
\hline
\end{tabular}

Note. Engagement could range from 1-5, and GPA could range from 0-4, with higher numbers reflecting higher levels of motivation and achievement.

* Dually group members consisted of students in both the motivational and academic resilience group. Dually stress-affected group-members consisted of students in both the motivational and academic stress-affected groups. All other students made up the Dually average group. 
$=28)$, those with GPAs below 3.00 formed the academically stress-affected group $(n=$ $90)$, and the remainder formed the academically average group $(n=41)$.

Finally, for both grades, students who showed both motivational and academic resilience formed dually resilient groups, while students who showed both motivational and academic stress-affectedness formed dually stress-affected groups. Sixth-graders with both engagement scores above 4.46 and GPAs above 3.60 formed the dual resilience group for sixth-graders $(n=22)$. Those with both engagement lower than 4.00 and GPA lower than 3.00 formed the sixth-grade dually stress-affected group $(n=22)$, and the remainder formed the dually average group $(n=114)$.

Similarly, eighth-graders with engagement higher than 4.46 and GPA higher than 3.60 formed the eighth-grade dual resilience group $(n=13)$. Those with engagement lower than 4.00 and GPA lower than 3.00 formed the dually stress-affected group for eighthgraders $(n=53)$, and the remainder formed the eighth-grade dually average group $(n=$ 101).

Despite lower mean levels, eighth-grade engagement and GPA scores were high enough that the same cut points as sixth-grade groups could be used and there were still eighth-graders at the resilient-group level of motivation, academic achievement, and the combination of the two. Small, unequal cell sizes can be problematic during analyses, lowering statistical power and complicating the calculation of marginal means. Accordingly, in the analyses using these groups, cell size was taken into consideration when interpreting results, and, whenever possible, steps were taken to make tests more robust against violations of assumptions. 


\section{Research Questions 2 - 4 ANOVAs: Resilience Group and Developmental Resource Levels for Eighth- vs. Sixth-Grade Students}

These three questions investigated the extent to which resilience group status predicted differences in eighth-graders' (vs. sixth-graders') mean levels of the four potential developmental resources. Questions 2, 3, and 4 investigated motivational, academic, and dual resilience, respectively, as the central IV in each series of analyses. These three versions of resilience groups all had three levels representing stressaffected vs. average vs. resilient processes. The four developmental resources (autonomy, teacher support, peer support, and garden engagement) were DVs in all analyses.

Within questions 2, 3, and 4, the same series of three sub-questions investigated the patterns of developmental resources for eighth graders as compared to sixth graders. Subquestions A investigated whether the levels of each of the four developmental resources differed as a function of resilience group for eighth-grade students. Subquestions B investigated whether levels of the resources differed as a function of resilience group for sixth graders in order to descriptively assess differences between the grades. Subquestions C investigated resilience group differences in the entire sample of sixth- and eighth-grade students, explicitly testing grade differences by including a resilience group by grade interaction term. We hypothesized that levels of teacher support, peer support, autonomy, and garden engagement would be significantly higher for students in motivationally resilient groups than those in motivationally average and stress-affected groups, and that these 
differences would be more pronounced for eighth-grade students than for sixth-grade students.

Prior to each subquestion, preliminary tests determined the appropriateness of including gender as a covariate. Gender was included as a covariate in the following analyses unless a) there was no main effect of gender on the DVs, in which case gender was omitted from the model, or b) there was evidence of a gender-by-group interaction, which would indicate a violation of the assumption of homogeneity of regression slopes. In the latter case, gender was included as an IV in order to assess the effect of gender on the DVs directly. Analyses used the sequential sum of squares method (Type I in SPSS) to correct for unequal cell sizes, and report Pillai's trace to provide additional robustness against the heterogeneity of covariance-variance matrices that accompanies unequal cell sizes.

\section{Research Question 2: Motivational Resilience}

Do levels of the four developmental resources (autonomy, teacher support, peer support, and garden engagement) differ as a function of motivational resilience group?

Research question 2a: for eighth graders? A 2x3 factorial MANOVA was used to investigate whether levels of the four developmental resources (autonomy, teacher support, peer support, and garden engagement; the four dependent variables) differed as a function of motivational resilience group for eighth-grade students. The independent variables were motivational resilience group (stress-affected vs. average vs. resilient) and gender (female vs. male). Gender was entered first in the model, 
followed by motivational resilience group. Mean levels of the four developmental resources as a function of gender and motivational resilience group and post-hoc test results are presented in Appendix B. Mean-level differences are represented in Figure 5.1.

Resilience group differences. In the omnibus model, there was a significant effect of motivational resilience group on the linear combination of the four developmental resources, Pillai's Trace $=.38, F(8,318)=9.39, p<.001$, partial $\eta^{2}=$ .19. Follow-up ANOVAs revealed significant main effects of motivational resilience group on all four individual developmental resources. Post-hoc tests showed that all four resources (autonomy, teacher support, peer support, and garden engagement) served as general motivational resources in that their levels were significantly higher in the resilient group than in the stress-affected group. Autonomy, teacher support, and (marginally) garden engagement all seemed to help enable students' motivational resilience, as shown by having higher levels in the resilient group than in the average group. Autonomy and (marginally) teacher support both seemed to help protect against motivational stress-affectedness, as shown by having higher levels in the average group than in the stress-affected group.

There was also significant effect of gender on the DVs, Pillai's Trace $=.11$, $F(4,158)=4.68, p=.001$, partial $\eta^{2}=.11$. Follow-up ANOVAS showed effects for autonomy, peer support, and (marginally) teacher support. These effects were such that female students' levels of these resources were higher than those for male students.

\section{Research question $2 \mathrm{~b}$ : more saliently for sixth graders than eighth}


Figure 5.1. Mean Levels of Autonomy, Teacher Support, Peer Support, and Garden Engagement in Eighth-and Sixth-grade Motivational Resilience Groups.

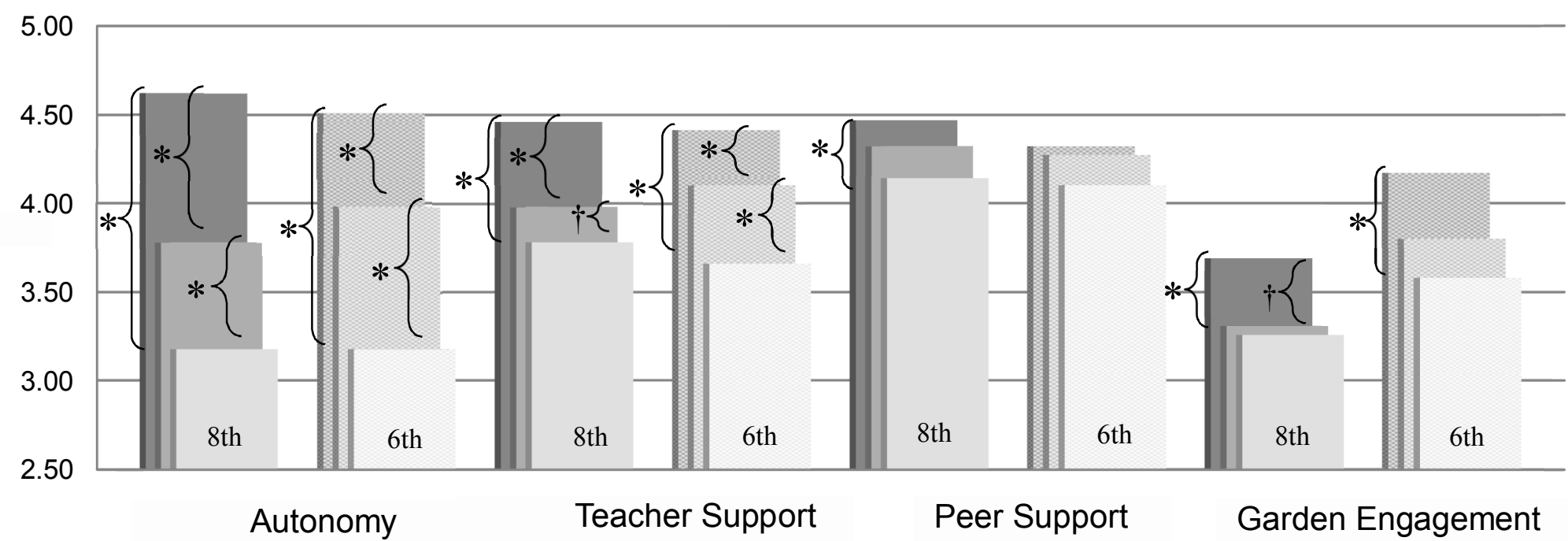

Note: Total $n=322$. Eighth grade $n=167$, sixth grade $n=155$. Bars show mean levels of autonomy, teacher support, peer support, and garden engagement in eighth- and sixth-grade motivational resilience groups. Mean levels of each developmental resource for each grade are clumped, with resilient groups in rear and stress-affected groups in front. Significant differences between groups shown by bracket and $*$ for $p<.05$ and $\uparrow$ for $p<.10$. 
graders? In order to see whether the pattern of mean-level differences in the developmental resources appeared to be more pronounced for eighth graders, a sixthgrade model was compared to that for eighth graders. A one-way MANOVA tested whether sixth-grade levels of the four DVs (autonomy, teacher support, peer support, and garden engagement) differed as a function of the IV, motivationally resilient vs. average vs. stress-affected group membership. In general, the sixth-grade pattern of mean-level differences did not differ greatly from those shown in the eighth-grade model. Mean levels and post-hoc test results can be can be found in Appendix B, and mean-level differences are represented in Figure 5.1. There were two notable differences between the eighth- and sixth grade models. First, peer support was only a resource for eighth-grade motivation and not sixth-grade motivation. This corresponds to correlational findings, in that between peer support and engagement for eighth graders and the lack of that correlation for sixth graders. Second, eighth graders showed significant gender effects in the levels of the DVs whereas sixth graders did not.

\section{Research question 2c: significantly more salient for eighth graders than}

for sixth graders? In order to formally test whether the sixth graders differed from the eighth graders in their relationships between the developmental resources and motivational resilience group, a 3 × 2 MANCOVA was used to assess whether mean levels of the four developmental resources differed as a function of resilience group and grade in the combined sample of eighth graders and sixth graders. The independent variables were motivational resilience group (resilient vs. average vs. 
stress-affected), grade (sixth vs. eighth), and a resilience group by grade interaction term. Gender (male vs. female) was included as a covariate. Of most interest was the possible interaction between the two IVs, motivation resilience group (resilient vs. average vs. stress-affected) and grade level (sixth vs. eighth), investigating whether the patterns among motivational resilience groups differed at the two grade levels. Also of interest was the effect of grade, investigating whether the mean differences in developmental resources differed by grade. Mean levels and post-hoc results can be found in Appendix B.

The overall MANCOVA showed a significant main effect for motivational resilience group, Pillai's Trace $=.39, F(8,626)=19.11, p<.001$, partial $\eta^{2}=.20$, after controlling for the significant effect of gender, Pillai's Trace $=.06, F(4,312)=5.48, p$ $<.001$, partial $\eta^{2}=.07$. Univariate follow-up ANOVAs showed a similar pattern of effects in this combined-sample model as with the eighth-grade model, with all four developmental resources appearing to serve as motivational resources. There was also a significant effect for grade level, Pillai's Trace $=.09, F(4,312)=7.36, p<.001$, partial $\eta^{2}=.09$. Specifically, univariate follow-up ANOVAs showed that eighth graders had lower levels of garden engagement than eighth graders. The grade-by-group interaction term was not significant, Pillai's Trace $=.02, F(8,604)=.69, p<.05$, partial $\eta^{2}=.01$, meaning that there was no evidence that the differences in the levels of the developmental resources among the motivational resilience groups were greater than those for sixth graders.

Summary of research question 2. Research question two showed that 
autonomy, teacher support, peer support, and garden engagement all appeared to serve as resources for eighth graders motivational resilience, with autonomy and teacher support as the strongest resources. The sixth grade model appeared to differ from the eighth-grade model in that peer support was not a resource (and that there was no effect of gender on the DVs for sixth graders). The combined model, however, did not show that the relationship between the developmental resources and motivational resilience group differed significantly between grades.

\section{Research Question 3: Academic Resilience Groups}

Do levels of the four developmental resources (autonomy, teacher support, peer support, and garden engagement) differ as a function of academic resilience group?

Whereas research question two examined mean differences in levels of four developmental resources as a function of motivational resilience group (based on student engagement), research question three investigated differences as a function of academic resilience group (based on student GPA.) As with in question two, a series of three MANOVAs investigated these relationships in eighth graders, sixth graders, and in the combined sample of both age groups to investigate whether the pattern of differences varied between grades. Mean levels of the four developmental resources at each level of academic resilience group are presented in Appendix B, and significant mean-level differences for both grades are represented in Figure 5.2.

Research question 3a: for eighth graders? A one-way MANCOVA was used to test whether levels of the four developmental resources (autonomy, teacher support, peer support, and garden engagement; the dependent variables) differed as a function 
Figure 5.2. Mean Levels of Autonomy, Teacher Support, Peer Support, and Garden Engagement in Eighth-grade Academic Resilience Groups.

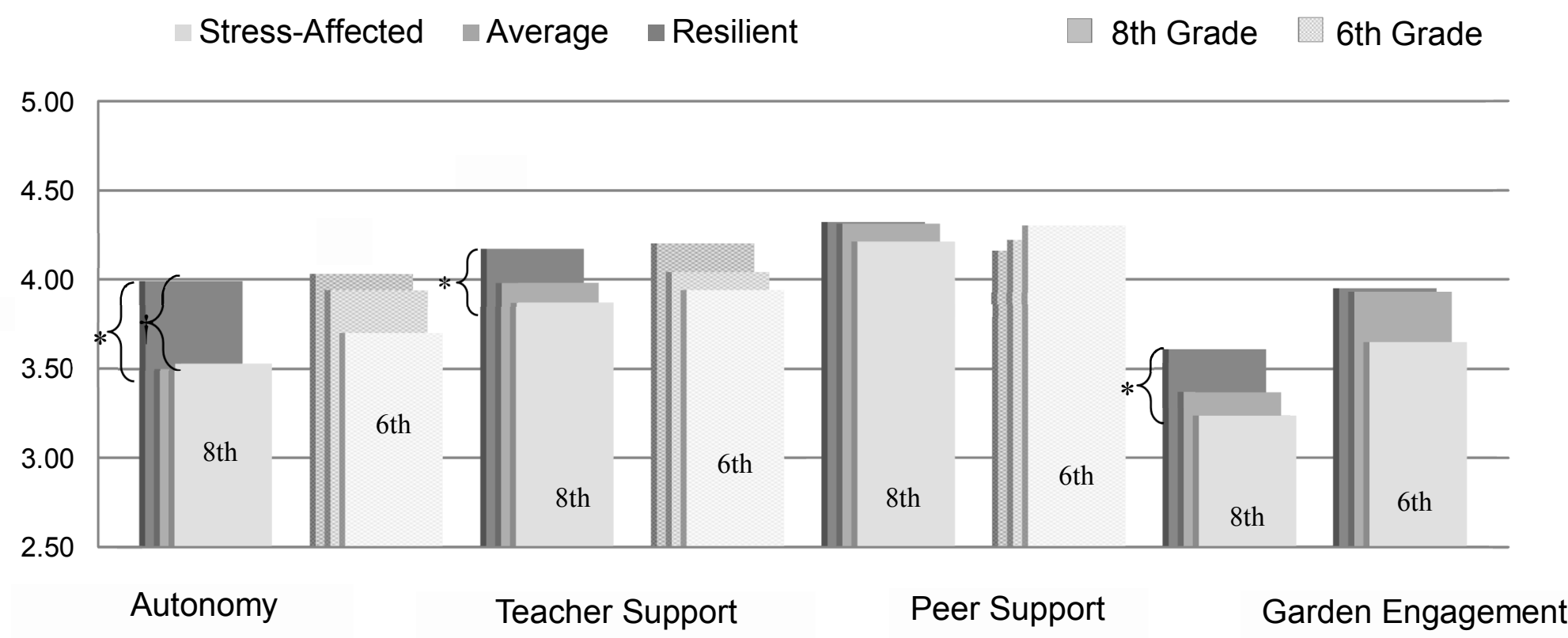

Note: $n=167$. Bars show mean levels of autonomy, teacher support, peer support, and garden engagement in eighthgrade academic resilience groups. Mean levels of each developmental resource are clumped, with resilient groups in rear and stress-affected groups in front. Significant differences between groups shown by bracket and $*$ for $p<.05$ and $\dagger$ for $p<.10$. 
of academic resilience group. The independent variable was academic resilience group (stress-affected vs. average vs. resilient). Gender (male vs. female) was entered as a covariate. There was significant effect of academic resilience group on the combined DVs, Pillai's Trace $=.10, F(8,318)=1.99, p=.05$, partial $\eta^{2}=.05$, after controlling for the significant effect of gender on the combined DVs, Pillai's Trace $=.05, F(4,158)=$ 3.63, $p<.01$, partial $\eta^{2}=.08$. Follow-up ANCOVAs showed that levels of autonomy, teacher support, and garden engagement all differed between academic resilience groups, after controlling for gender. Post-hoc tests showed that autonomy, teacher support, and garden engagement all served as general academic resources, with higher levels in the resilient group than in the stress-affected group. Only autonomy appeared to serve as a (marginally significant) enabling factor, with higher levels of autonomy in the resilient group than in the average group. No resources appeared to serve as protective resources. There were no significant group differences in the levels of peer support, which corresponded to the finding that peer support was not correlated with GPA.

\section{Research Question 3b: more salient for eighth graders than for sixth}

graders? A one-way MANOVA was used to assess mean level differences in the four developmental resources (the dependent variables) within sixth graders, for the purposes of comparing these relationships with those of the eighth graders. The independent variable was academic resilience group (stress-affected vs. average vs. resilient). The omnibus test did not show a significant effect for academic resilience group on the levels of combined DV, Pillai's Trace $=.09, F(8,294)=1.67$, n.s., partial 
$\eta^{2}=.04$. It was unexpected to find no significant effect for academic resilience group at the multivariate level.

\section{Research question 3c: significantly more salient for eighth graders than}

for sixth graders? A 3 x 2 MANCOVA was used to assess mean level differences in the four developmental resources (the dependent variables) as a function of academic resilience group in the entire sample of eighth- and sixth-graders. The independent variables were academic resilience group (resilient vs. average vs. stress-affected) followed by grade (sixth vs. eighth), and a resilience group by grade interaction term. Gender (male vs. female) was included as a covariate. Of most interest was the possible interaction between the two IVs, academic resilience group (resilient vs. average vs. stress-affected) and grade level (sixth vs. eighth), investigating whether the patterns among academic resilience groups differed at the two grade levels. Also of interest was the effect of grade, investigating whether the mean differences in developmental resources were lower for eighth graders than for sixth graders. The overall MANCOVA showed a significant main effect for academic resilience group, Pillai's Trace $=.10, F(8,616)=3.83, p<.001$, partial $\eta^{2}=.05$, after controlling for the significant effect of gender, Pillai's Trace $=.05, F(4,307)=3.98, p<.01$, partial $\eta^{2}$ $=.05$. Follow-up ANCOVAs found significant effects for academic resilience group on levels of autonomy, teacher support, and garden engagement, after controlling for gender.

There was also a significant effect for grade level, Pillai's Trace $=.09$, $F(4,307)=7.96, p<.001$, partial $\eta^{2}=.09$. Follow-up ANCOVAs found significant 
grade effects for autonomy and garden engagement, such eighth-grade students' levels were lower than sixth-grade students', after controlling for the effect of gender. The grade by group interaction term was not significant, Pillai's Trace $=.02, F(8,616)=$ .95 , n.s., partial $\eta^{2}=.01$. Thus, the major hypothesis of this research question was not supported.

Summary of research question 3. Autonomy, teacher support, and garden engagement all emerged as resources for eighth-grade academic resilience. Whereas, for motivational resilience, autonomy and teacher support appeared to be stronger resources than the other developmental resources, no resource appeared to be a particularly strong academic resource. There appeared to be some evidence that the developmental resources might be more important academic resources for eighth graders than for sixth graders in that there was no significant multivariate academic resilience group effect for the sixth graders. When tested formally in the combinedgroups model, however, the grade-by-group interaction term was not significant, showing that the resources were not significantly stronger resources for the eighth graders.

\section{Research Question 4: Dual Resilience Groups}

Do levels of the four developmental resources (autonomy, teacher support, peer support, and garden engagement) differ as a function of dual resilience group?

This research question investigated mean differences in levels of four developmental resources as predicted by dual resilience group membership. Whereas research question two investigated motivational resilience, based on student 
engagement, and research question investigated academic resilience, based on student GPA, this question investigated the intersection of motivational and academic resilience, with students who belong to both the motivationally and academically resilient groups forming the dual resilience group, and those who belong to both the motivationally and academically stress-affected groups forming the dually stressaffected group. Mean levels and post-hoc test results can be found in Appendix B. Significant mean-level differences are represented in Figure 5.3.

Research question 4a: for eighth graders? A 2 x 3 factorial MANOVA was used to assess mean level differences in four developmental resources (autonomy teacher support, peer support, and garden engagement; the dependent variables) as a function of dual resilience group in eighth-grade students. The independent variables were gender (male vs. female, entered first in the model) and dual resilience group (stress-affected vs. average vs. resilient). There was a significant effect for dual resilience group, Pillai's Trace $=.20, F(8,318)=4.39, p<.001$, partial $\eta^{2}=.10$. Autonomy, teacher support, and garden engagement all appeared to serve as general resources, in that mean levels were higher in the dually resilient group than in the dually stress-affected group. Additionally, all three resources appeared to serve an enabling role, in that levels of autonomy and teacher support were marginally higher, and levels of garden engagement were significantly higher, in the dually resilient group than in the average group. Autonomy and teacher support also appeared to serve as protective resources, in that levels of these two resources were significantly higher in the average group than in the stress-affected group. There was also a significant 
Figure 5.3. Mean Levels of Autonomy, Teacher Support, Peer Support, and Garden Engagement in Eighth-and Sixth-grade Dual Resilience Groups.

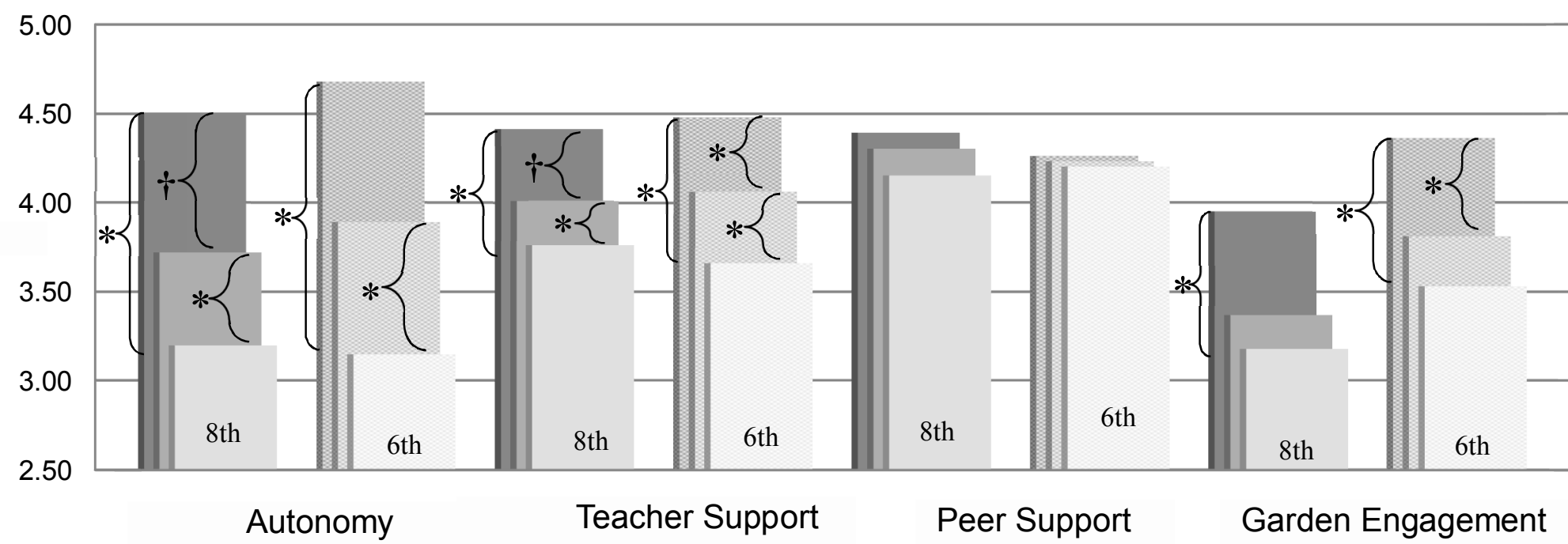

Note: Total $n=322$. Eighth-grade $n=167$, sixth-grade $\mathrm{n}=155$. Bars show mean levels of autonomy, teacher support, peer support, and garden engagement in eighth- and sixth-grade dual resilience groups. Mean levels of each

developmental resource for each grade are clumped, with resilient groups in rear and stress-affected groups in front. Significant differences between groups shown by bracket with $*$ for $p<.05$ or $\uparrow$ for $p<.10$. 
main effect for gender (the relationship between gender and the levels of the four developmental resources can be found RQ2a.)

\section{Research question 4b: more salient for eighth graders than for sixth}

graders? A one-way MANOVA was used to assess mean level differences in the four motivational resources within sixth graders, for the purposes of comparing these relationships with those of the eighth graders. The IV was dual resilience group (stressaffected vs. average vs. resilient). The omnibus test showed that dual resilience group membership had a significant effect on the levels of combined DVs, Pillai's Trace $=.24$, $F(8,300)=5.20, p<.001$, partial $\eta 2=.12$. The pattern of mean-level differences in sixth graders was generally similar to that for eighth graders, thus not providing support for the hypothesis that the developmental resources were more salient for eighth graders.

\section{Research question 4c: eighth grade pattern significantly more pronounced?}

A $2 \times 3 \times 2$ MANOVA conducted in the combined sample investigated whether the eighth-grade pattern of mean-level differences in the four developmental resources (the dependent variables) among dual resilience groups varied between eighth- and sixthgrade students. The independent variables were gender (male vs. female), entered first in the model, followed by dual resilience group (resilient vs. average vs. stressaffected), and then by grade (sixth vs. eighth), and, finally, a grade by resilience group interaction term. Significant effects for dual resilience group confirmed those found in the sixth- and eighth-grade models, and significant effects for grade and gender confirmed those found in the motivational and academic resilience group tests. The grade-by-group interaction term was not significant, again demonstrating that there was 
no evidence that the pattern of dual resilience group differences for eighth graders was stronger for eighth graders than for sixth graders.

Summary of research question 4. Overall, the pattern of results for dual resilience is similar to those for motivational and academic resilience. The developmental resources seemed to differ more as a function of dual resilience group than as a function of academic resilience group alone, and dual resilience groups differed from motivational resilience groups mainly in that levels of peer support did not differ as a function of dual resilience group. As expected, eighth-grade students showed lower levels of the developmental resources than did sixth-grade students (specifically, of autonomy and garden engagement), but the grade by resilience group interaction term was not significant for any of the three types of resilience examined here, indicating no formal difference between grades in the relationship between resilience group and developmental resources.

At the same time, the relationships between the developmental resources and the various resilience groups did differ somewhat between eighth- and sixth-grade students. Specifically, a) peer support was a motivational resource for eighth graders and not sixth graders; b) the development resources differed as a function of eighth-grade academic resilience group, but not sixth-grade academic resilience group students; and c) the developmental resources differed significantly as a function of gender for eighth graders and not for sixth graders. 


\section{Research Questions 5: Enabling and Protective Factors Predicting Dual Resilience Group Membership}

Do each of the developmental resources predict dual resilience group membership?

To answer this question, the final set of analyses used a series of logistic regressions to examine whether the four developmental resources (autonomy, teacher support, peer support, and garden engagement) predicted dual resilience group membership, and whether the pattern of prediction varied by grade. We investigated these relationships in eighth-grade and compared those relationships to those for sixthgrade students. This research question had two subsections: first, testing the unique effects for each developmental resource in predicting dual resilience group, when controlling for the other resources; and second, testing whether autonomy mediated the relationships between the remaining three developmental resources (teacher support, peer support, and garden engagement) and dual resilience group membership.

This question focused on the enabling and protective roles of each resource in regard to dual resilience. We asked whether each resource predicted students being more likely to be member of the dually resilient group than the average group (suggesting an enabling role), and whether each resource predicts being less likely to be a member of the dually stress-affected group than the average group (suggesting a protective role.)

All analyses were conducted in Mplus using a montecarlo integration procedure. Missing data were estimated using a maximum likelihood method with robust standard errors (MLR.) To control for gender, a gender term was included as an additional 
independent variable in all models. Each of the developmental resources were grandmean centered to address multicollinearity between the resources.

Research Question 5a: Do each of the four developmental resources, with special focus on autonomy and garden engagement, uniquely predict dual resilience group membership for when controlling for all other developmental resources?

In this research question, a multi-group multinomial logistic regression model was created in which the four developmental resources and gender were independent variables. The dependent variable was dual resilience group membership, which was treated as a nominal variable with two predicted outcomes: dually resilient (vs. average) or dually stress-affected (vs. average) group membership. Grade was used as the grouping variable. In the first part of this question, we examined a model in which parameters were allowed to vary between these grade groups in order to compare estimates for eighth graders with estimates for sixth graders. Collectively, the four developmental resources and gender provided significant utility in predicting dual resilience group membership when compared to an intercepts-only model, $X^{2}(20)=$ $94.05, p<.001$.

Although we were interested in each of the developmental resources, we were especially interested in whether autonomy and garden engagement might emerge as stronger unique predictors for eighth-grade students than for sixth-grade students. We thought that for the eighth graders, autonomy (which measures students' identified reasons for doing schoolwork) might serve as a driver for the other inter-personal resources for eighth graders. We also thought that with authentic and purposeful work 
outside the normal classroom setting might give students an alternative pathway through which to experience positive motivational processes, even if the rest of their school experiences did not inspire commitment.

Research question 5a1: unique effects for eighth graders? For eighth-grade students, autonomy emerged as the only significant unique predictor of dual resilience group $^{1}$. Correlations between the IVs and odds ratios predicting dual resilience group are shown in Figure 5.4. Regression slopes, $p$-values, standard errors, $z$-scores, odds ratios, and confidence intervals are reported along with model fit information in Appendix B.

As hypothesized, autonomy uniquely predicted resilience group membership for

${ }^{1}$ In this and the other regressions, there were marginally significant or significant effects for peer support in the opposite direction expected, implying suppression effects. For this analysis, there was a marginally significant $(p=.10)$ effect for peer support, such that an increase in peer support corresponded to a decreased likelihood of being in the resilient group, after controlling for gender and the other developmental resources. Because peer support was correlated positively with engagement for eighth graders, was not significantly correlated with either engagement or achievement for sixth graders, and was positively associated with other developmental resources in this model, it was difficult to interpret this contradictory effect. We therefore interpreted this recurrent finding to be inconclusive regarding peer support as either an enabling or protective factor for sixth graders. 
Figure 5.4. Mutli-group Multinomial Logistic SEM: Grade Differences in Unique Effects of Developmental Resources on Dual Resilience Group Membership.
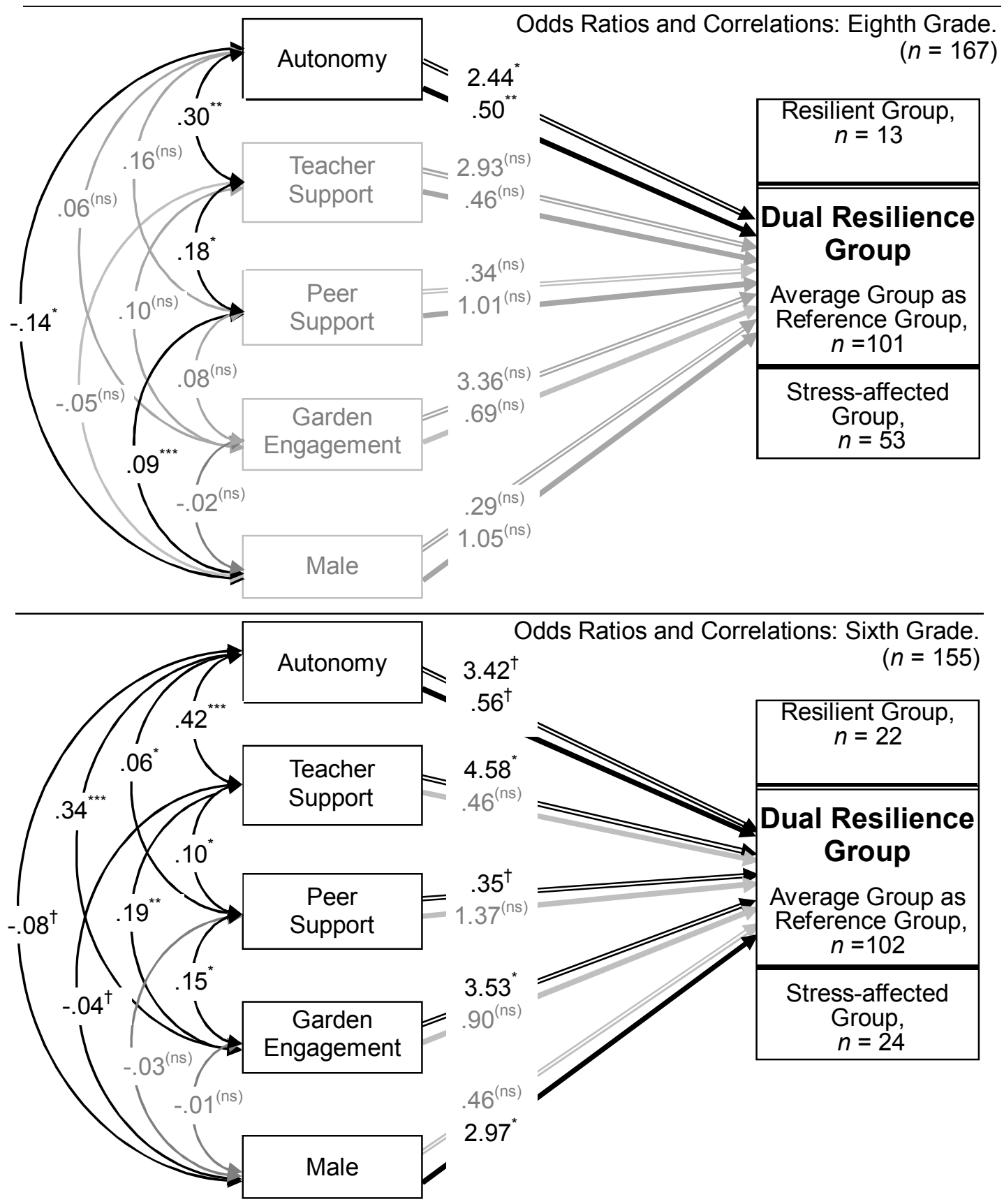

Note: $n=322 . \quad{ }^{n * \pi *} p<.001,{ }^{* *} p<.01,{ }^{*} p<.05,{ }^{\dagger} p<.10,{ }^{(\mathrm{ns})} p<.10$. Grey coloring indicates nonsignificant path or correlation. Hollow $(=$ ) lines indicate paths predicting dually resilient (vs. average) group membership; solid $($ ) lines indicate paths predicting dually stress-affected (vs. average) group membership. Values on paths are the odds of being in the selected group (vs. the average group) that correspond to each unit increase in the IV, where $>1$ is increased likelihood, 1 is equal likelihood, and $<1$ is decreased likelihood. Gender coded such that female $=0$ and male $=1$. All developmental resources grand mean centered. 
eighth-graders, after controlling for teacher support, peer support, garden engagement, and gender. A one-unit increase in levels of autonomy was marginally associated with it being 2.44 times as likely for students to be members of the resilient group as it was to for them to be members of the average group, $p=.05$. Each unit increase in autonomy was also associated with it being .50 times (one half) as likely for students to be members of the stress-affected group as it was for them to be members of the average group, $p=.003$.

The remaining developmental resources, however, did not uniquely predict group resilient or stress-affected group membership after controlling for the other variables, nor did gender (see Appendix c.) Thus, hypothesis 5a1 was partially supported, in that autonomy both appeared to enable resilient (vs. average) group membership, and to protect against stress affected (vs. average) group membership, even after controlling for all other variables. Because no other developmental resources were significant predictors of dually resilient or stress-affected group membership after controlling for autonomy (and the other variables,) these findings lend support to the idea that autonomy might serve as a driving resource for eighth-grade students. Contrary to our hypothesis, however, garden engagement did not uniquely predict eighth-grade dually resilient or stress-affected group membership after controlling for the other variables.

\section{Research question 5a2: stronger unique effects for eighth graders than for}

sixth graders? In the sixth-grade portion of the model, three of the four developmental resources uniquely predicted resilience group membership. Correlations between the 
independent variables and odds ratios predicting dual resilience group are shown in Figure 5.4. Logistic regression coefficients, $p$-values, standard errors, and confidence intervals are shown along with odds ratios in Appendix C.

For sixth graders, autonomy was a marginally significant predictor of resilience group membership. Teacher support was the strongest unique predictor of resilience group membership for sixth graders, after controlling for the other variables. This contrasts the results for eighth graders, for whom teacher support did not uniquely predict resilient or stress-affected group membership. Garden engagement was also a significant predictor of dually resilient group membership for sixth graders, after controlling for the other variables. Finally, gender was a significant unique predictor of dual resilience group for sixth graders (such that being male was associated with it being 2.97 times as likely for students to be members of the stress-affected group as it was for them to be members of the average group).

Thus, the only small way in which hypothesis $5 \mathrm{a} 2$ supported in that autonomy was a significant predictor of dual resilience group for eighth graders and only a marginally significant predictor for sixth graders. Otherwise, the developmental resources appeared to be, if anything, stronger predictors of dual resilience for sixth graders, and thus hypothesis $5 \mathrm{~b} 2$ was generally not supported. One unexpected difference between grades was that, despite eighth graders having generally larger mean differences between male and female students than sixth graders, gender was only a unique predictor of dual resilience group for sixth graders. This could suggest that the relationship between gender and dual resilience group might have been mediated by one 
of the developmental resources for eighth graders, but not for sixth graders.

Research question 5a3: Do the four developmental resources differ significantly as unique predictors of dual resilience group membership for eighth-grade students in comparison to sixth-grade students? Invariance testing was used to compare the sixth- and eighth- grade models in regard to their prediction of dual resilience group membership. There are traditionally three steps for model invariance testing in multigroup models (Tabachnik \& Fidel, 2007). In the first step, a configural model is analyzed, leaving all parameters unconstrained between the groups. The second step of this process applies constraints to the measurement model, with the intention of establishing measurement invariance or partial measurement invariance. If invariance is supported, the third and final step tests the utility of allowing specific model parameters to vary between groups. Because this was only a structural model, the full model invariance testing process could not be observed. The modified steps taken are described below.

The first step, creating an unconstrained model in which parameters were allowed to vary between the sixth- and eighth-grade groups, was described in RQ5a1 and RQ5a2. Because this model had no explicit measurement component, invariance of factor loadings between groups could not be assessed. In order to investigate the extent to which there might be invariance between other variable properties, we constrained variances for the IVs to be equal between sixth- and eighth-grade groups. No significant reductions in fit were revealed when compared with the unconstrained model, $X^{2}(5)=$ $3.15, p=.66$, which suggests that, regarding the variances of the IVs, invariance could 
be assumed between the sixth- and eighth-grade groups.

Next, we tested invariance in the correlations between the two grades.

Constraining the correlations among the developmental resources and gender to be equal across the grade groups did result in a significant reduction in model fit when compared with the unconstrained model, $X^{2}(15)=28.95 p=.02$. Several correlations (teacher support with peer support, teacher support with garden engagement, and peer support with gender) seemed to cause a small reduction in model fit when compared with a model where only variances were constrained to be equal between the groups. Two correlations caused significant reductions in model fit when compared with the constrained variances model: autonomy with teacher support and autonomy, with a coefficient of $r=.27$ for eighth graders and $r=.47$ for sixth graders, and autonomy with garden engagement, which was not significant for eighth graders and $r=.34$ for sixth graders. Of these, the correlation between autonomy and teacher support caused a marginally significant reduction in model fit when compared with the unconstrained model. When the constraint for this correlation was released, allowing it to vary across grade groups, the resulting model did not have significantly worse fit in comparison to the unconstrained model, $X^{2}(14)=20.61, p=.11$. Thus, we could assume partial structural invariance for the correlations among the IVs between the sixth- and eighthgrade groups.

Finally, we tested our hypothesis that eighth graders would differ significantly from sixth graders in terms of the regression paths between the developmental variables and dual resilience group. Constraining the regression paths from the four 
developmental resources (and gender) to dual resilience group to be equal across grade groups, however, did not significantly reduce model fit, $X^{2}(24)=25.25, p=.39$. In

finding that there did seem to be structural invariance for regression paths across grade groups, our hypothesis that the developmental resources differed as predictors of dual resilience group for the two grades was not supported.

Research Questions 5b: Does autonomy mediate the relationships between the three remaining developmental resources (teacher support, peer support, and garden engagement) and dual resilience group membership?

In order to directly investigate the idea that autonomy might serve a central role in dual resilience for eighth graders, becoming a driver of the other resources, we tested a series of mediated logistic regressions. A set of preliminary analyses tested separate regression models for each developmental resource as a predictor of dual resilience group in order to identify candidates for mediation analyses. The results of the preliminary analyses can be found in Appendix D. In order to facilitate the interpretation of the mediation results, separate models were run for comparing dually resilient vs. average and for comparing dually stress-affected vs. average. Gender was included in the models when gender was at least a marginally significant predictor of dual resilience group in the preliminary analyses. We conducted these analyses first for eighth graders, and then compared those results to those for sixth graders.

These analyses took the following sequence for each comparison, based on both Baron \& Kenny's (1986) causal steps method and Mackinnon \& Dwyer's (1993) modification of the product of coefficients method for calculating the indirect effect. We 
first followed Baron \& Kenny's four-step method: First, the preliminary analyses showed whether each developmental resource predicted dual resilience group membership. We proceeded to test mediation in the models in which developmental resources were significant marginally predictors of dual resilience group. At this point, we redefined those models so that the dependent variable was dichotomous, either resilient vs. average or stress-affected vs. average. In the second step, we tested whether the developmental resource was a significant predictor of autonomy. In the third step, we tested whether autonomy was still a significant predictor of dual resilience group when controlling for the developmental resource and gender. In the fourth step, we tested whether the relationship between the developmental resource and dual resilience group was reduced (which would imply partial mediation according to Baron and Kenny's 1986 guidelines) or eliminated (which would imply full mediation) when controlling for autonomy. Finally, we tested the mediated relationship with Sobel's (1982) test of indirect effects. We used a method for standardizing coefficients and calculating indirect effects that was modified for analyses categorical outcomes (Mackinnon \& Dwyer, 1993; Herr, 1996)

Research question 5b1: for eighth graders? Preliminary analyses were used to identify which developmental resources could be tested for mediation. These analyses showed that teacher support predicted both dually resilient and stress-affected group membership, and that garden engagement predicted dually resilient group membership. Mediation analyses continued for these developmental resources.

Eighth-grade teacher support: resilient group. In the preliminary analyses, we 
saw that both teacher support and gender marginally predicted the likelihood of being in the resilient group rather than the average group, so both variables were included in the mediation analyses. Model fit information and standardized coefficients are reported in Figure 5.5. In the first step, we redefined this model to predict the dichotomized outcome variable of dually resilient vs. average group, and confirmed that teacher support was associated with dually resilient group, $(\beta=.36, p=.06)$, and found that gender was marginally significantly associated with dually resilient group, $(\beta=-.30, p$ $=.10$ ). In the second step of the analysis, we found that teacher support was positively associated with autonomy $(\beta=.22, p=<001)$, and that gender was not associated with autonomy $(\beta=-.09, p=.12)$. In the third step, autonomy was positively associated with dually resilient group membership after controlling for teacher support, $(\beta=.35, p=$ .06). In the fourth step, teacher support was no longer significantly associated with dually resilient group membership $(\beta=.26, p=.16)$, after controlling for autonomy and gender $(\beta=-.28, p=.13)$. The indirect effect of teacher support to dual resilience group through autonomy was marginally significant, $(\beta=.08, p=.09)$. Thus, there is 
Chapter 5: Results

Figure 5.5. Mediated Logistic Regression: Autonomy as a Mediator between Eighth-Grade Dual Teacher Support and Dual Resilience Group.

Eighth-grade: Standardized coefficients.

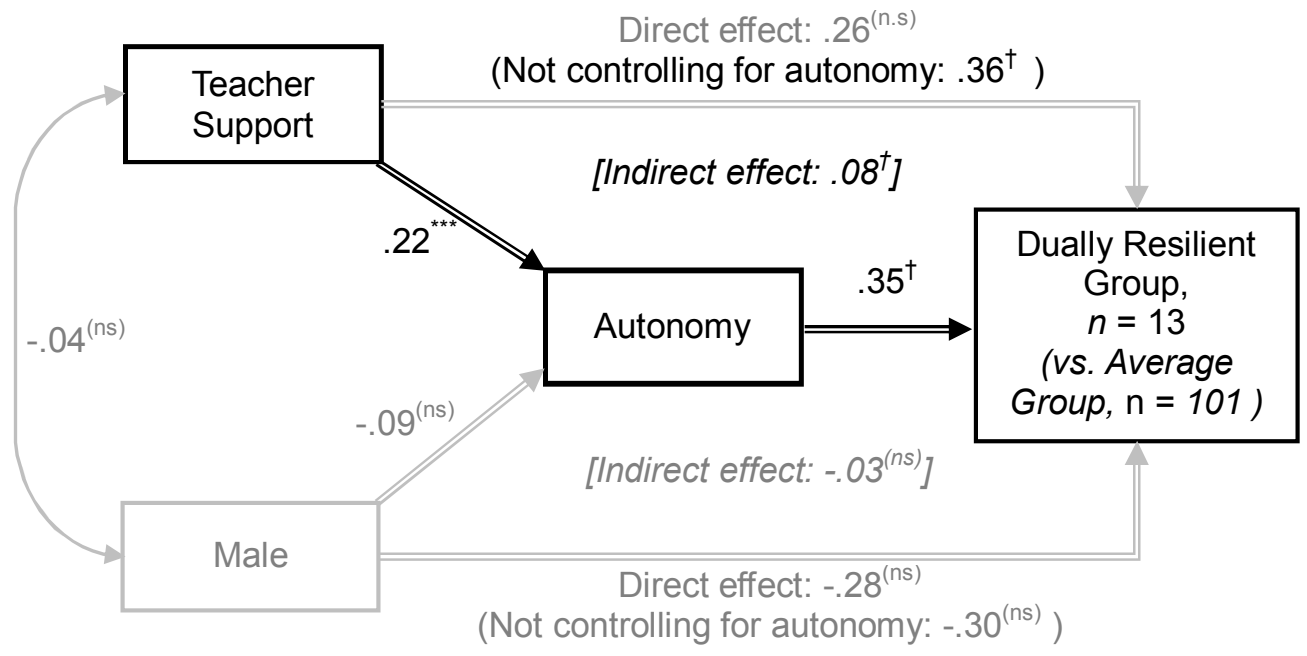

Model information:

1:Teacher Support $->$ Dually Resilient: $X^{2}(2)=8.02, p=.02$.; $A I C=388.14$, BIC $=410.03$.

2: Teacher Support $->$ Autonomy: $X^{2}(2)=12.86, p=.002 ;$ AIC $=527.41$, BIC $=552.04$.

3:Teacher Support \& Autonomy $->$ Dually Resilient: $X^{2}(3)=12.02, p=.007 ;$ AIC $=605.39$, $B I C=640.96$.

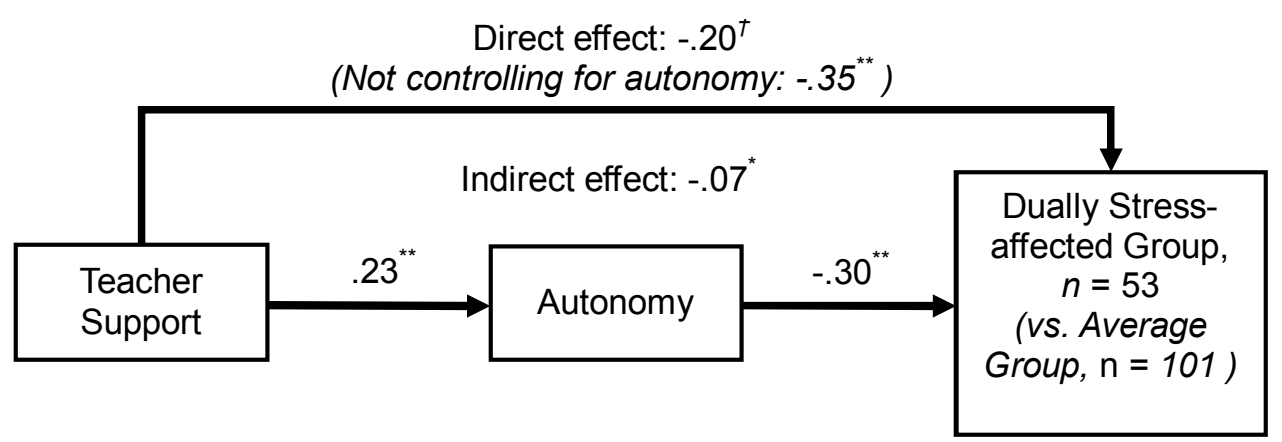

Model information:

1:Teacher Support $->$ Dually Stress-affected: $X^{2}(1)=8.56, p=.03 ; A I C=382.85, B I C=395.00$.

2: Teacher Support $->$ Autonomy: $X^{2}(1)=6.91, p=.009 ; A I C=468.66, B I C=481.16$.

3:Teacher Support \& Autonomy - > Dually Stress-affected: $X^{2}(2)=20.79, p<.001 ;$ AIC $=654.10$

, $B I C=678.40$

Note: Total $n=155 ; n$ for stress-affected $v$ s. average $=134, n$ for resilient $v$ s. average $=154$.

${ }^{* * *} p<.001,{ }^{* *} p<.01,{ }^{*} p<.05,{ }^{\dagger} p<.10,{ }^{\text {(ns) }} p<.10$. Parentheses indicate paths from IVs to dual resilience group when not controlling for autonomy. Grey coloring indicates non-significant paths and correlations. Gender was omitted from the model. Teacher support was grand-mean centered. 
evidence that the relationship between teacher support and dually resilient group membership was fully mediated by autonomy for eighth graders, using Barron \& Kenny’s (1986) criteria.

Eighth-grade teacher support, stress-affected group. We followed the same series of steps to investigate the mediation of the relationship between teacher support and stress-affected group status. (For this and all subsequent mediation analyses, a detailed description of each step with beta weights can be found in Appendix D.) In the first step, we found that teacher support significantly predicted dually stress-affected group membership. Teacher support also significantly predicted levels of autonomy, and when autonomy was added to the model, the effect of teacher support on dually stressaffected group membership decreased to being only marginally significant, but was still present. The indirect effect of teacher support on dually stress-affected group membership through autonomy was significant. Thus, it appears that autonomy partially mediated teacher support in its protective role against stress-affectedness.

Eighth-grade garden engagement, resilient group. In the preliminary analyses, we saw that both garden engagement and gender marginally predicted the likelihood of being in the resilient group instead of the average group, so both variables were included in the mediation analyses. Garden engagement did not predict autonomy (which is unsurprising, given that garden engagement and autonomy were not correlated for eighth graders.) Thus, autonomy did not mediated garden engagement in its role 
Figure 5.6. Mediated Logistic Regression: Autonomy as a Mediator between Eighth-Grade Garden Engagement and Dually Resilient Group.

\section{Eighth-grade garden engagement: standardized coefficients.}

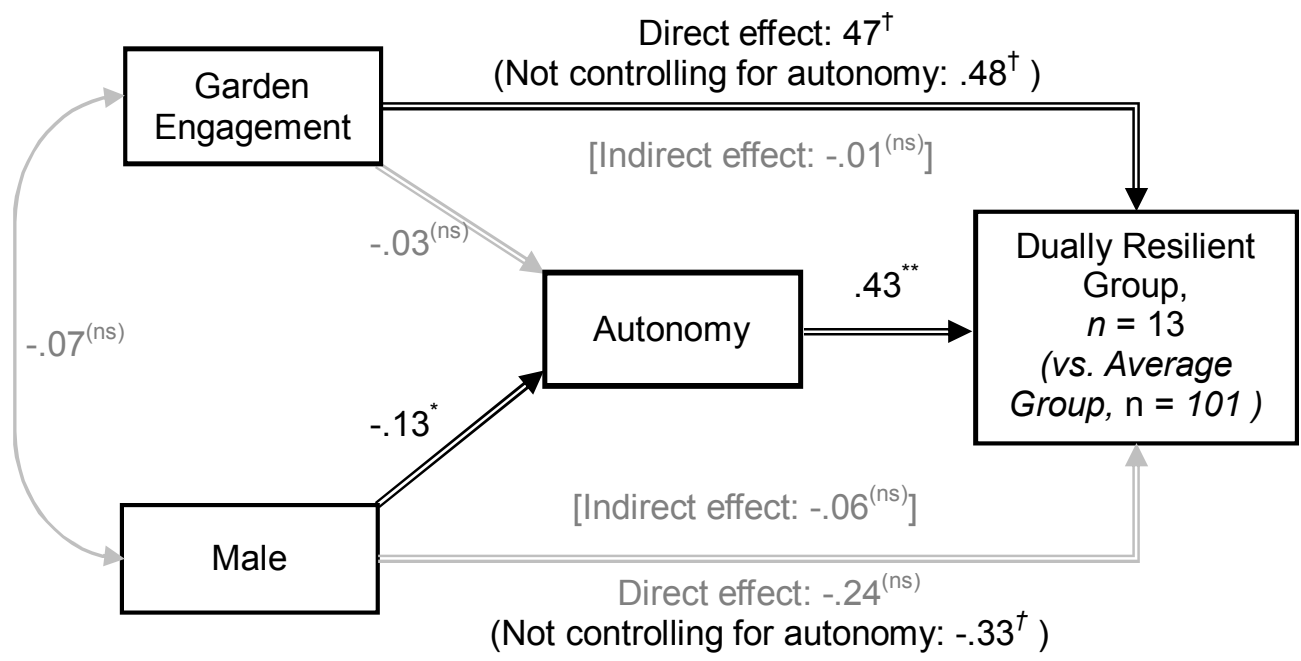

Model information:

1:Garden Engagement $->$ Dually Resilient: $\left(X^{2}(2)=7.23, p=.03 ; \mathrm{AIC}=474.69, \mathrm{BIC}=496.694\right.$.

2: Garden Engagement -> Autonomy: $X^{2}(2)=3.55, p=.17 ; A I C=630.02, B I C=654.64$.

3:Garden Engagement \& Autonomy -> Dually Resilient: $X^{2}(3)=13.27, p=.004 ; A I C=702.33$, $B I C=737.904$.

Note: $n=125 .{ }^{* * *} p<.001,{ }^{* *} p<.01,{ }^{*} p<.05,{ }^{\dagger} p<.10,{ }^{(\mathrm{ns})} p<.10$. Grey coloring indicates nonsignificant paths and correlations. Gender was coded such that female $=0$ and male $=1$. Garden engagement was grand-mean centered. 
enabling resilient group membership. Unexpectedly, in the same model, autonomy did appear to mediate the relationship between gender and resilient group membership.

\section{Research question 5b2: autonomy a stronger mediator for eighth graders}

than for sixth graders? The same set of preliminary analyses and meditational analyses was repeated for sixth graders to determine whether autonomy appeared to be more of a mediator for eighth graders than for sixth graders. In the preliminary analyses, teacher support significantly predicted both resilient and stress-affected group membership, and garden engagement significantly predicted resilient group membership and marginally predicted stress-affected group membership. The meditational analyses found that autonomy partially mediated teacher support both in its role as an enabling resource for resilient group membership and in its role as a protective resource against stress affectedness. (In comparison, for eighth graders, autonomy fully mediated teacher support as an enabling resource and partially mediated it as a protective resource.) Autonomy also partially mediated garden engagement in its role as an enabling resource for resilient group membership and fully mediated garden engagement in its role as a protective resource against stress-affectedness. (For eighth graders, autonomy did not mediate garden engagement.) Results of these analyses can be found in Figures 5.7 and 5.8.

Thus, there was only weak partial support for this hypothesis. Autonomy did appear to be a stronger mediator of eighth-grade teacher support as an enabling resource for dual resilience in that, for eighth graders, autonomy fully mediated teacher support 
Figure 5.7. Mediated Logistic Regression: Autonomy as a Mediator between Sixth-Grade Teacher Support and Dual Resilience Group.

Sixth-Grade Teacher Support: Standardized coefficients.
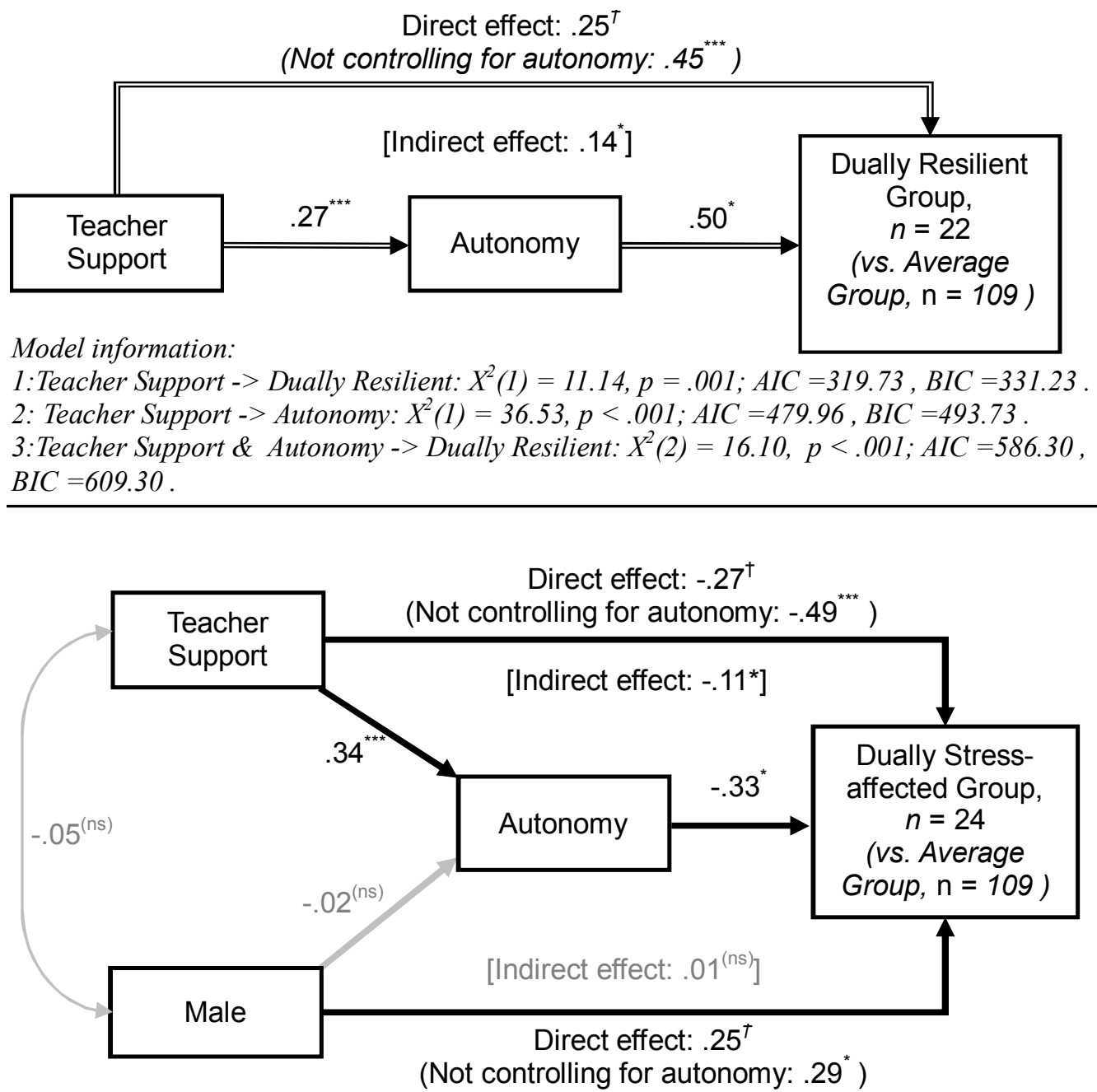

Model information:

1:Teacher Support $->$ Dually stress-affected: $X^{2}(2)=17.86, p<.001 ;$ AIC $=528.41$, BIC $=551.54$.

2: Teacher Support $->$ Autonomy: $X^{2}(2)=56.18, p<.001 ;$ AIC $=687.38$, BIC $=713.39$.

3: Teacher Support \& Autonomy -> Dually stress-affected: $X^{2}(3)=26.18, p<.001$; AIC $=799.24$, $B I C=836.82$.

Note: Total $n=155 ; n$ for stress-affected vs. average $=133, n$ for resilient vs average $=131 .{ }^{* * *} p<$ $.001,{ }^{* *} p<.01,{ }^{*} p<.05,{ }^{\dagger} p<.10,{ }^{\text {(ns) }} p<.10$. Grey coloring indicates non-significant paths and correlations. Gender was coded such that female $=0$ and male $=1$. Teacher support was grand-mean centered. 
Figure 5.8. Mediated Logistic Regression: Autonomy as a Mediator between SixthGrade Garden Engagement and Dual Resilience Group.

\section{Sixth-Grade Garden Engagement: Standardized coefficients}

Direct effect: .27

(Not controlling for autonomy: .43**)

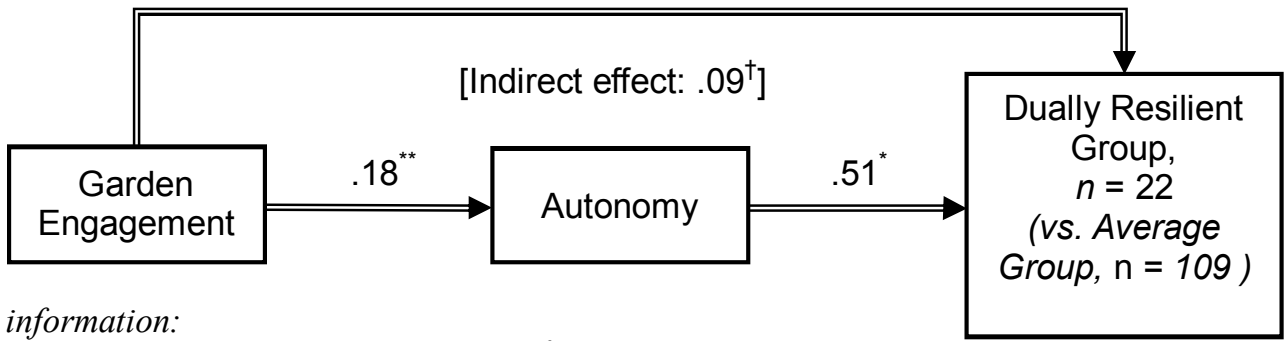

Model information:

1:Garden Engagement $->$ Dually Resilient: $X^{2}(1)=8.69, p=.003 ; A I C=321.75, B I C=333.25$.

2: Garden Engagement $\rightarrow>$ Autonomy: $X^{2}(1)=7.25, p=.007 ; A I C=510.49, B I C=524.55$.

3: Garden Engagement \& Autonomy $->$ Dually Resilient: $X^{2}(2)=20.50, p<.001 ;$ AIC $=617.30$, $B I C=640.30$.

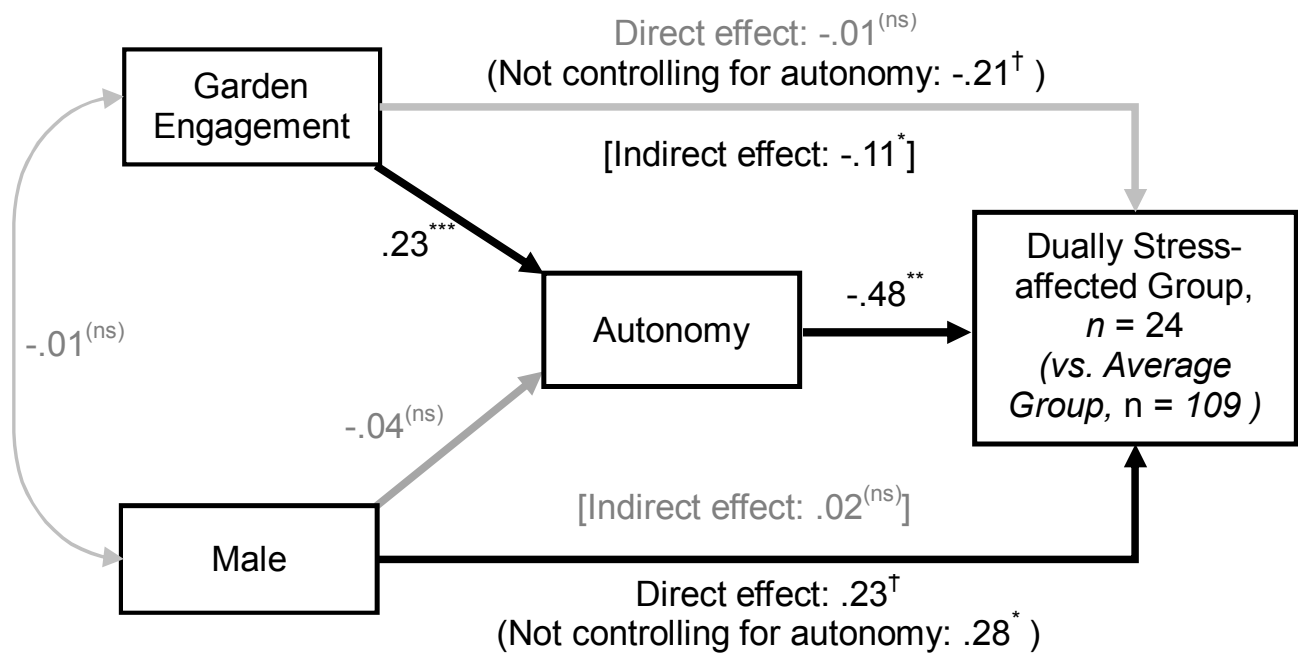

Model information:

1:Garden Engagement $->$ Dually Resilient: $X^{2}(2)=8.00, p=.02 ;$ AIC $=550.89, B I C=574.01$.

2: Garden Engagement $->$ Autonomy: $X^{2}(2)=12.94, p=.002 ; A I C=736.67, B I C=762.68$.

3:Garden Engagement \& Autonomy $->$ Dually Resilient: $X^{2}(3)=19.23, p<.001 ;$ AIC $=852.45$, $B I C=890.02$.

Note: Total $n=155 ; n$ for stress-affected vs. average $=133, n$ for resilient vs average $=131 .{ }^{* * *} p<$ $.001,{ }^{* *} p<.01,{ }^{*} p<.05,{ }^{\dagger} p<.10,{ }^{\text {(ns) }} p<.10$. Grey coloring indicates non-significant paths and correlations. Gender was coded such that female $=0$ and male $=1$. Garden engagement was grandmean centered. 
as an enabling resource, and partially mediated that relationship for sixth graders. In contrast, autonomy had no relationship with garden engagement for eighth graders but fully mediated garden engagement as a protective resource for sixth graders. Thus, counter to expectations, autonomy was more of a mediator of garden engagement for the sixth graders than it was for the eighth graders. 


\section{CHAPTER 6.}

\section{DISCUSSION}

The goal of the current study was to examine the personal and interpersonal predictors of motivational and academic success in young at-risk adolescents, using frameworks supplied by work on stage-environment fit, resilience, and selfdetermination theory. By outlining general processes of resilience, populating those processes with motivationally important factors from SDT and stage-environment fit, and exploring the match of stage and environment by considering the developmental calibration of those processes and factors, this study contributed to an understanding of how demographically at-risk eighth-grade students can be academically and motivationally resilient. By building on the first steps taken in this study, and incorporating new features to address some of the serious limitations this study also faces, future research could make notable steps in understanding and promoting adolescents' motivational resilience. In all, we believe the study serves as a cautious but helpful first step in exploring a developmentally calibrated understanding of resilience in education.

\section{Summary of Findings}

Preliminary analyses of descriptive statistics showed that eighth graders reported moderate or high levels of both the developmental resources (autonomy, teacher support, peer support, and garden engagement) and the markers of resilience (engagement and GPA) with no mean levels falling below the midpoint of the scale. Peer support showed particularly high mean levels; almost all students reported high 
peer support.

The markers of resilience were correlated with almost all of the developmental resources. Engagement showed strong associations with autonomy and teacher support, a moderate association with garden engagement, and a weak relationship with peer support $^{2}$. GPA showed a weak correlation with all developmental resources except peer support, with which there was no significant association. Correlations for sixth graders were similar to, but appeared to be slightly stronger than, correlations for eighth graders, with the exception that sixth graders' peer support was not correlated with engagement.

\section{“Eighth-gradeness" and Developmentally-calibrated Resilience Groups}

The first research question in this study assessed whether "eighth-gradeness" could be considered as an additional motivational and academic risk factor in the cumulative risk context posed by marginalized demographic status and being in a traditional middle school. As expected, eighth-grade students had significantly lower levels of engagement and achievement than sixth-graders in the same context, suggesting that something about "eighth-gradeness" in this context did, indeed, seem to pose an additional risk to students' motivation and achievement (see Table 6.1.)

\footnotetext{
${ }^{2}$ On further investigation, the eighth-grade correlation between engagement and peer support
} was only present for boys, while the eighth-grade correlation between GPA and garden engagement was only present for girls. Additionally, the sixth-grade correlation between GPA and teacher support was also only significant for girls. 
Forming resilience groups. Next, to explore the concept of resilience to the risk of "eighth-gradeness," we used sixth-grade levels of engagement and achievement to inform cutoff levels for eighth-grade motivational and academic resilience groups. Because eighth graders had lower levels of engagement and achievement than sixth graders, the main concern was whether there would be a sufficient number of eighth graders with levels that matched those of resilient-group sixth graders. The smallest group, posing the most concern, was the eighth-grade dually resilient group with only 13 students. However, power analyses indicated that even very small cell sizes might be sufficient to detect the effects investigated in this study. Accordingly, we proceeded cautiously with analyses using developmentally-calibrated resilience groups.

\section{Autonomy, Teacher Support, Peer Support, and Garden Engagement as Motivational and Academic Resources.}

We looked at four developmental resources that we expected to be particularly important for eighth-grade students, and whether these resources seemed to enable motivational and academic resilience or protecting against motivational and academic stress-affectedness. We were also interested in whether all of these relationships with resilience groups were stronger for eighth graders than for sixth graders. To investigate this, we first compared eighth-grade results to sixth-grade results and then tested whether results differed significantly as a function of grade.

ANOVAs: motivational resilience groups. As hypothesized, the analyses of variance conducted on eighth-grade motivational resilience groups showed that differences between resilience groups were present for all four developmental resources, 
and were clearest for autonomy and teacher support. Sixth-grade results were similar, except that peer support did not differ based on resilience group for sixth graders. Thus, there was partial support for the hypotheses that the developmental resources would be more important for eighth graders, if only in regard to peer support. However, in the combined sample of eighth- and sixth-graders, an interaction term testing whether group differences varied as a function of grade was not significant, and thus there was no formal evidence that the developmental resources were more important for eighth graders.

ANOVAs: academic resilience groups. Analyses of variance for academic resilience groups showed less pronounced differences than those for motivational resilience groups. Autonomy, teacher support, and garden engagement all differed as a function of academic resilience groups, but differences were generally only between the most extreme (resilient vs. stress-affected) groups. When compared with sixth graders, these three resources did appear to be more salient for eighth graders in that, collectively, the resources did not differ as a function of sixth-grade academic resilience groups. However, the grade by group interaction term in the combined sample was not significant, and so there was no evidence that the relationship between the resources and academic resilience group differed significantly by grade. 
Table 6.1

Summary of Results.

Note: For RQ 2-5, table indicates whether autonomy, teacher support, peer support, and garden engagement served as enabling resources (if resilient group levels > average group levels), general resources (resilient group > stress-affected group), and/or protective resources (average group > stress-affected group). Parentheses indicate that result was only marginally significant.

\section{Research Question 1: \\ Calibrating resilience. \\ Do mean outcome levels and sample size indicate that use of a developmentally calibrated method for identifying resilience groups would be feasible and, if so, does that method produce adequate numbers of eighth-graders in each resilience group?}

\section{Research Question 2:}

Motivational resilience.

Do levels of the four

developmental resources

(autonomy, teacher

support, peer support, and garden engagement) differ as a function of motivational resilience group?
RQ 1a: "Eighth-graderness." Do eighth graders have significantly lower levels of engagement (motivation) and GPA (achievement) than sixth graders, indicating that "eighth-gradeness" may be a risk factor within this sample of demographically at-risk students in a

traditional middle school?

RQ 1b: Resilience groups. Does the developmentallycalibrated method of forming eighth-grade resilience groups produce eighth-grade groups that are sufficiently large to proceed with analyses?

\section{Yes.}

Yes. The developmentally calibrated eighth-grade groups demonstrated adequate power to detect moderate effects.
RQ 2a: Eighth graders. For eighth-grade students, do the mean levels of the four developmental resources (autonomy, teacher support, peer support, and garden engagement) differ as a function of motivational resilience group?

\section{RQ 2b: Eighth more salient than sixth? Is the pattern} of mean level differences in the four developmental resources as a function of motivational resilience group similar but more pronounced for eighth-grade students than for sixth-grade students?
Yes: All four resources differed based on motivational resilience group for eighth graders.

\begin{tabular}{lllll} 
& Aut. & Tch. Sup. & Peer Sup. & Gard.Eng. \\
\cline { 2 - 4 } & Enabling & Enabling & -- & (Enabling) \\
General & General & General & General \\
& Protective & (Protective) & -- & -- \\
\hline \multirow{2}{*}{ th } & Enabling & Enabling & -- & -- \\
General & General & -- & General \\
& Enabling & Protective & -- & --
\end{tabular}

Partial: Only peer support appeared to be more

important for eighth graders than sixth graders; all other resources showed a similar pattern for both grades. 
RQ 2c: Eighth significantly more salient? Are

differences in mean levels of the developmental assets

between motivational resilience groups greater for eighth

graders than for sixth graders, as shown by a significant
No. The grade*resilience group interaction term was not significant.

grade by motivational resilience group interaction term?

Note: For RQ 2, for eighth graders, the effect of gender appeared to vary by resilience group and thus gender was included as an IV instead of a $C V$, with significant gender effects for autonomy, teacher support (marginally), and peer support. For sixth graders, gender showed no significant effect and was not included as a $C V$.

Research Question 3:

Academic resilience.

Do levels of the four

developmental resources

(autonomy, teacher

support, peer support,

and garden engagement)

differ as a function of

academic resilience

group?
RQ 3a: Eighth graders. For eighth-grade students, do the mean levels of the four developmental resources (autonomy, teacher support, peer support, and garden engagement) differ as a function of academic resilience group?

\section{RQ 3b: More salient for eighth than sixth? Is the} pattern of mean level differences in the four developmental resources as a function of academic resilience group similar but more pronounced for eighthgrade students than for sixth-grade students?

RQ 3c: Eighth significantly more salient? Are differences in mean levels of the developmental assets between academic resilience groups greater for eighth graders than for sixth graders, as shown by a significant grade by motivational resilience group interaction term?

Note: For RQ 3, for eighth graders, gender was included as a CV. For sixth graders, gender showed no significant effect and was not included as a CV.
Partial: Three resources (autonomy, teacher support, and garden engagement) differed based on academic resilience group for eighth graders.

\begin{tabular}{lllll}
\multicolumn{1}{c}{ Aut. } & Tch. Sup. & Peer Sup. & Gard.Eng. \\
\cline { 2 - 4 } $8^{\text {th }}$ & Genabling) & -- & -- & -- \\
& -- & General & -- & General \\
$6^{\text {th }}$ & -- & -- & -- & -- \\
\hline & -- & -- & -- & -- \\
& -- & -- & -- \\
\hline
\end{tabular}
significant re was only evidence that the resources were important for eighth-grade academic resilience.

No. The grade*resilience group interaction term was not significant. 


\section{Research Ouestion 4:}

Do levels of the four

developmental resources

(autonomy, teacher

support, peer support,

and garden engagement)

differ as a function of

dual resilience group? Dual resilience.

RQ 4a: Eighth graders. For eighth-grade students, do the mean levels of the four developmental resources (autonomy, teacher support, peer support, and garden engagement) differ as a function of dual resilience group?

RQ 4b: Eighth more salient than sixth? Is the pattern of mean level differences in the four developmental resources as a function of dual resilience group similar but more pronounced for eighth-grade students than for sixth-grade students?

\section{RQ 4c: Eighth significantly more salient? Are}

differences in mean levels of the developmental assets

between dual resilience groups greater for eighth graders

than for sixth graders, as shown by a significant grade by

motivational resilience group interaction term?

Note: For RQ 4, for eighth graders, the effect of gender appeared to vary by resilience group and thus gender was included as an IV instead of a $C V$, with significant gender effects for autonomy, teacher support (marginally), and peer support. For sixth graders, gender showed no significant effect and was not included as a CV.

\section{Research Question 5:}

Predicting dual

\section{resilience.}

Do each of the

developmental resources

predict dual resilience

group membership?
Partial: Three resources (autonomy, teacher support

resilience group for eighth graders.

\begin{tabular}{|c|c|c|c|}
\hline Aut. & Tch. Sup. & Peer Sup. & Gard.Eng. \\
\hline (Enabling) & (Enabling) & -- & -- \\
\hline General & General & -- & General \\
\hline Protective & Protective & -- & -- \\
\hline- & Enabling & -- & Enabling \\
\hline General & General & -- & General \\
\hline Protective & Protective & -- & -- \\
\hline
\end{tabular}

No: Pattern is similar for sixth graders, and is not more pronounced for eighth graders than for sixth.

No. The grade*resilience group interaction term was not significant. and garden engagement) differed based on dual

\section{RQ 5a1: Unique effects for eighth graders. Do each of Partial: Only autonomy uniquely predicted dual} the developmental resources, with special focus on resilience group for eighth graders. autonomy and garden engagement, uniquely predict dual resilience group membership for eighth graders after controlling for all other developmental resources?

\section{RQ 5a2: Unique effects for eighth more salient than}

sixth? Are the four developmental resources

descriptively stronger unique predictors of dual resilience group membership for eighth graders than for sixth graders?

\begin{tabular}{lllll} 
th & Aut. & Tch. Sup. & Peer Sup. & Gard.Eng. \\
\cline { 2 - 5 } $8^{\text {Enabling }}$ & -- & -- & -- \\
& Protective & -- & -- & -- \\
\hline $6^{\text {th }}$ & (Enabling) & -- & -- & -- \\
& (Protective) & Protective & -- & Protective
\end{tabular}

No/Partial: Although autonomy seemed to be a more salient unique predictor for eighth graders than for sixth graders, teacher support and garden engagement were stronger predictors for sixth graders. 


\section{Research Question 5} cont'd: Predicting dual resilience.

Do each of the developmental resources predict dual resilience group membership?
RQ 5a3: Unique effects for eighth significantly different than sixth? Do the four developmental resources differ significantly as unique predictors of dual resilience group membership for eighth graders in comparison to sixth graders?

Note: All models for 5 a controlled for gender Gende Note: All models for 5 a controlled for gender. Gender was not a significant unique predicto
the eighth-grade model, but was a significant unique predictor in the sixth-grade model.

RQ 5b1: Autonomy as mediator for eighth graders. Does autonomy mediate the relationships between the three remaining developmental resources (teacher support, peer support, and garden engagement) and dual resilience group membership for eighth graders?

\section{RQ 5b2: Autonomy stronger mediator for eighth than} sixth? Is autonomy a descriptively stronger mediator of the relationships between the remaining developmental resources and dual resilience group for eighth graders than it is for sixth graders? support. graders.
No/Partial. Although the regression model for eighth graders significantly differed from that for sixth

graders, the difference was due not to regression paths

but in the correlation between autonomy and teacher

Partial: Autonomy appeared to at least partially mediate the relationship between teacher support and dual resilience group, but not that between garden engagement and dual resilience group, for eighth

\begin{tabular}{cllll}
\hline \multirow{2}{*}{$8^{\text {th }}$} & Aut. & Tch. Sup. & Peer Sup. & Gard.Eng. \\
\cline { 2 - 5 } & n/a & Full & n/a & Not mediated \\
& Partial & n/a & n/a \\
\multirow{2}{*}{$6^{\text {th }}$} & n/a & Partial & n/a & Full \\
& $n$ & Partial & $n / a$ & Partial \\
\hline
\end{tabular}

Partial: Autonomy seemed to be a stronger mediator for teacher support for eighth graders, but was a stronger mediator of garden engagement for eighth graders.

Note: Models for $5 b$ included gender when gender was significant in $5 b$ for that resource. For eighth graders, gender was included for the models for teacher support and garden engagement as enabling resources. For sixth grade, gender was included in the models for teacher support and garden engagement as protective resources. Gender appeared to be at least partially mediated by autonomy for eighth graders. Autonomy fully mediated the (marginally significant) relationship between gender and dual resilience group in the model for garden engagement as enabling resource, and reduced the strength of the gender effect in the model for teacher support as an enabling resource. Gender was not mediated by autonomy for sixth graders. 
ANOVAs: dual resilience groups. Differences between eighth-grade dual resilience groups in the analyses of variance followed a similar pattern as those found for motivational resilience groups. Autonomy and teacher support showed the clearest differences, whereas differences for garden engagement were less pronounced. Peer support did not differ significantly as a function of dual resilience group. The same approximate pattern of results was seen for sixth graders, and the grade by group interaction term in the combined sample was not significant, again providing no evidence that resources were significantly more salient for eighth graders.

\section{Regressions: unique predictors of dual resilience group. A multi-group} regression showed that, for eighth graders, only autonomy uniquely predicted dual resilience group membership after controlling for the other resources. For sixth graders, in contrast, autonomy (marginally), teacher support, and garden engagement all predicted dual resilience group. Invariance testing showed that regression paths did not differ significantly between the grades. However, the eighth- and sixth-grade models did differ significantly, a difference apparently due to different strengths for the correlation between teacher support and autonomy between the grades.

\section{Regressions: autonomy mediating prediction of dual resilience group.}

Finally, meditational analyses tested whether autonomy mediated the relationships between the developmental resources and dual resilience group whenever a significant relationship was present. For eighth graders, autonomy appeared to fully mediate teacher support in its role as an enabling factor (being in the resilient vs. the average group) for dual resilience, and to partially mediate teacher support as a protective factor 
(being in the average vs. the stress-affected group). The role of garden engagement as a (marginal) enabling factor was not mediated by autonomy. For the sixth graders, there were two differences in mediation patterns: autonomy only partially mediated the enabling role of teacher support, and autonomy did seem to mediate the role of garden engagement.

\section{Strengths, Limitations and Future Studies}

The conceptualization, measurement, design, and generalizability of this study each bring particular strengths, which we discuss below. This study is a first step in the exploration of a new concept, and has several limitations. One of the most notable limitations lies in the problem of examining a developmental process at one time point. These limitations, in and of themselves, offer further insight to the concepts of motivation, resilience, and development, and we have selected some of the most compelling to discuss here.

\section{Conceptualization}

This study is strengthened by using stage-environment fit, self-determination theory (SDT), and resilience--all powerful frameworks--in combination to propagate a new conception of developmentally-calibrated motivational resilience. Each theoretical approach addresses an important (and mutually-complementary) swathe of the literature on how to support students' success. Self-determination theory offers engagement as a powerful measure of student success, and offers a robust context-based model for fostering motivation. Stage-environment fit and resilience frameworks, in-turn, provide big-picture explanations of why motivation is important to students. In this study, 
however, we omitted certain aspects of the contexts and self-systems in SDT that would be useful to include in future studies. We examined only the contexts found within schools, but students' families remain a key motivational context even as students age. Likewise, we focused on autonomy as a key self-system process for early adolescents, but competence and belonging are inextricably linked with autonomy in students' motivational development and development of healthy academic identities.

Stage-environment fit, in turn, addresses the need to understand developmental changes in motivation, and guides an understanding of which parts of students' motivational systems are particularly salient in early adolescence. Self-determination theory, while offering powerful motivational constructs, lacks this developmentallycalibrated approach, and its measures do not capture some of the important aspects of adolescent motivation. Most palpably lacking from SDT, in this study, was an ability to directly measure identity, which many argue is the essential organizing force in adolescent development. Processes of motivational resilience or stress-affectedness in school are, to a large extent, a set of healthy identity development challenges that students must negotiate. SDT simply does not have the tools to address the richness of identity. Using measures from multiple theories to propagate the resilience framework in future studies would give a richer and more direct picture of the adolescent experience.

Additionally, this study focused on the developmental processes of at-risk students in a school setting, and did not address other closely-related (and interdependent) aspects of their identity development and resilient adaptation to risk. 
Students' holistic socio-emotional development has emerged as a promising area of research (Roeser \& Peck, 2003), encouraging educational practices that support students' selves as well as academic outcomes. For example, future studies exploring the risk of "eighth-gradeness" would benefit from including student perceptions of stress and emotional well-being.

Additionally, future studies studying diverse and at-risk students would benefit from exploring the developmental processes that underlie the impacts of culture and diversity as well as discrimination and poverty. Ethnic group and poverty are both relatively well-explored demographic factors in their relation to student academic success, but ethnicity, poverty, and other cultural factors are often confounded. In this sample, African students outnumbered African-American students, Southeast Asian refugee families outnumbered financially stable East Asian families (whose students traditionally perform well in comparison to other ethnic groups), and Caucasian students were largely composed of Central European and Russian refugees. Exploration of identity in samples such as this one could permit an investigation of how to disentangle ethnicity, nationality, poverty, and immigration status as they relate to student resilience.

\section{Measurement}

The measures of engagement, autonomy, and teacher support in this study provided a stable empirical foundation to balance this study's exploratory nature. Similar forms of these items have been used for two decades; they have been validated in several samples and tend to display strong measurement properties and predictive 
power. Because similar scales have been widely used in already-collected data sets and continue to be used, many aspects of this study could be easily replicated. Additionally, combining engagement data with achievement data gives a more meaningful outcome measure. However, future studies would benefit from additional or modified measures.

For example, for our measure of autonomy, we selected the autonomous (identified) self-regulation component of the larger self-determined motivation construct, but it could be useful to examine this component alongside students' reports of the remaining three motivation styles (intrinsic, introjected, and external.) Measures of alternative conceptualizations of autonomy could also be helpful. Additionally, this study relied on an examination of students' engagement with garden-based education as an activity that can be purposeful, but future studies could assess students' actual perceptions of purposeful participation. This study measured students' perceptions of peer support, but future studies would benefit from also measuring the extent to which students were supported by peers academically. This study used student ratings, but multiple reporters (e.g. teachers, parents, or observers) would help the validity of future studies. Finally, this study used measures that were valid for both sixth and eighth graders, but future studies could assess factor structure as well as examining particular subscales or individual item responses to see whether there were grade (or gender) differences in what it was about each construct that was salient for students. In particular, it would be helpful to separately examine the emotional and behavioral components of both engagement and disaffection.

\section{Design}


Researchers agree that developmental designs are more appropriate than singletime-point designs when assessing developmental processes (such as resilience), and recent strides in methodology permit enriching analyses of developmental data, crosssectional designs persist by virtue of their simplicity. We elected to use a crosssectional design in this study for two practical reasons related to the available data. First, it allowed us to include the construct garden-based education, which both provided needed quantitative research on garden-based education and offered a means by which to explore (potentially) purposeful and authentic learning experiences.

Second, this cross-sectional approach allowed a cautious and timely first step in the process of investigating developmentally-calibrated motivational resilience. This study is an initial venture with a new conceptualization of resilience, and using a crosssectional design to explore this concept allowed us to test the waters, capturing a snapshot of early adolescent motivational resilience processes that can guide future research. At the same time, the non-developmental nature of this design was the major limitation of the study, and the next step in this research is to observe resilience processes directly. Future studies could measure individual students' trajectories as they progress through middle school as well as measuring students' motivation and achievement before the transition into middle school and after the transition into high school. This study does not address resilience in its nature as a process, rather than a static occurrence, and does not have information on how high motivation and achievement need to be for students to truly be resilient to academic risk, but multipletime-points designs could measure resilience directly. 
Finally, future research would benefit from a more person-centered approach. The current study used a variable-centered approach to investigate students' developmentally-calibrated resilience as defined in their relation to our chosen constructs. One key finding in this study, however, was that the processes that supported motivation and achievement seemed to differ for boys and girls as well as for sixth- and eighth-grade students. Future studies could use profile analyses or other person-centered designs to examine the different motivational pathways taken by different groups of students.

\section{Generalizability}

One major strength of this study was its diverse sample, which addresses a need for understanding motivational processes in demographic at-risk students. However, a major limitation of this this study was its use of only one cohort of eighth- and sixthgraders and only one school. The sixth graders' levels of engagement and achievement, used as a yardstick for resilience in this study, are subject to cohort effects, as are all all processes observed in the eighth graders. Communication from school personnel at the time of study and initial analyses within multiple years of data gave no reason to suspect such an effect, but this school might well have offered a unique motivational environment that limits generalizability. For example, it was the only school in that time period to move from a failing to a passing grade on state report cards, benefitted from collaboration with a university in its gardening project, and school culture had an explicit focus on attending college. Future studies would benefit from a larger sample using multiple schools. 


\section{Third Variables}

In this study, several unmeasured variables could potentially provide alternate explanations for the observed relationships among variables. Of these, poverty serves a driving role. Socioeconomic status is known to impact students' academic lives in drastic and consistent ways. Poverty can contribute to chronic stress and inadequate nutrition that hampers students' abilities to engage and achieve in school (Heckman, 2007.) Students from low-income immigrant families might have more familiar responsibilities that leave less time for homework, and cultural differences could make relating with teachers and peers more difficult. In these examples, one could see how a student could present low teacher support, low peer support, low engagement, and low achievement without any particular causal links between those phenomena. Future studies would benefit from directly assessing students' individual demographic risk factors in order to examine their role in students' motivational and academic resilience.

\section{Implications}

This study was concerned with understanding the nature of eighth graders' motivational and academic resilience. The findings offer a greater understanding of eighth-grade as a time of motivational and academic risk, when combined with demographic risk factors and traditional middle schools. They also paint a picture of the resources that are important for eighth-grade students' motivational and academic resilience, and whether those resources are more important (or, more often, not) for eighth graders than for sixth graders. These findings also permit an understanding of the relationships among motivational and academic resources, and how the way they 
collectively shape students' motivation and achievement may differ for eighth graders and sixth graders. This study also explored the developmental calibration of academic risk and resilience. It offers a new conceptualization of academic risk and illustrates the developmental salience of different motivational and academic resources. Finally, this study demonstrated the need for a more salient and nuanced consideration of the role of gender in motivational and academic resilience.

\section{"Eighth-gradeness"}

Eighth grade as a time of risk and opportunity. One primary goal of this study was to explore eighth-grade as a critical window of both educational risk and opportunity, when students have new needs and capacities but just before their educational trajectories tend to stabilize for the worse, and the findings seemed to support that story. Eighth graders' achievement and motivation were moderate: mean levels of both GPA and engagement were above the midpoint of the scales. Even considering the overall context of risk posed to these students by demographic factors and a traditional middle school setting, they still reported being more engaged than not and were usually passing their classes. As expected, however, the eighth graders did have significantly lower engagement and achievement than sixth graders in the same traditional middle school setting and same context of demographic risk. The conjunction of finding both lower levels for eighth graders and finding that education outcomes were still relatively positive is notable: When considering eighth grade as a time of both risk and opportunity, capitalizing on the opportunities of "eighthgraderness," is much more difficult if students are already failing and checked-out. 
These findings, then, provide support for the idea of eighth grade as a potential critical window for at-risk students.

\section{Preliminary Results: Resources for Eighth-grade Motivation and Achievement}

Eighth graders also had moderate (above the midpoint) levels of each of the four developmental resources. This was not surprising: if autonomy, teacher support, peer support, and garden engagement serve as resources for students' motivation, it would follow that students' perceptions of having these resources would accompany their moderate levels of outcomes.

Developmental resources for motivation. Correlations between each of the four developmental resources and engagement showed that all four developmental resources appeared to be resources for eighth-grade motivation. For eighth graders, as expected, autonomy was correlated quite strongly with engagement. Unexpectedly, though, correlations did not show that autonomy was a more salient motivational resource for eighth graders than for sixth graders. While we expected autonomy to be important for sixth graders, the association for sixth graders seemed to be slightly stronger for sixth graders than the (already very strong) association for eighth graders.

As expected, teacher support was also strongly related to engagement. At the same time, the association with teacher support was, as with most associations, slightly higher for sixth graders than for eighth graders. Additionally, because levels of teacher support remained very high from sixth grade to eighth grade, the general picture begins to emerge that teacher support is an important motivational resource for all students, but may not serve a particularly different role for eighth graders than for sixth graders. 
Peer support was moderately associated with engagement, as expected indicating that peer support seemed to serve as a resource for motivation.

(Unexpectedly, the association between peer support and engagement in eighth graders seemed to hold only for the eighth-grade boys, a finding which will be discussed later.) However, because eighth graders reported quite high mean levels, and because nearly all students (as indicated by very high kurtosis) reported these very high levels peer support, it may be that peer support is a motivational resource which is being largely already met for these students. While mean levels did not differ between sixth and eighth graders, peer support was the only resource for which, as predicted, correlations were stronger for eighth graders than for sixth graders (who showed no significant association.)

Garden engagement correlated with engagement for eighth graders, as expected, but much more weakly than was expected. Thus, garden engagement seemed to be a resource for motivation, but not a very powerful one. Moreover, this association seemed to be quite a bit weaker for eighth graders than for sixth graders, which was surprising.

Associations with achievement. Autonomy, teacher support, and garden engagement all showed fairly uniform weak correlations with GPA. Contrary to expectations, peer support was not correlated with GPA. While not surprising, these weak (and absent) correlations inform the picture of academic resilience that associations were weak. This could be due in part to common method variance, in that engagement and all four of the developmental resources were assessed via students' 
self-reports of their perceived experiences, while GPA is a formal assessment of achievement made by a variety of teachers. Additionally, the SSMMD posits that motivational resources influence achievement through engagement. That is, although engagement is generally a strong predictor of GPA, GPA is a more-removed outcome, and is likely harder to influence than engagement. Even if students feel more (or less) motivated, they may still have a hard (or easy) time taking tests or understanding how to be successful in coursework.

While we expected that autonomy, teacher support, and garden engagement might be better resources for eighth-grade achievement than was peer support, we did not expect that GPA and peer support would be completely uncorrelated for eighth graders. This finding, however, does fit with our understanding of the importance of peers to educational success. Whereas having supportive friends might make one feel more emotionally invested in school, regardless of whether those friends are scholastically oriented, the extent to which friends would help one get better grades would depend more on other factors (e.g. the academic engagement of those peers) than their supportiveness.

\section{Resilience Groups: Resources for Motivational and Academic Resilience}

Resources for eighth-grade motivational resilience. As would be expected, the extent to which levels of the developmental resources differed based on students motivational resilience status followed a similar pattern as that shown in the mean levels and correlations. All four resources emerged as motivational resources when testing differences in resource levels among motivationally stressed, average, and 
resilient groups. Autonomy and teacher support appeared to be stronger resources and peer support and garden engagement appeared to be weaker resources.

Motivational resilience in eighth grade versus sixth grade. We expected that these resources would be more salient resources for eighth grade and thus would show more pronounced differences among eighth-grade resilience groups than among sixthgrade resilience groups. As indicated by the resources' correlations with engagement, however, the resources appeared to be just as strong for sixth graders as for eighth graders, with the exception of peer support, which was only a resource for eighth graders. Thus, we can conclude that autonomy, teacher support, peer support, and garden engagement all appear to be resources for eighth-grade motivation, and that autonomy, teacher support, and garden engagement are also resources for sixth-grade motivation.

Resources for eighth-grade academic resilience. As would be expected from correlations, levels of the four developmental resources differed to a much smaller degree among academic resilience groups. Autonomy, teacher support, and garden engagement all emerged as resources for students' academic resilience, but differences between groups were smaller, and generally only the differences between the resilient and stress-affected academic resilience groups were significant. Again, this is unsurprising, given the low strength of the correlations, but did show that these three resources were, however weakly, still apparent academic resources for eighth graders. Peer support showed no significant differences in levels among the groups, as would be expected given the lack of correlation between peer support and GPA. 
Academic resilience in eighth grade versus sixth grade. We hypothesized that the developmental resources would be more salient resources for eighth graders' academic resilience than for sixth graders. Testing this hypothesis formally showed no significant interaction between grade and resilience group, which is consistent with the similarity of correlations between sixth and eighth graders. Differences in levels of resources between the groups, while similar in nature and slight for both groups, were only significant for eighth graders. That is, autonomy, teacher support, and garden engagement did appear to be important resources for eighth-grade academic resilience and, if not unrelated to sixth-grade resilience, at least did not emerge as clearly as academic resources for sixth graders. This could be due to increased salience of the resources for eighth graders: perhaps the resources impacted both grades' motivation but this increased motivation only translated into increased grades for eighth graders. Perhaps, for example, a sense of autonomy over one's schoolwork helped sixth graders motivationally, but for eighth graders also translated into a sense of ownership over one's academic performance, making it a higher priority for students to be academically successful. It is also possible, however, that the finding of differences for eighth graders and not for sixth graders is an artifact of the ways the groups were constructed: because eighth graders had fewer resilient students than did sixth graders, those students who were in the eighth-grade academic resilient group may have had more extreme scores when compared with the students in the stress-affected group.

Resources for eighth-grade dual resilience. The four resources functioned very similarly in regard to eighth-grade dual resilience groups as they did for eighth-grade 
motivational resilience groups. As they did with eighth-grade motivational resilience groups, the resources seemed to serve a similar role for sixth graders as for eighth graders, which was counter to our expectations but not surprising given the correlations and the pattern of results for motivational resilience. The exception was peer support, which did not emerge as a resource for eighth-grade dual resilience, which was unsurprising given the very slight differences among eighth-grade peer support scores and the lack of correlation of peer support with GPA. As with motivational resilience and academic resilience groups, however, levels of autonomy, teacher support, and garden engagement did differ among eighth-grade dual resilience groups. Results did not differ between eighth-and sixth-grade resilience groups.

As with motivational resilience, differences in levels of autonomy were very pronounced between eighth-grade dual resilience groups, and (in both the ANOVA results and the separate regression analyses from RQ5b) appeared to serve both an enabling and a protective role. Similarly, differences in levels of teacher support were less pronounced than those in autonomy among dual resilience groups, but teacher support still appeared to serve both an enabling and protective role (likewise in both ANOVA and regression analyses). As was shown in analyses for motivational resilience, the dual resilience ANOVA results showed that levels of garden engagement differed between eighth-grade dually resilient and dually stress-affected groups, and the dual resilience regression analysis showed that garden engagement played a marginally significant enabling role.

The finding that dual resilience groups followed the pattern shown by 
motivational resilience groups makes sense considering the primacy of engagement as an educational outcome and that achievement is modeled as a more distant outcome. If the dual resilience groups are conceptualized as taking subsets from the motivational resilient and motivationally stressed groups of those students with the most extreme high or low grades, one would expect that differences would correspond to those found in motivational groups and just be a little more pronounced, which is what we found. In all, we found that, when considering eighth graders' dual motivational and academic resilience, autonomy and teacher support (especially) garden engagement (to a lesser extent) appeared to be important resources $8^{\text {th }}$ grade motivation and achievement--but not more so than for $6^{\text {th }}$ grade motivation and achievement.

\section{Unique Effects and Mediation: Autonomy as a Driving Developmental Resource for Motivational and Academic Resilience.}

We were particularly interested in autonomy as a developmental resource for eighth-graders because we believed it might serve as a means by which teacher support, peer support, and garden engagement impact motivation and achievement. In this study, autonomy is students' identified motivation in regard to their academic experience- - the extent to which students do their schoolwork because it is personally important for them (instead of, for example, because they want to avoid getting in trouble.) In this sense, autonomy seems to capture some of the increasing sense of ownership students might feel (or have a heightened awareness of not feeling) over their school experience as they progress into adolescence. For sixth-grade students, in contrast, we thought that autonomy would still be an important resource, but that their relationships with their 
teachers might serve a more salient role than did their personal sense of academic ownership.

Autonomy's role for sixth graders. Autonomy did, indeed, seem to serve a central role for eighth graders in that it was the only unique predictor of eighth-grade dual resilience group membership. It is worth noting that, in the regression analyses, we focused on the resources as enabling or protective factors and did not investigate the resources in their more easily detected role as "general resources" that would predicting students' membership in the resilient instead of stress-affected group. That is, if we had investigated the general resource role we might have also found unique effects for other resources. As it was, however, autonomy was the only resource that predicted eighthgrade students resilient (vs. average) or stress-affected (vs. average) group membership when controlling for the other resources and for gender. The story that emerges is that, when considering how to boost students into resilience or out of risk, a) it appears to be more difficult to do for eighth graders than for sixth graders (for whom there were several unique predictors of resilience group) and, b) that in that hard-to-influence motivational picture for eighth-graders, autonomy emerged as the one most powerful resource for enabling and protecting students motivation and achievement.

For the sixth-graders, in contrast, autonomy was only a marginally significant unique predictor of dual resilience group. Teacher support and garden engagement also uniquely predicted dual resilience group, when controlling for the other resources. For sixth graders moreover, teacher support appeared to show a stronger unique effect than autonomy: each unit increase in teacher support corresponded to students being 4.58 
times more likely to be a member of the resilient group instead of the average group (as compared to 3.42 and 3.52 times more likely for autonomy, and garden engagement, respectively.) This is not to say that autonomy was not an important resource for sixth graders - the sixth-grade unique effect for autonomy was actually larger than that for the eighth graders - but autonomy did not appear to play a central role as a unique predictor of dual resilience group for sixth graders.

Autonomy as a mediator. The driving role of autonomy suggested in the unique effects, however, was less clear when examining the mediation analyses. Autonomy did, as expected, appear to be a stronger mediator of teacher support for eighth graders: teacher support (as both an enabling and protective factor) was at least partially mediated by autonomy for both eighth- and sixth-graders, but autonomy fully mediated teacher support only for eighth graders when teacher support was serving as an enabling factor. That is, it seemed that, for all students, one of routes by which teachers' provision of structure, warmth, and autonomy support helped motivation and achievement was through increasing students' sense of the coursework being important, which corresponds to our understanding of motivational processes. For eighth-graders, however, this provision of motivational support seemed to be only helpful for students if it resulted in their increased sense of importance and ownership over their schoolwork. This carries implications for understanding how teachers might be able to adjust the focus of their energies (e.g., providing more autonomy support) in order to best address the increasing motivational challenges faced by eighth graders.

Garden engagement, in contrast, was not significantly correlated with autonomy 
for eighth graders, and thus it followed that autonomy did not mediate the relationship between garden engagement and dual resilience group. Autonomy did, however, partially and fully mediate the role of garden engagement as an enabling and protective resource, respectively, for sixth graders. That is, for sixth graders, garden engagement did seem to help students' motivation and achievement by increasing their sense of the importance of school to them personally. Given the general pattern that garden engagement appeared to be a stronger resource for sixth graders than eighth graders in general, perhaps this indicates that purposeful and authentic learning experience can, as we expected, serve as a gateway to an increased overall sense of school being relevant—but only when those authentic learning experiences are a good fit with students' particular needs and interests. While garden engagement did not uniquely predict dual engagement group after controlling for all resources, it did still marginally predict dual resilience for eighth graders after controlling for autonomy alone. Perhaps, then, garden engagement is mediated by another pathway for eighth graders. For example, it could be that garden engagement was only a resource for students who had generally high teacher support and were able to maintain good relationships with the garden educators. Alternately, the less-structured and more-interactive format of garden education experience means that students often spend less time interacting with a teacher and more time working on projects with their peers. Accordingly, garden-based education might be especially stressful for students who perceive less peer support, and might serve as a pathway for students who are socially adept to translate that into academic success. 
The findings that autonomy might mediate the provision of meaningful coursework and teachers' motivational support in impacting student's overall academic success is consistent with a rich set of research exploring alternate measures of academic motivation. For example, Roeser, Eccles, and Sameroff (1998) found that teachers' support of relatedness, competence, and autonomy--including providing meaningful curricula--predicted increases in students" academic value, which, in turn, predicted increases in other important educational outcomes.

In future studies, collection of time-series would allow researchers to examine the dynamics among autonomy and other self-system processes that underlie resilience. A student's rating of autonomy as a stable characteristic twice a year can show associations with engagement or other assets, but not how associations occurred. By assessing students' time-specific perceptions over a span of time, researchers have begun to pinpoint the precise contextual assets associated with motivational outcomes and how motivational dynamics play out over time (e.g. Park et al. 2012). Looking at students' moment-to-moment reports of developmentally important motivational variables during key points in their academic trajectories could help pinpoint the "active ingredients" in students' academic and motivational resilience.

\section{Calibrating Existing Frameworks}

Although this study was designed with the specific goal of understanding eighth-grade motivation and academic success, a larger goal was to explore whether existing theoretical frameworks, could benefit from developmental calibration to the specific challenges and opportunities of early adolescence. That is, underlying the 
explicit investigation of how specific resources support eighth graders' motivation and achievement, this investigation was also guided by the intention to explore whether, consistent with stage-environment fit theory, resources we selected from selfdetermination theory and positive psychology might be more important for eighth graders than sixth graders.

Calibration of motivational resources. In this study we compared the selfdetermination approach to motivation, in which motivational needs are universal and important to students regardless of developmental period, to the stage-environment fit approach to motivation, in which some motivational needs are more salient for students as they enter particular developmental periods. Our findings were mixed: there was no clear evidence that any of the needs were particularly more important for eighth graders than sixth graders, but neither was there clear evidence that the processes of motivation for eighth- and sixth-graders were the same. Overall, we found that, in general, the same motivational resources present for both eighth- and sixth-grade students, but that differences were found in overall picture or gestalt of the eighth- and sixth-graders' processes of motivational support.

As expected autonomy and teacher support appeared to be the most important resources for both eighth graders and sixth graders, which is consistent with the current theoretical approaches for promoting educational success. The differences we did find lay mostly in apparent differences in processes involving autonomy and teacher support (which are discussed in the following sections.) In addition to looking at these processes over time, future studies would benefit from considering how constructs could be 
measured in a way that might better enable the detection of grade differences. Measures in the current study were explicitly designed to work well for all students, but perhaps, for example, certain items work better for older students than younger ones. It is likely that different aspects of teacher support are more important for students in different developmental periods, and a more detailed examination of autonomy could offer insight into what it is about autonomy that is helpful for students. Unlike teacher support and autonomy, peer support and garden engagement, did show differences in their importance between the grades. A consideration the reasons for these differences both informs an overall understanding of how to promote motivation and achievement and also offers future directions for the study of these constructs.

Calibration in regard to peer support. Despite serving only as a weak motivational resource, peer support was the only developmental resource that was clearly more important to eighth graders than to sixth graders. Unexpectedly, supplementary analyses showed that peer support was only a resource for male eighth graders. When analyzed separately, female eighth graders showed no association of peer support with engagement and no differences in levels of peer support among motivational resilience groups. As discussed, one reason for the lack of significant findings for peer support may have been the restriction in variance, in that nearly all students reported very high levels of peer support. Male eighth graders appeared to show a slightly lower mean level and a slightly larger variance than eighth-grade girls, so perhaps that slight increase in variation permitted the detection of more effects for peer support. The apparently large difference in peer support associations, however, 
between eighth-graders and sixth-graders and between eighth-grade boys and eighthgrade girls, suggest that peer support really was a more important resource for eight graders and for eighth-grade boys in particular.

Perhaps a sense of peer support was helpful for some aspect of sixth graders' and female students' well-being (e.g. mental health or social skills) that was not measured in this study, but this sense of peer support informed students' academic success only for male eighth graders. Future studies could more directly examining the routes by which peers could be a resource for students' motivation-for example, by measuring a student's peers' aggregated academic engagement, students ratings of peers commitment to school, or the extent to which students' peers help one another with classwork, for example_-it seems likely that peers would appear as a resource for all students. Even without considering the academic orientation of a student's peers, however, peer support still emerged as a resource for eighth-grade boys' motivation. It may be that older students, as a part of their overall identity formation, are more highly aware of the extent to which they feel valued and feel belonging among their peers, and this association indicated that this sense of support and acceptance among peers does seem to be more important for eighth graders. It would make sense, then that a sense of rejection or inability to find a social "home" at school could make students prone to being more emotionally or behaviorally checked out at school and thus serve as a liability to their educational success.

Gender differences in peer support. In considering why peer support was not also important for eighth-grade girls, it may be helpful to consider peer support in 
conjunction with the other resources. For eighth-grade girls, peer support not only lacked a significant correlation with engagement, it lacked significant correlations with any of the constructs in the study. For eighth-grade boys, in contrast, peer support demonstrated weak but positive correlations with both autonomy and garden engagement, and a moderately strong association $(r=.50)$ with teacher support. (Sixthgrade peer support associations were less consistent but were present for both boys and girls.)

For eighth-grade boys, then, higher levels of peer support seemed to correspond to an overall picture of motivational good news: those with higher peer support also tended to report higher teacher support, autonomy, and garden engagement. Perhaps male students relied more on peer support to foster the sense of commitment to school that would be required to relate positively with teachers, while female students tended to prioritize academics more than male students in a way that was independent of their perceived peer support. There may also be normative behavioral differences between eighth-grade boys and girls, in that girls might be more likely to have the higher social skills that aid relationships both with teachers and with peers, whereas boys might be more prone to behavioral and attentional difficulties that could make relationships more difficult.

Future research on peer dynamics for eighth graders could shed light on several additional possible explanations for gender differences in the role of eighth-grade peer support. For example, perhaps eighth-grade girls' peer support would correlate with other aspects of motivation if the sample included more girls who reported low peer 
support. Alternately, perhaps the construct of peer support as measured itself is biased, in that even acquaintance-level female adolescent relationships may be more characterized by emotionally supportive behaviors, at least superficially. Male adolescent relationships, in contrast, may be more characterized by shared activities than emotional support, and thus high social support for a male could demonstrate not simply friendship but an especially close friendship.

Calibration in regard to garden engagement. Counter to our expectations, garden engagement was the only construct that appeared to be a less important resource for eighth graders than for sixth graders. We hypothesized that the purposeful and authentic academic work inherent in garden-based education would make garden engagement a more salient resource for eighth graders. While it is possible this sense of purpose and authentic academic work was not actually important for eighth graders, it is also possible that these eighth graders did not actually experience their garden-based education as particularly purposeful or authentic. Conversations with garden educators at the school suggested that garden-based education for eighth graders was discontinued at this school because both the students and the educators seemed to find the experience frustrating. Mean levels indicated otherwise, in that the eighth graders reported moderate levels of garden engagement, but there was also a large degree of variance in these scores. Perhaps, then, there were some students for whom these gardening experiences served as a sought-after chance for a more holistic, active, and real experience at school, and another, more visible group of students for whom gardening did not. 
Informal focus groups with a different cohort of eighth graders at the same school suggested that there was a difference between activities that could seem authentic and purposeful only superficially (e.g., working hard to plant crops that would not be harvested until summer, when students were not at the school), and activities that actually capitalize on the eighth graders own particular interests (e.g., being able to cook and eat the food, having a role in selling produce as a part of a garden business). Additionally, these eighth-grade students were entering a stage of adolescence in which one's appearance and ability to fit in has a greater consequence for students (Eccles \& Midgley, 1989) and doing so as largely low-income students for whom buying clothing and other outward symbols of social status was a real struggle for families. While students talked about enjoying getting their hands dirty, they also expressed frustration with experience of being outside when it was often cold and wet, and a frustration with getting their hard-won clothing dirty.

Moreover, whereas younger students might be more likely to find simply being outside or increased physical activity to be intrinsically enjoyable, older students might not. Most of these students were experiencing poverty and living in impoverished neighborhoods. Eighth graders may have been facing more exposure to serious risk factors (such as gang involvement, drug use, and risky sexual behaviors) in addition to increased responsibility in their families, and outdoor activities might have seemed juvenile when compared to the very real challenges in the rest of their lives. When eighth-grade students in our focus groups talked about enjoying garden-based education, their enjoyment hinged largely on an experience of stress-release. Gardening 
was important to them not because it was purposeful but because they found the time interacting with the natural environment to be a rare and much-needed chance to relax. That is, whereas at-risk eighth graders might be averse to a garden-based education experience they find to be irrelevant or childish, it also seems that their experiences of risk might make gardening an even more helpful resource for them, if done well. An investigation of garden-based curricula (Skinner, Chi, \& the LEAG, 2011) found an abundance of garden education programs designed for younger students but almost no programs designed for eighth-grade students or above. Careful thought is needed about how, exactly, to make gardening an enriching experience for eighth graders.

Considering that we do not know whether garden-based education was experienced as purposeful and authentic by these students, future research would benefit from measuring students' sense of purpose directly. Whereas it is unlikely that most students would prefer inauthentic or irrelevant activities to purposeful ones, this preference would rely on the purposeful activities being well-designed and truly authentic. Purposeful learning activities might well be more helpful for older students and also be harder for teachers to successfully provide (when compared to more conventional activities) for older students than for younger students. True purposeful and authentic education would require a real interest in and commitment to building practices based on what is truly purposeful and authentic for adolescents--and on having the classroom and administrative contexts that would allow teachers to enact such practices.

Summary. In all, this study provided support for the self-determination theory 
view of motivation in that autonomy, teacher support, and garden engagement were all important for both eighth- and sixth-grade students. At the same time, there were enough differences between the grades that it seemed clear that it was important to at least investigate possible grade differences. That is, in addition to supporting selfdetermination theory, this study demonstrated the importance of also considering the stage-environment fit theory view that developmental stage can influence the salience of motivational needs.

Calibration of educational risk and resilience. In addition to exploring whether a focus on developmental calibration could assist the understanding of how to promote motivation, we were also interested in whether such calibration could assist the understanding of risk and resilience. A primary question in this study was whether students' developmental status (i.e., "eighth-gradeness") in a risky context could pose an additional source of risk. In addition to providing evidence that these eighth-graders did seem to experience greater academic risk than sixth graders, and exploring a developmentally-calibrated approach to measuring risk, this study raised some unexpected issues regarding eighth graders' educational risk and resilience. We hypothesized that both being older and being male would pose an educational risk that would manifest in lower mean levels of study constructs. In addition to mean level differences, however, we found unexpected evidence of risk in grade and gender differences in the processes among study constructs.

Developmentally-calibrated definition of resilience versus risk. This study explored a new potential method for investigating developmentally-dependent risk and 
resilience when longitudinal data are not available. Considering that eighth-graderness itself seemed to pose educational risk, pegging eighth-grade group cut-off values to those for sixth-graders in the same overall risky context (unaffected by "eighthgradeness") provided us with a preliminary and exploratory opportunity for investigating the relationships between the four developmental resources and the specific levels of engagement and achievement that would identify eighth graders who are doing at least as well as sixth graders with the same demographic risk factors and same middle school setting. Forming eighth-grade resilience groups using a more traditional approach, such as splitting eighth graders into thirds, would give information about which processes are at play for an eighth-grade student with engagement and achievement levels that would be considered good for an eighth grader, but would offer no means by which to consider adaptation to the risk posed by "eighth-graderness." (And, as widespread achievement gaps and high school dropout suggest, being good for an eighth grader might not be good enough.)

In lieu of being able to actually examine students' trajectories of resilience from sixth- to eighth-grade, this approach appeared successful as a preliminary method for exploring which processes are at play for eighth graders with levels of motivation and achievement that are not only good for an eighth grader, but also good for a sixth grader. That is, this approach provides an exploratory method for examining eighth graders who show not only resilient adaptation to the combined risk of demographic status, a traditional middle school, and developmental period, but resilient adaptation to the specific risk posed by eighth-graderness. Comparison of these results to those obtained 
in future longitudinal studies (see section on "Future Research") will be informative about the method used to form resilience groups in the current study.

The role of gender in processes of educational risk and resilience. The most obvious complication regarding gender arose in the formation of the resilience groups. As expected, because male students generally have lower motivation and achievement than female students, the resilience groups were not perfectly balanced by gender: there were more females than males in the resilient groups and more males than females in the stress-affected groups. This difference, while problematic for the clear interpretation of gender's relationship with resilience groups in the analyses, reflects an authentic gender difference in students' risk and resilience.

Just as being resilient only in comparison to other eighth graders might not reflect an actual adaptive level of resilience, being resilient "for a male" really is not the same thing as being resilient "for a female." Thus, we chose to form the resilience groups in regard to concrete levels of motivation and achievement instead of forming groups with equal numbers of boys and girls. This difference raised a concern for interpretation largely in regard to the eighth-grade dually resilient group, which only had three boys, in comparison to its nine girls. When analyzed separately in supplementary analyses, we observed that resources generally served an enabling role more often for girls, and a protective role more often for boys, and that this pattern held for motivational, academic, and dual resilience groups. This pattern could reflect a genuine difference in the role these resources played for students, or could reflect the fact that there were a greater number of girls in the resilient groups and boys in the 
stress-affected groups, thereby more easily allowing those groups to be distinguished by enabling or protective factors, respectively. Either way, it seems to be the case that these resources appeared to be better able to buffer eighth-grade-boys' academic risk than to promote their resilience. In particular example, whereas autonomy served an enabling role for female students' academic resilience, there were no factors that served an enabling role for male students' academic resilience.

The complications with gender were first apparent in the series of ANOVAs. For eighth-grade academic resilience, we were able to simply include gender as a covariate. For motivational and academic resilience, however, the relationship between gender and the developmental resources appeared to vary by resilience group, making it inappropriate to include gender as a covariate. Instead, gender was included as its own independent variable, alongside resilience group. There were significant gender differences in levels of autonomy, teacher support, and peer support. Although no interaction terms were significant at the multivariate level for the ANOVAs (and therefore these interactions should be interpreted only anecdotally), there were significant univariate gender-by-group interactions for garden engagement in both the eighth-grade motivational and dual resilience groups, and a significant univariate gender-by-group-by-grade interaction term for garden engagement in the combinedgrades ANOVA for eighth-grade dual resilience. In contrast, gender never showed a significant main effect at the multivariate level for sixth graders and there was, therefore, no basis for including gender even as a covariate in the sixth-grade ANOVAs for motivational, academic, or dual resilience. 
Autonomy as a mediator of gender effects. In order to control for gender in the regressions, we included gender as an additional IV predicting dual resilience group alongside the developmental resources. In these analyses, gender was a significant predictor of dual resilience in at least some of the models for both grades, as expected. Unexpectedly, however, gender appeared to be mediated by autonomy. Gender no longer uniquely predicted dual resilience group when controlling for autonomy, and, when explicitly testing autonomy as a mediator of teacher support and garden engagement controlling for gender, gender was unexpectedly also mediated in its relationship with dual resilience group.

Although a demographic factor like gender is not often conceptualized as a variable that would be mediated by a self-system belief, the finding does seems to make conceptual sense: One interpretation of this result might be that, when considering what it is about being a female (vs. male) eighth grader that seems to enable resilient motivation and achievement, the active ingredient in this "female-ness" for eighth graders is something about female students' identification with and ownership of their school experiences. This, indeed, corresponds to our general understanding of the changing academic identities of females and males as they move into middle adolescence (Simmons \& Blythe, 1998) in that it is more socially acceptable for girls to appear scholastically oriented, and generally less socially acceptable for boys to do so. This pattern was not evident for sixth graders: gender was still a significant predictor of dual resilience group after controlling for all developmental resources, and did not appear to be mediated when included as a control variable when testing autonomy as a 
mediator of teacher support or garden engagement. That is, in the regressions, gender appeared to be an important part of sixth-grade students' motivational and academic success, but there was no evidence that the relationship between gender and dual resilience group depended on students' academic identities — as there seemed to be for eighth-grade students.

Finally, gender showed an unexpected pattern among study constructs in that male students, in addition to tending to have lower mean levels, tended to show weaker correlations among constructs. Lower correlations were an unexpected, but prominent, component of eighth-grade students' educational risk in this study, as discussed below. The differences in associations among variables, however, serve primarily here as another example of how processes, in addition to levels, of risk and resilience seemed to differ for male and female students. For this reason, future studies would benefit from a person-centered that would enable a more in-depth investigation of male and female students' actual educational trajectories over time.

Strength of resources as a source of risk and resilience. We selected the four developmental resources because we thought they might be more important for eighth graders. We expected eighth graders to show somewhat stronger associations between the developmental resources than sixth graders, and that, correspondingly, the four developmental resources would emerge as stronger predictors of dual resilience for eighth graders. Instead, we found that correlations among nearly all study constructs (except those involving peer support) were descriptively stronger for sixth graders than for eighth graders. Accordingly, rather than resources being stronger predictors for 
eighth graders than for sixth graders, we saw that a) autonomy remained a strong predictor and that b) the other resources seemed to drop away. A major component of eighth-grade risk, then, might be that autonomy and other vital motivational resources start to lose some of their power to bolster eighth-graders' engagement.

Although motivation and achievement seem to stabilize after eighth grade, eighth-grade educational outcomes may already be less malleable than those for sixth graders. It might make sense that autonomy was the only remaining unique predictor of dually resilient or stress-affected group membership because sixth graders are more malleable and more amenable to being directly influenced by their contexts (specifically, here, by their teachers). That is, perhaps part of what is risky about being an eighth grader is an increased immunity to the positive effects of the other important motivational support. Eighth graders, in contrast, might need to have their contexts align with their own personal interests and identities in order to be susceptible to influence. In that way, autonomy may have increased in salience for eighth graders not in the actual strength of autonomy as a lever for motivational or academic change, but in that it is more necessary to facilitate the other resources.

\section{Implications for Promoting Eighth-grade Motivation and Achievement}

This study contributes to the current literature by providing a specific investigation of the factors that might promote at-risk eighth graders' motivation and achievement. As previously discussed, students' perceived autonomy and perceived teacher support emerged in this study as the most important factors for promoting eighth graders' educational success. These factors were important for both engagement and 
GPA, but, as expected, were much stronger resources for motivation. Autonomy, in particular, was consistently the strongest resource for motivation, as would be indicated by a very strong association with engagement. Because autonomy assesses students' experience of whether schoolwork is personally important to them, it should be part of the same overall process as their experience of working hard and being emotionally involved in the work. Additionally, because there was also quite a bit of variation in autonomy scores within eighth graders (unlike teacher support ratings, which were generally only high), autonomy seems to be a good candidate as a lever for motivational change simply because students tend not to already be at the ceiling of their autonomy perceptions.

Moreover, autonomy and teacher support appeared to be intimately connected in their role as resources for eighth-grade motivation and achievement. This was shown both in moderate correlations between autonomy and teacher support and in autonomy's role as a mediator of teacher support in predicting dual resilience. The patterns of relationships found in this study between teacher support, autonomy, engagement, and achievement correspond to the SSMMD, which posits that teacher support should be a proximal cause of autonomy along with other self-system processes, which should, in turn cause increased engagement, and, in turn, cause increased achievement.

When considering the relationship between autonomy and teacher support, the slightly weaker correlations between them for eighth graders could lead one to expect that the resources might emerge as more independent resources, as unique predictors of resilience, for eighth graders than for sixth graders. In this light, it was unexpected that 
autonomy and teacher support (along with garden engagement) were all unique predictors of dual resilience group for sixth graders, but that only autonomy was a unique predictor for eighth graders. It is worth noting that we only investigated unique predictors as enabling or protective factors, and that teacher support and garden engagement would likely also emerge as unique predictors of dually resilient versus dually stress-affected group membership. As it was, however, autonomy was the only resource to uniquely predict what might be considered more salient outcomes: preventing students from being in the stress-affected group, or boosting them into the resilient group.

Autonomy was not more important for eighth graders than sixth graders in the strength of effects; rather, it appeared to be the only resource to remain a strong predictor for eighth graders, whereas the other resources appeared to drop out. In this way, "eighth-gradeness" appeared particularly risky because there were fewer apparent mechanisms for promoting motivation or achievement. It appears, then that autonomy was not as sufficient as a motivational and academic resource for eighth graders as it was for sixth graders, but may have been more necessary.

Applications for schools. Middle school teachers know that teaching early adolescents can pose a particular challenge. The main implications of this study for middle school teachers are that a) while it may be more difficult to see a positive impact when providing support to eighth graders than sixth graders, the experience of having supportive teachers is still important for these students; and that b) their interactions with eighth graders may be more impactful to the extent that they can foster students' 
sense of ownership over their own school experience.

Fostering ownership. Autonomy, teacher support, and garden engagement all seemed to promote student motivation and achievement, and peer support seemed to promote student motivation for eighth-grade boys. It seems likely that different students might find different resources to be particularly important. Autonomy, however, seemed to be the primary resource for enabling or protecting students' combined motivation and achievement. That is, if teachers want to help students move into excellence or help students climb out of less-adaptive educational experiences, fostering students' perceptions of school being personally important to them seemed to be the key.

This, however, is likely not new information for teachers. A quarter-century ago, Eccles and Midgley (1989) argued that perhaps now that there was plentiful research on the importance of meet adolescents' needs for autonomy in school, practice would follow. Despite efforts, middle schools remain formal, controlling environments, likely because it is very difficult to successfully provide adolescents with autonomy in the classroom. The higher-order thinking skills and identity development that help spurt adolescents' desire for autonomy are only recently gained parts of their selves. Adolescents desire autonomy despite (or perhaps because of) their lacking experience exercising autonomy. Traditional middle schools do little to scaffold the ability to successfully be autonomous over one's school experience, likely because it is challenging and time-intensive to do so. However, in addition to helping meet adolescents' current developmental needs for autonomy, this kind scaffolding is likely vital for meeting early adolescents' needs to develop autonomy well during this key 
period. As one theorist explains, the adolescents' environments should meet "whatever structure he presently requires, but also view his present need ... on a developmental continuum along which growth towards independence and less need for structure is the long-term objective," (Hunt, 1975, p. 221).

Some suggestions for fostering ownership can be found in self-determination theory. Teachers can support student autonomy by both explaining how classroom activities are worthwhile, and relevant to students' own interests (Ryan \& Deci, 2009)-and also by choosing activities that really are worthwhile and relevant. In order to not only support autonomy but to do it authentically, (e.g. by giving students an opportunity for real decision-making and input), it might be important to focus on developing student's competence and strong relationships in conjunction with their sense of autonomy. These needs are complementary (Reeve, 2006), and fostering adolescents' needs in a way that strongly promotes autonomy, competence, and belonging in an interdependent way might be one way to both develop a sense of ownership and get students ready to take that ownership. In addition to providing real autonomy support, teachers can provide warmth that also conveys respect and trust, and provide structure that imparts the necessary information and skills for taking more responsibilities. In this way, teachers might help scaffold ownership by getting students ready for the experience of real investment and contribution of value to one's environment.

Research on students' sense of academic value both supports the idea that students' motivational support can be successful to the extent that it supports and identification with schoolwork, and offers suggestions on how to promote this sense of 
academic ownership. In one study, for example, Roeser, Eccles, and Sameroff (1998) found that teachers' support of autonomy, relatedness and competence all supported students' academic valuing. As they explain, supporting each of these three needs can promote academic identity in complex and developmentally-situated ways. For example, provisions of structure promote students' academic confidence and self-worth, which can protect adolescents' academic identity against disaffection, and that academic identity, in turn, can help students approach school with a sense of purpose and meaning that can further help adolescents' success.

Garden engagement. Garden engagement was not as strong a resource as expected, which highlights the importance of need for developmental attunement when designing garden-based education and other authentic classroom experience. Garden engagement was, however, a consistent resource for eighth graders that was not dependent on students' sense of academic ownership. This is one of the only studies to link garden engagement quantitatively to academic outcomes, so this is notable. Perhaps garden engagement and other out-of-classroom or purposeful academic experiences might be a way to promote the success of students whose sense of ownership is hard to bolster.

Peer support. Peer support was only a resource for eighth-grade boys' motivation, but the associations with all other motivational resources suggest that something about peer support was an important part of male eighth-graders' motivational success. It could be that supportive peer relationships are an important ingredient for students' sense of overall school commitment, or that students' social 
skills (or another factor underlying peer support) are an area of sensitivity that, when low, increase students' academic risk. Either way, healthy peer relationships seem connected to male eighth-graders' motivation. While one would hope that any student who seems isolated, rejected, or bullied would raise flags for teachers or administrators, the knowledge that poor relationships might impinge students' academic success could allow schools to make supporting these students a higher priority, given the many competing demands school personnel face. Schools could support students' capacity for supportive relationships by providing instruction or activities that would build students' interpersonal skills or emotional intelligence, or by providing opportunities for social connection via sports, clubs, or other organized school activities.

Conclusion. This study explored how best to enable and protect eighth-grade motivational and academic resilience. Although our developmentally-selected resources seemed to be, in general, equally important for sixth graders as for eighth graders, a few developmental patterns emerged. Autonomy seemed to be the driving resource for promoting eighth-grade resilience. Peer support was a resource for eighth-grade boys. Engagement in garden-based education was a resource for eighth graders, but was a stronger resource for sixth graders. Gender differences in relationships among variables were more pronounced than expected. Overall, this study served as a first step exploring a developmentally calibrated understanding of motivational resilience. We explored the intersection of stage-environment fit and self-determination theory within a resilience framework that permitted the examination of a new, developmentally-calibrated approach to measuring risk and resilience. In this process, we furthered the 
understanding of how to promote motivation and achievement for at-risk middle school students as they pass through the particular window of risk and opportunity in eighth grade. 


\section{REFERENCES}

Alspaugh, J. (1998). Achievement loss associated with the transition to middle school and high school. The Journal of Educational Research, 92(1), 20-25.

Anderman, E. M., \& Maehr, M. L. (1994). Motivation and schooling in the middle grades. Review of Educational Research, 64(2), 287-309.

Assor, A., Kaplan, H., \& Roth, G. (2002). Choice is good, but relevance is excellent: autonomy-enhancing and suppressing teacher behaviours predicting students' engagement in schoolwork. The British Journal of Educational Psychology, 72(Pt 2), 261-78.

Baron, R. M., \& Kenny, D. A. (1986). The moderator-mediator variable distinction in social psychological research: Conceptual, strategic, and statistical considerations.Journal of Personality and Social Psychology, 51(6), 1173-1182

Blackwell, L. S., Trzesniewski, K. H., \& Dweck, C. S. (2007). Implicit theories of intelligence predict achievement across an adolescent transition: A longitudinal study and an intervention. Child Development, 78(1), 246-63.

Blair, D. (2009). The child in the garden: An evaluative review of the benefits of school gardening. The Journal of Environmental Education, 40(2), 15-38. 
Bowker, R., \& Tearle, P. (2007). Gardening as a learning environment: A study of children's perceptions and understanding of school gardens as part of an international project. Learning Environments Research, 10(2), 83-100.

Brewster, A. B., \& Bowen, G. L. (2004). Teacher support and the school engagement of Latino middle and high school students at risk of school failure. Child and Adolescent Social Work Journal, 21(1), 47-67.

Bronfenbrenner, U., \& Morris, P. A. (1998). The ecology of developmental processes. In W. Damon \& R. M. Lerner (Eds.), Handbook of child psychology: Vol 1: Theoretical Models of Human Development (5th ed., pp. 993-1028). New York: Wiley.

Brule, H., \& Skinner, E. A. (2011). Motivational resilience during middle school : A developmentally-calibrated model of predictors of academic engagement. Poster Presented at the Society for Research on Adolescence, Vancouver, B.C., Canada.

Butler, A. C., \& Carr, L. (1968). Purpose in life through social action. The Journal of Social Psychology, 74(2), 243-250.

Chirkov, V. I. (2009). A cross-cultural analysis of autonomy in education: A selfdetermination theory perspective. Theory and Research in Education, 7(2), 253262. 
References

Connell, J. P., Spencer, M. B., \& Aber, J. L. (1994). Educational risk and resilience in African-American youth: Context, self, action, and outcomes in school. Child Development, 65(2), 493-506.

Connell, J. P., \& Wellborn, J. G. (1991). Competence, autonomy, and relatedness: a motivational analysis of self-system processes. In M. R. Gunnar \& A. L. Sroufe (Eds.), Self processes in Development: Minnesota Symposium on Child Psychology (pp. 43-77). Hillsdale, NJ: Lawrence Earlbaum Associates.

Côté, J. (2002). Comparing psychological and sociological approaches to identity: identity status, identity capital, and the individualization process. Journal of Adolescence, 25(6), 571-586.

Côté, J., \& Bynner, J. (2008). Changes in the transition to adulthood in the UK and Canada: The role of structure and agency in emerging adulthood. Journal of Youth Studies, 11(3), 251-268

Crosnoe, R., Johnson, M. K., \& Elder, G. H. (2004). Intergenerational bonding in school: The behavioral and contextual correlates of student-teacher relationships. Sociology of Education, 77(1), 60-81.

Damon, W. (2004). What is positive youth development? The Annals of the American Academy of Political and Social Science, 591(1), 13-24.

Damon, W. (2008). The path to purpose: Helping our children find their calling in life. New York: Simon and Schuster. 
Damon, W., Menon, J., \& Bronk, K. C. (2003). The development of purpose during adolescence. Applied Developmental Science, 7(3), 119-128.

Dawes, N. P., \& Larson, R. (2011). How youth get engaged: Grounded-theory research on motivational development in organized youth programs. Developmental Psychology, 47(1), 259-269.

Deci, E. L., Eghrari, H., Patrick, B. C., \& Leone, D. R. (1994). Facilitating internalization: The self-determination theory perspective. Journal of Personality, 62(1), 119-142.

Deci, E. L., \& Ryan, R. M. (1985). Intrinsic motivation and self-determination in human behavior. New York: Plenum Press.

Deci, E. L., \& Ryan, R. M. (2000). The "what" and "why" of goal pursuits: Human needs and the self-determination of behavior. Psychological Inquiry, 11(4), 227268.

Dirks, A. E., \& Orvis, K. (2005). An evaluation of the junior master gardener program in third grade classrooms. HortTechnology, 15, 443-447

Dornbusch, S. M., Erickson, K. G., Laird, J., \& Wong, C. A. (2001). The relation of family and school attachment to adolescent deviance in diverse groups and communities. Journal of Adolescent Research, 16(4), 396-422.

Eccles, J. S., Midgley, C., Wigfield, A., Buchanan, C. M., Reuman, D., Flanagan, C., \& Iver, D.M. (1993). Development during adolescence: The impact of stage- 
References

environment fit on young adolescents' experiences in schools and in families. The American Psychologist. 48(2), 90 - 101.

Eccles, J.S. \& Midgley, C. (1989). Stage-environment fit: Developmentally appropriate classrooms for young adolescents. In C. Ames \& R. Ames (Eds.), Research on motivation in education (pp. 139-186). London: Academic Press, Inc.

Eccles, J. S., Buchanan, C. M., Flanagan, C., Fuligni, A., Midgley, C., \& Yee, D. (1991). Control versus autonomy during early adolescence. Journal of Social Issues, 47(4), $53-68$.

Eccles, J. S., Flanagan, C., Lord, S., Midgley, C., Roeser, R., \& Yee, D. (1996). Schools, families, and early adolescents: what are we doing wrong and what can we do instead? Journal of Developmental and Behavioral Pediatrics, 17(4), 267276.

Eccles, J. S., Lord, S. E., Roeser, R. W., Barber, B. L., \& Jozefowicz Hernandez, D. M. (1997). The association of school transitions in early adolescence with developmental trajectories through high school. In J. Schulenberg, J. L. Maggs, \& K. Hurrelmann (Eds.), Health risks and developmental transitions during adolescence (pp. 283-320). New York: Cambridge University Press.

Eccles, J. S., Wigfield, A., \& Schiefele, U. (1998). Motivation to succeed. In W. Damon \& N. Eisenberg (Eds.), Handbook of child psychology, volume three: Social, emotional, and personality development (5th ed., pp. 1017 - 1095). Hoboken, NJ: John Wiley \& Sons. 
Erikson, E. H. (1968). Identity: Youth and crisis. New York: Norton.

Faul, F., Erdfelder, E., Lang, A.-G., \& Buchner, A. (2007). G*Power 3:

A flexible statistical power analysis program for the social, behavioral, and biomedical sciences. Behavior Research Methods, 39(2), 175-91.

Finn, J. D., \& Voelkl, K. E. (1993). School characteristics related to student engagement. Journal of Negro Education, 62(3), 249-268.

Finn, J. D., \& Zimmer, K. S. (2012). Student engagement: What is it? Why does it matter? In S. L. Christenson, A. L. Reschly, \& C. Wylie (Eds.), Handbook of research on student engagement (pp. 97-131). New York: Springer.

Fredricks, J. A, Blumenfeld, P. C., \& Paris, A. H. (2004). School engagement: Potential of the concept, state of the evidence. Review of Educational Research, 74(1), 59109.

Fry, P. S. (1998). The development of personal meaning and wisdom in adolescence: A reexamination of moderating and consolidating factors and influences. In The human quest for meaning: A handbook of psychological research and clinical applications (pp. 91-110).

Furrer, C., \& Skinner, E. A. (2003). Sense of relatedness as a factor in children's academic engagement and performance. Journal of Educational Psychology, $95(1), 148-162$. 
Furrer, C., \& Skinner, E. A., \& Pitzer, J. R. (2014). The influence of teacher and peer relationships on students' classroom engagement and everyday motivational resilience. National Society for the Study of Education, 113(1), 101-123

Fusco, D. (2001). Creating relevant science through urban planning and gardening. Journal of Research in Science Teaching, 38(8), 860-877.

Frankl, V. (1959). Man's search for meaning. New York: Simon \& Schuster.

Gottfried, A. E., \& Fleming, J. (2001). Continuity of academic intrinsic motivation from childhood through late adolescence: A longitudinal study. Journal of Educational Psychology, 93(1), 3-13.

Greene, J. P., \& Winters, M. A. (2006). Leaving Boys Behind: Public High School Graduation Rates. New York: Manhattan Institute for Policy Research.

Grolnick, W. S., \& Ryan, R. M. (1987). Autonomy in children's learning: An experimental and individual difference investigation. Journal of Personality and Social Psychology, 52(5), 890-898.

Grolnick, W. S., Kurowski, C. O., Dunlap, K. G., \& Hevey, C. (2000). Parental resources and the transition to junior high. Journal of Research on Adolescence, $10(4), 465-488$. 
Grolnick, W., Gurland, S. T., Jacob, K. F., \& Decourcey, W. (2002). The development of self-determination in middle childhood and adolescence. In J. S. Eccles \& A. Wigfield (Eds.), Development of achivement motivation (pp. 147-171). San Diego: Academic Press.

Herr, N. (1996). Mediation with Dichotomous Outcomes. Retrieved January 04, 2014, from http://www.nrhpsych.com/mediation/logmed.html

Hill, J. P. (1980). The family. In M. Johnson (Ed.), Toward adolescence: The middle school years. The seventy-ninth yearbook of the National Society for the Study of Education (pp. 32- 55). Chicago: University of Chicago Press.

Hill, P. L., \& Burrow, A. L. (2012). Viewing purpose through an Eriksonian lens. Identity, 12(1), 74-91.

Hill, P. L., Burrow, A. L., O’Dell, A. C., \& Thornton, M. A. (2010). Classifying adolescents' conceptions of purpose in life. The Journal of Positive Psychology, $5(6), 466-473$.

Horn, L., Chen, X., \& Adelman, C. (1998). Toward resiliency: At-risk students who make it to college. Washington, D.C.: U.S. Department of Education.

Hunt, D. E. (1975). Person-environment interactions: A challenge found wanting before it was tried. Review of Educational Research, 45, 209-230.

Hymel, S., Comfort, C., Schonert-Reichl, K., \& McDougall, P. (1996). Academic failure and school dropout: The influence of peers. J. Juvonen \& KR Wentzel 
(Eds.), Social motivation: Understanding children's school adjustment (pp. 313345). New York: Cambridge University Press.

Inhelder, B., \& Piaget, J. (1958). Adolescent thinking. In The growth of logical thinking: From childhood to adolescence. (pp. 334-350). New York: Basic Books. Isakson, K., \& Jarvis, P. (1999). The adjustment of adolescents during the transition into high school: A short-term longitudinal study. Journal of Youth and Adolescence, 28(1), 1-26.

Jang, H., Reeve, J., Ryan, R. M., \& Kim, A. (2009). Can self-determination theory explain what underlies the productive, satisfying learning experiences of collectivistically-oriented Korean students? Journal of Educational Psychology, 101(3), 644-661.

Jang, H., Reeve, J., \& Deci, E. L. (2010). Engaging students in learning activities: It is not autonomy support or structure but autonomy support and structure. Journal of Educational Psychology, 102(3), 588-600.

Janosz, M., Archambault, I., Morizot, J., \& Pagani, L. S. (2008). School engagement trajectories and their differential predictive relations to dropout. Journal of Social Issues, 64(1), 21-40.

Jimerson, S. R., Campos, E., \& Greif, J. L. (2003). Toward an understanding of definitions and measures of school engagement and related terms. The California School Psychologist, 8, 7-27. 
Kenny, M. E., Walsh-Blair, L. Y., Blustein, D. L., Bempechat, J., \& Seltzer, J. (2010). Achievement motivation among urban adolescents: Work hope, autonomy support, and achievement-related beliefs. Journal of Vocational Behavior, 77(2), 205-212.

Kiang, L., \& Fuligni, A. J. (2008). Purpose and meaning, ethnic identity, and adjustment among adolescents from Latin, Asian, and European American backgrounds. Journal of Youth and Adolescence, 39(11), 1253-1264.

Kilpatrick, W.H. (1918). The project method. Teacher's College Record, 19(4), 319 335.

Kindermann, T. A. (2007). Effects of naturally-existing peer groups on changes in academic engagement in a cohort of sixth graders. Child Development, 78(4), $1186-1203$.

Klem, A. M., \& Connell, J. P. (2004). Relationships matter: linking teacher support to student engagement and achievement. The Journal of School Health, 74(7), 26273.

Ladd, G. W., Herald-Brown, S. L., \& Kochel, K. P. (2009). Peers and motivation. In K. R. Wentzel \& A. Wigfield (Eds.), Handbook of motivation at school (pp. 323348). New York: Routledge.

Larson, W. (2000). Toward a psychology of positive youth development, American Psychologist, 55(1), 170-183. 
References

Lepper, M. R., Corpus, J. H., \& Iyengar, S. S. (2005). Intrinsic and extrinsic motivational orientations in the classroom: age differences and academic correlates. Journal of Educational Psychology, 97(2), 184-196.

Libbey, H. P. (2004). Measuring student relationships to school: attachment, bonding, connectedness, and engagement. The Journal of School Health, 74(7), 274-283.

Lieberman, G.A., and Hoody, L.L. (1998) Closing the Achievement Gap: Using the Environment as an Integrating Context for Learning. San Diego, CA: State Education and Environment Roundtable.

Luthar, S. S. (2006). Resilience in development: A synthesis of research across five decades. In D. Ciccetti \& D. J. Cohen (Eds.), Developmental psychopathology (2nd ed., pp. 739 - 795). Hoboken, NJ: John Wiley \& Sons, Inc.

Mackinnon, D. P., \& Dwyer, J. H. (1993). Estimating mediated effects in prevention studies. Evaluation Review, 17(2), 144-158.

Marchand, G. C. (2007). Developing a sense of academic ownership: A longitudinal analysis (Unpublished doctoral dissertation). Portland State University, Portland, OR.

Marchant, G. J., Paulson, S. E., \& Rothlisberg, B. A. (2001). Relations of middle school students' perceptions of family and school contexts with academic achievement. Psychology in the Schools, 38(6), 505-519. 
Marcoulides, G. A., Gottfried, A. E., Gottfried, A. W., \& Oliver, P. H. (2008). A latent transition analysis of academic intrinsic motivation from childhood through adolescence. Educational Research and Evaluation, 14(5), 411-427.

Marks, H. M. (2011). Student engagement in instructional activity: Patterns in the elementary, middle, and high school years. Educational Research, 37(1), 153-184.

Masten, A. S. (2001). Ordinary magic: Resilience processes in development. American Psychologist, 56(3), 227-238.

McInerney, D. M., Roche, L. a., \& Marsh, H. W. (1997). Cultural perspectives on school motivation: The relevance and application of goal theory. American Educational Research Journal, 34(1), 207-236.

Morrison, G. M., \& Allen, M. (2007). Promoting student resilience in school contexts. Theory into Practice, 46(2), 162-169.

National Center for Education Statistics. (1999). College Access and Affordability: Findings from the Condition of Education 1998. Education Statistics Quarterly (Vol. 1). Washington, DC.

Newmann, F.M., Wehlage, G.G. \& Lamborn, S. (1992). The significance and sources of student engagement. In F. Newmann (Ed.), Student engagement and achievement in American secondary schools (pp. 11-39). New York: Teachers College Press. 
Oyserman, D., \& Bybee, D. (2006). Possible selves and academic outcomes: How and when possible selves impel action. Journal of Personality and Social Psychology, $91(1), 188-204$.

Ozer, E. J. (2007). The effects of school gardens on students and schools: conceptualization and considerations for maximizing healthy development. Health Education \& Behavior: The Official Publication of the Society for Public Health Education, 34(6), 846-63.

Pekrun, R. (1993). Facets of adolescents' academic motivation: A longitudinal expectancy-value approach. In M. Maehr \& P. Pintrich (Eds.), Advances in motivation and achievement (pp. 139- 189). Greenwich, CT: JAI Press.

Ratcliffe, M. M., Merrigan, K. A, Rogers, B. L., \& Goldberg, J. P. (2011). The effects of school garden experiences on middle school-aged students' knowledge, attitudes, and behaviors associated with vegetable consumption. Health Promotion Practice, 12(1), 36-43.

Reeve, J. (2006). Extrinsic rewards and inner motivation. In C. M. Evertson \& C. S. Weinstein (Eds.),Handbook of classroom management: Research, practice, and contemporary issues (pp.645-664). Mahwah, NJ: Erlbaum.

Reeve, J., \& Jang, H. (2006). What teachers say and do to support students' autonomy during a learning activity. Journal of Educational Psychology, 98(1), 209 - 218. 
References

Reeve, J., Jang, H., Carrell, D., Jeon, S., \& Barch, J. (2004). Enhancing Students' Engagement by Increasing Teachers' Autonomy Support.

Roderick, M., \& Camburn, E. (1999). Risk and recovery from course failure in the early years of high school. American Educational Research Journal, 36(2), 303-343.

Roeser, R. W., Eccles, J. S., \& Sameroff, A. J. (2000). School as a context of early adolescents' academic and social-emotional development: A summary of research findings. The Elementary School Journal, 100(5), 443-471.

Roeser, R. W., Galloway, M., Casey-Cannon, S., Watson, C., Keller, L., \& Tan, E. (2008). Identity representations in patterns of school achievement and well-being among early adolescent girls: Variable- and person-centered approaches. The Journal of Early Adolescence, 28(1), 115-152.

Roeser, R. W., \& Peck, S. C. (2003). Patterns and pathways of educational achievement across adolescence: a holistic-developmental perspective. New Directions for Child and Adolescent Development, (101), 39-62.

Roeser, R. W., \& Peck, S. C. (2009). An education in awareness: Self, motivation, and self-regulated learning in contemplative perspective. Educational Psychologist, 44(2), 119-136.

Roorda, D. L., Koomen, H. M., Spilt, J. L., \& Oort, F. J. (2011). The influence of affective teacher-student relationships on students' school engagement and 
References

achievement a meta-analytic approach. Review of Educational Research, 81(4), 493-529.

Ryan, A. M. (2000). Peer groups as a context for the socialization of adolescents' motivation, engagement, and achievement in school. Educational Psychologist, $35(2), 101-111$.

Ryan, A. M., \& Patrick, H. (2001). The classroom social environment and changes in adolescents' motivation and engagement during middle school. American Educational Research Journal, 38(2), 437-460.

Ryan, R. M., \& Deci, E. (2009). Promoting self-determined school engagement. In K. Wentzel \& A. Wigfield, (Eds.), Handbook of motivation at school (pp. 171196).New York: Routledge.

Ryan, R. M., \& Deci, E. L. (2000). Self-determination theory and the facilitation of intrinsic motivation, social development, and well-being. The American Psychologist, 55(1), 68-78.

Ryan, R. M., Stiller, J. D. D. J., \& Lynch, J. H. H. (1994). Representations of relationships to teachers, parents, and friends as predictors of academic motivation and self-esteem. The Journal of Early Adolescence, 14(2), 226-249.

Skinner, E. A., \& Belmont, M. J. (1993). Motivation in the classroom: Reciprocal effects of teacher behavior and student engagement across the school year. Journal of Educational Psychology, 85(4), 571-581. 
Skinner, E. A., Chi, U., \& The Learning-Gardens Educational Assessment Group. (2012). Intrinsic motivation and engagement as "active ingredients" in gardenbased education: Examining models and measures derived from self-determination theory. The Journal of Environmental Education, 43(1), 16-36.

Skinner, E. A., Kindermann, T. A., Connell, J. P., \& Wellborn, J. G. (2009).

Engagement and disaffection as organizational constructs in the dynamics of motivational development. In K. Wentzel \& A. Wigfield, (Eds.), Handbook of motivation at school, (pp. 223 - 245.) New York: Routledge.

Skinner, E. A., Kindermann, T. A., \& Furrer, C. J. (2008). A motivational perspective on engagement and disaffection: Conceptualization and assessment of children's behavioral and emotional participation in academic activities in the classroom. Educational and Psychological Measurement, 69(3), 493-525.

Skinner, E. A., Wellborn, J. G., \& Connell, J. P. (1990). What it takes to do well in school and whether I've got it: A process model of perceived control and children's engagement and achievement in school. Journal of Educational Psychology, 82(1), $22-32$.

Skinner, E. A., \& Pitzer, J. R. (2012). Developmental dynamics of student engagement, coping and everyday resilience. In A. Christenson, A. Reschly \& C. Wylie(Eds.), Handbook of research on student engagement (pp. 21-44). New York: Springer 
Skinner, E. A., Zimmer-Gembeck, M. J., Connell, J. P., Eccles, J. S., \& Wellborn, J. G. (1998). Individual differences and the development of perceived control. Monographs of the Society for Research in Child Development, 63(2/3).

Steele, C. M. (1997). A threat in the air: How stereotypes shape intellectual identity and performance. American Psychologist, 52(6), 613 - 629.

Tucker, C. M., Zayco, R. a., Herman, K. C., Reinke, W. M., Trujillo, M., Carraway, K., Ivery, P. D. (2002). Teacher and child variables as predictors of academic engagement among low-income African American children. Psychology in the Schools, 39(4), 477-488.

Van Dyke, C. J., \& Elias, M. J. (2007). How forgiveness, purpose, and religiosity are related to the mental health and well-being of youth: A review of the literature. Mental Health, Religion \& Culture, 10(4), 395-415.

Van Ryzin, M. J., Gravely, A. a, \& Roseth, C. J. (2009). Autonomy, belongingness, and engagement in school as contributors to adolescent psychological well-being. Journal of Youth and Adolescence, 38(1), 1-12.

Van Ryzin, M. J. (2011). Protective factors at school reciprocal effects among adolescents' perceptions of the school environment, engagement in learning, and hope. Journal of Youth and Adolescence, 1568-1580. 
Walton, G. M., \& Cohen, G. L. (2011). A brief social-belonging intervention improves academic and health outcomes of minority students. Science, 331(March), 14471451.

Wellborn, J. G., \& Connell, J. P. (1991). Engagement versus disaffection: Motivated patterns of action in the academic domain (Unpublished Dissertation). Rochester, NY: University of Rochester.

Wentzel, K. (2009). Peers and academic functioning at school. In B. Rubin, Kenneth H., Bukoski, William M., Laursen (Ed.), Handbook of peer interactions, relationships, and groups: Social, emotional, and personality development in context (pp. 531547). New York: Guilford Press.

Wentzel, K. R., \& Caldwell, K. (1997). Friendships, peer acceptance, and group membership: relations to academic achievement in middle school. Child Development, 68(6), 1198-209.

Wentzel, K. R., Battle, A., Russell, S. L., \& Looney, L. B. (2010). Social supports from teachers and peers as predictors of academic and social motivation. Contemporary Educational Psychology, 35(3), 193-202.

Wigfield, A., Eccles, J. S., Roeser, R. W., \& Schiefele. (2008). Development of achievement motivation. In W. Damon \& R. M. Lerner (Eds.), Child and 
References

adolescent development: An advanced course (Vol. 4, pp. 406-425). Hoboken, NJ: Wiley \& Sons.

Wigfield, A., Eccles, J. S., Schiefele, U., Roeser, R. W., \& Davis-Kean, P. (2006). Development of achievement motivation. In N. Eisenberg, W. Damon, \& R. M. Lerner (Eds.), Handbook of child psychology, volume three: Social, emotional, and personality development (6th ed., pp. 933-1002). Hoboken, NJ: Wiley \& Sons.

Wigfield, A., Eccles, J., \& Yoon, K. (1997). Change in children's competence beliefs and subjective task values across the elementary school years: A 3-year study. Journal of Educational Psychology, 89(3), 451-569.

Williams, D. R., \& Brown J. D. (2011). Learning gardens and sustainability education: Bringing life to schools and schools to life. New York, NY: Routledge.

Yates, T. M., Egeland, B., \& Sroufe, A. (2003). Rethinking resilience. In S. Luthar (Ed.), Resilience and vulnerability: Adaptation in the context of childhood adversities (pp.243-266). New York: Cambridge University Press.

Yeager, D. S., \& Bundick, M. J. (2009). The role of purposeful work goals in promoting meaning in life and in schoolwork during adolescence. Journal of Adolescent Research, 24(4), 423-452.

Zimmer-Gembeck, M. J., Chipuer, H. M., Hanisch, M., Creed, P. A, \& McGregor, L. (2006). Relationships at school and stage-environment fit as resources for adolescent engagement and achievement. Journal of Adolescence, 29(6), 911-933. 


\section{APPENDIX A}

\section{MEASURES}

\section{Engagement Profile (11 items)}

\section{Behavioral Engagement}

- I try hard to do well in school. $(+)$

Behavioral Disaffection

- When I'm in class, I just act like I'm working. (-)

- In school, I don't work very hard. (-)

Emotional Engagement

- I look forward to coming to school $(+)$

- I enjoy learning new things in school. $(+)$

Emotional Disaffection

- When I'm in class, I feel mad. (-)

- I can't stand doing school work. (-)

- When we work on something in class, I feel bored. (-)

- School makes me angry. (-)

\section{Amotivation}

- I really don't care about getting good grades. (-)

- I really don't care about school. (-)

\section{Autonomy (4 items)}

\section{Academic Identified Self-Regulation}

- Because I want to understand the subject. $(+)$

- Because homework helps me learn more. $(+)$

- Because we are learning important things. $(+)$

- Because doing well in school is important to me. $(+)$ 


\section{Teacher Motivational Support (9 items)}

\section{Structure}

- My teacher treats me fairly. $(+)$

- I know what my teacher expects of me in class. $(+)$

- My teachers keep changing the rules in our class. (-)

Autonomy Support:

- My teachers explain why the things I learn in school are important. $(+)$

- My teachers don't give me much choice about how I do my schoolwork. (-)

- My teachers never talk about how I can use the things we learn in school. (-) Involvement

- My teachers just don't understand me. (-)

- I can't really count on my teachers. (-)

- Sometimes I wonder if my teachers really like me. (-)

\section{Peer Relationships (12 items)}

Peer Attachment

- I can really be myself with my friends. $(+)$

- I feel close to my friends. (+)

- When I'm with my friends, I feel bad. (-)

- I feel like an outsider with my friends. (-)

- I feel that my friends don't understand me. (-)

Peer Support

- My friends understand me. $(+)$

- My friends respect and appreciate me. $(+)$

- My friends are always ordering me around. (-)

- Sometimes I wonder if my friends like me. (-)

Peer Engagement

- My friends and I enjoy hanging out together. $(+)$

- My friends and I care about each other. $(+)$

- Right now I feel stuck with my friends. (-) 


\section{Garden-based Education Engagement (13 items)}

Behavioral Engagement

- I try hard to do well in the garden. $(+)$

- When I'm in the garden, I work as hard as I can. $(+)$

- When I'm in the garden, I listen carefully to our gardening teacher. $(+)$

Behavioral Disaffection

- When I'm in the garden, I just act like I'm working. (-)

- When I'm in the garden, I can't wait for it to be over. (-)

- In the garden, I have noticed that my mind wanders, and I think about other things. (-)

Emotional Engagement

- I am surprised that I like it. (+)

- When I'm in the garden, I feel good. $(+)$

- Gardening is interesting. $(+)$

- Working in the garden is fun. $(+)$

Emotional Disaffection

- When I'm in the garden, I am wet, dirty, not really interested in what we are doing. (-)

- I'd rather be doing just about anything else but gardening. (-)

- Gardening is boring. (-)

Amotivation

- I'd rather buy food at the grocery store. (-)

- I don't care about gardening. (-)

- I don't care if I miss gardening class. (-) 
APPENDIX B

SUPPLEMENTARY INFORMATION: ANOVAS 


\section{Research Question 2a}

Table A1

Eighth-grade Mean Levels and Pairwise Differences: Motivational Resilience Group.

\begin{tabular}{|c|c|c|c|c|c|c|c|c|c|}
\hline \multirow[t]{4}{*}{$\begin{array}{l}\text { Developmental } \\
\text { Resource }\end{array}$} & \multicolumn{6}{|c|}{ Mean Levels } & \multicolumn{3}{|c|}{$\begin{array}{l}\text { Mean Differences for Significant Pairwise } \\
\text { Comparisons }\end{array}$} \\
\hline & \multicolumn{6}{|c|}{ Motivational Resilience Group } & \multicolumn{3}{|c|}{ Motivational Resilience Group } \\
\hline & \multicolumn{2}{|c|}{$\begin{array}{c}\text { Stress- } \\
\text { Affected } \\
\text { (Str.) } n=84\end{array}$} & \multicolumn{2}{|c|}{$\begin{array}{c}\text { Average } \\
(A v .) n=55\end{array}$} & \multicolumn{2}{|c|}{$\begin{array}{c}\text { Resilient } \\
\text { (Res.) } n=28\end{array}$} & Av. - Str. & Res. - Str. & Res. - Av. \\
\hline & & & Mean & (SD) & Mean & (SD) & M. diff. & M. diff. & M. diff. \\
\hline Autonomy & 3.18 & $(.87)$ & 3.78 & $(.78)$ & 4.62 & $(.49)$ & $.60^{* * *}$ & $1.44^{* * *}$ & $.83^{* * *}$ \\
\hline Teacher Support & 3.78 & $(.56)$ & 3.98 & $(.40)$ & 4.46 & $(.42)$ & $.20^{\dagger}$ & $.68^{* * *}$ & $.48^{* * *}$ \\
\hline Peer Support & 4.14 & $(.56)$ & 4.32 & $(.43)$ & 4.47 & $(.44)$ & -- & $.32^{* *}$ & -- \\
\hline \multirow[t]{4}{*}{ Garden Engagement } & 3.26 & $(.80)$ & 3.31 & $(.61)$ & 3.69 & $(.85)$ & -- & $.42^{*}$ & $.37^{\dagger}$ \\
\hline & \multicolumn{6}{|c|}{ Gender } & \multicolumn{3}{|c|}{ Gender } \\
\hline & \multicolumn{2}{|c|}{$\begin{array}{c}\text { Male } \\
n=88\end{array}$} & \multicolumn{2}{|c|}{$\begin{array}{c}\text { Female } \\
n=79\end{array}$} & & & Female - Male & & \\
\hline & Mean & (SD) & Mean & (SD) & & & $M$. diff. & & \\
\hline Autonomy & 3.44 & $(1.01)$ & 3.82 & $(.83)$ & & & $.39^{* *}$ & & \\
\hline Teacher Support & 3.90 & $(.61)$ & 4.03 & $(.45)$ & & & $.14^{\dagger}$ & & \\
\hline Peer Support & 4.14 & $(.58)$ & 4.38 & $(.38)$ & & & $.24^{* *}$ & & \\
\hline Garden Engagement & 3.31 & $(.70)$ & 3.78 & (.83) & & & -- & & \\
\hline
\end{tabular}




\section{Research Question 2b}

Table A2

Sixth-grade Mean Levels and Pairwise Differences: Motivational Resilience Group.

\begin{tabular}{|c|c|c|c|c|c|c|c|c|c|}
\hline \multirow[t]{4}{*}{$\begin{array}{l}\text { Developmental } \\
\text { Resource }\end{array}$} & \multicolumn{6}{|c|}{ Mean Levels } & \multirow{2}{*}{\multicolumn{3}{|c|}{$\begin{array}{c}\text { Mean Differences for Significant } \\
\text { Pairwise Comparisons } \\
\text { Motivational Resilience Group }\end{array}$}} \\
\hline & \multicolumn{6}{|c|}{ Motivational Resilience Group } & & & \\
\hline & \multicolumn{2}{|c|}{$\begin{array}{c}\text { Stress-Affected } \\
\text { (Str.) } n=135\end{array}$} & \multicolumn{2}{|c|}{$\begin{array}{c}\text { Average } \\
(A v .) n=108\end{array}$} & \multicolumn{2}{|c|}{$\begin{array}{c}\text { Resilient } \\
\text { (Res.) } n=79\end{array}$} & \multirow{2}{*}{$\begin{array}{c}\text { Av. }- \text { Str. } \\
M \text { diff. }\end{array}$} & \multirow{2}{*}{$\frac{\text { Res. }- \text { Str. }}{M \text { diff. }}$} & \multirow{2}{*}{$\frac{\text { Res. }- \text { Av. }}{M \text { diff. }}$} \\
\hline & Mean & (SD) & Mean & (SD) & Mean & (SD) & & & \\
\hline Autonomy & 3.18 & $(1.01)$ & 3.98 & $(.74)$ & 4.51 & $(.57)$ & $.79^{* * *}$ & $1.33^{* * *}$ & $.53^{* *}$ \\
\hline Teacher Support & 3.66 & $(.61)$ & 4.10 & $(.47)$ & 4.41 & $(.42)$ & $.43^{* * *}$ & $.75^{* * *}$ & $.32^{* *}$ \\
\hline Peer Support & 4.10 & $(.59)$ & 4.27 & $(.49)$ & 4.32 & $(.64)$ & -- & -- & -- \\
\hline $\begin{array}{l}\text { Garden } \\
\text { Engagement }\end{array}$ & 3.57 & $(.76)$ & 3.80 & $(.62)$ & 4.17 & $(.60)$ & -- & $.59^{* * *}$ & -- \\
\hline
\end{tabular}




\section{Research Question 2c}

\section{Table A3}

Combined Grades ANCOVAs and Mean Levels: Motivational Resilience Groups.

Developmental Resource

Mean Levels

\begin{tabular}{|c|c|c|c|c|c|c|}
\hline & & \multicolumn{5}{|c|}{ Motivational Resilience Group } \\
\hline & & \multirow{2}{*}{$\begin{array}{c}\text { Stress-Affected } \\
\text { (Str.) } n=135 \\
\text { Mean (SD) }\end{array}$} & \multicolumn{2}{|c|}{$\begin{array}{c}\text { Average } \\
(A v .) n=108\end{array}$} & \multicolumn{2}{|c|}{$\begin{array}{c}\text { Resilient } \\
\text { (Res.) } n=7\end{array}$} \\
\hline & & & Mean & $(\mathrm{SD})$ & Mean & $(\mathrm{SD}$ \\
\hline Autonomy & 3.18 & $(.92)$ & 3.88 & $(.77)$ & 4.55 & \\
\hline \multicolumn{7}{|c|}{$F(2,315)=70.82, p<.001$, partial $\eta^{2}=.31}$. \\
\hline Teacher Support & 3.74 & $(.58)$ & 4.04 & $(.44)$ & 4.43 & \\
\hline \multicolumn{7}{|c|}{$F(2,315)=44.16, p<.001$, partial $\eta^{2}=.23$} \\
\hline Peer Support & 4.13 & $(.59)$ & 4.30 & $(.46)$ & 4.37 & \\
\hline \multicolumn{7}{|c|}{$F(2,315)=4.57, p=.01$, partial $\eta^{2}=.03$. } \\
\hline Garden Engageme & 3.38 & $(.80)$ & 3.55 & $(.66)$ & 4.00 & 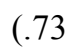 \\
\hline
\end{tabular}

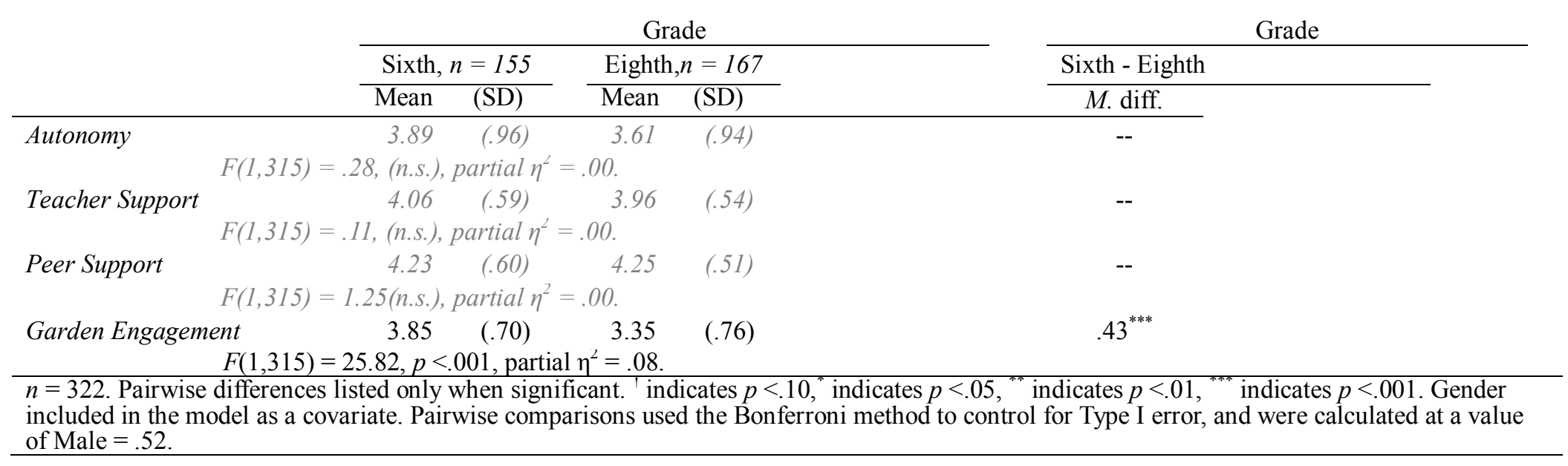

Mean Differences for Significant Pairwise \begin{tabular}{c} 
Comparisons \\
\hline Motivational Resilience Group
\end{tabular}

\begin{tabular}{ccc}
\hline Av. - Str. & Res. - Str. & Res. - Av. \\
\hline M. diff. & $M$. diff. & $M$. diff. \\
\hline $.70^{* * *}$ & $1.35^{* * *}$ & $.66^{* * *}$
\end{tabular}

$.31^{* * *}$

$.71^{* * *}$

$.40^{* * *}$

$.16^{\dagger}$

$.24^{* *}$

$--$

$-{ }^{-} \quad .51^{* *}$

$.37^{* *}$

\section{逽

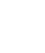




\section{Research Question 3a}

Table A4

Eighth-grade Mean Levels and Pairwise Difference for Academic Resilience Group.

Developmental

Resource

Mean Levels

\begin{tabular}{|c|c|c|c|c|c|c|}
\hline & \multicolumn{6}{|c|}{ Academic Resilience Group } \\
\hline & \multicolumn{2}{|c|}{$\begin{array}{c}\text { Stress-Affected } \\
\text { (Str.) } n=90\end{array}$} & \multicolumn{2}{|c|}{$\begin{array}{c}\text { Average } \\
(A v .) n=41\end{array}$} & \multicolumn{2}{|c|}{$\begin{array}{c}\text { Resilient } \\
\text { (Res.) } n=36\end{array}$} \\
\hline & Mean & (SD) & Mean & (SD) & Mean & (SD) \\
\hline Autonomy & 3.53 & (96) & 3.50 & $(.95)$ & 3.99 & $(.83)$ \\
\hline Teacher Support & 3.88 & $(.55)$ & 3.98 & $(.56)$ & 4.17 & $(.47)$ \\
\hline Peer Support & 4.21 & $(.57)$ & 4.31 & $(.45)$ & 4.32 & $(.42)$ \\
\hline $\begin{array}{l}\text { Garden } \\
\text { Engagement }\end{array}$ & 3.23 & (.69) & 3.37 & $(.84)$ & 3.61 & $(.79)$ \\
\hline
\end{tabular}

Engagement
Mean Differences for Significant Pairwise Comparisons

Academic Resilience Group

Av. - Str. $\quad$ Res. - Str. $\quad$ Res. - Av.

$M$ diff. $\quad M$ diff. $\quad M$ diff.

$\begin{array}{llll}-- & .46^{*} & .49^{\dagger}\end{array}$

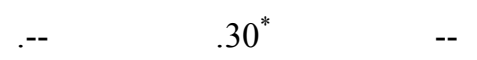

$n=167$. Pairwise differences listed only when significant. ${ }^{\dagger}$ indicates $p<.10,{ }^{*}$ indicates $p<.05,{ }^{* *}$ indicates $p<.01$

indicates $p<.001$. Gender included in the model as a covariate. Pairwise comparisons used the Bonferroni method to control for Type I error, and were calculated at a value of gender $=.53$ 


\section{Research Question 3c.}

\section{Table A4}

Combined Grades ANOVAs and Mean Levels: Academic Resilience Groups

Developmental

Mean Levels

Resource

Adjusted Mean Differences for

Significant Pairwise Comparisons

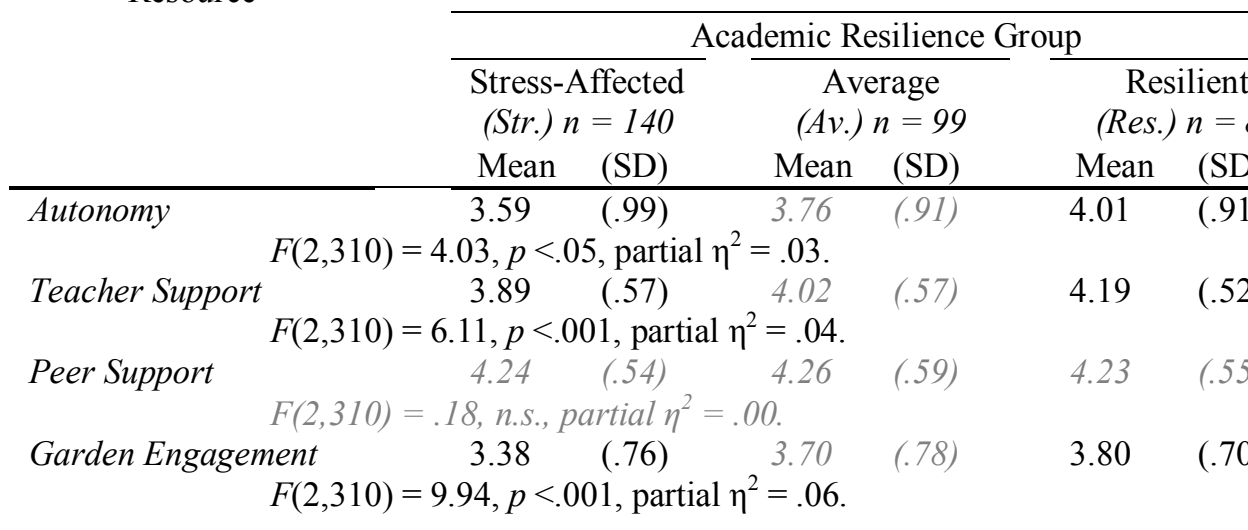

Academic Resilience Group

Grade

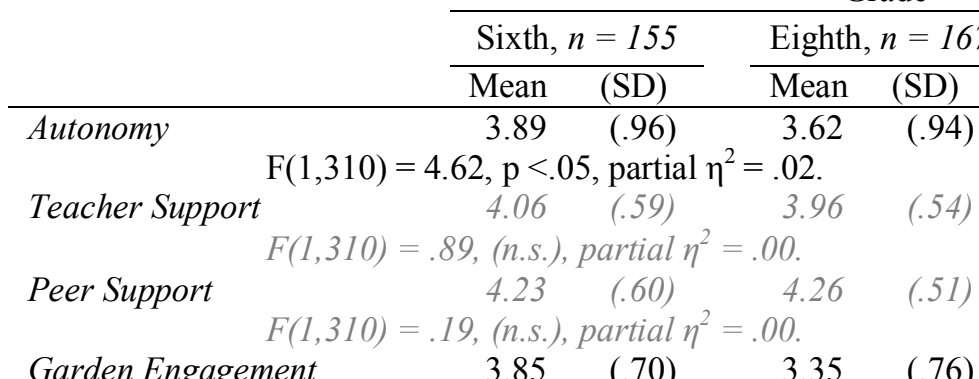

Garden Engagement $\quad 3.85 \quad(.70)$

$$
F(1,310)=28.15, p<.001, \text { partial } \eta^{2}=.08 \text {. }
$$

$n=322$. Grey italic cells indicate values for which there are no significant pairwise comparisons. Pairwise differences listed only when significant. indicates $p<.10,{ }^{*}$ indicates $p<.05,{ }^{* *}$ indicates $p<.01,{ }^{* * *}$ indicates $p<.001$. Gender included in the model as a covariate. Pairwise comparisons used the Bonferroni method to control for Type I error, and were calculated at a value of Male $=.52$. 


\section{Research Question 4a}

Table A5

Eighth-grade Mean Levels and Pairwise Differences: Dual Resilience Group

Developmental Resource

Stress-Affected
(Str.) $n=84$
Mean (SD)

\begin{tabular}{|c|c|c|c|c|c|c|}
\hline & \multicolumn{2}{|c|}{ (Str.) $n=84$} & \multicolumn{2}{|c|}{$(A v) n=55}$. & \multicolumn{2}{|c|}{ (Res.) $n=28$} \\
\hline & Mean & & Mean & $(\mathrm{SD})$ & Mean & (SD) \\
\hline Autonomy & 3.20 & $(93)$ & 3.72 & $(.88)$ & 4.50 & $(.56)$ \\
\hline Teacher Support & 3.76 & $(.59)$ & 4.01 & $(.49)$ & 4.11 & $(.46)$ \\
\hline Peer Support & 4.15 & $(.63)$ & 4.30 & $(.45)$ & 4.39 & $(.44)$ \\
\hline Garden Engagement & 3.18 & $(.81)$ & 3.37 & $(.68)$ & 3.95 & $(.88)$ \\
\hline
\end{tabular}

\begin{tabular}{|c|c|c|c|c|c|c|}
\hline & \multicolumn{4}{|c|}{ Gender } & \multicolumn{2}{|r|}{ Grade } \\
\hline & \multicolumn{2}{|c|}{$\begin{array}{c}\text { Male } \\
n=88\end{array}$} & \multicolumn{2}{|c|}{$\begin{array}{c}\text { Female } \\
n=79\end{array}$} & \multirow{2}{*}{$\begin{array}{c}\text { Male - Female } \\
M . \text { diff. }\end{array}$} & \\
\hline & Mean & (SD) & Mean & (SD) & & \\
\hline Autonomy & 3.44 & $(1.01)$ & 3.82 & $(.83)$ & $.39^{* *}$ & \\
\hline Teacher Support & 3.90 & $(.61)$ & 4.03 & $(.45)$ & $.14^{\dagger}$ & \\
\hline Peer Support & 4.14 & $(.58)$ & 4.38 & $(.38)$ & $24^{* *}$ & \\
\hline Garden Engagement & 3.31 & $(.70)$ & 3.78 & $(.83)$ &.-- & \\
\hline
\end{tabular}

Adjusted Mean Differences for Significant Pairwise Comparisons Dual Resilience Group

\begin{tabular}{ccc}
\multicolumn{3}{c}{ Dual Resilience Group } \\
\hline Av. - Str. & Res. - Str. & Res. - Av. \\
\hline M. diff. & M. diff. & M. diff. \\
\hline $.48^{* *}$ & $1.13^{* * *}$ & $.66^{\dagger}$ \\
$.23^{*}$ & $.66^{* *}$ & $.43^{\dagger}$ \\
-- & -- & -- \\
-- & $.33^{* *}$ & --
\end{tabular}

Resilience Group

$\begin{array}{ccc}\text { Average } & & \text { Resilient } \\ (A v .) n=55 & & \text { (Res.) } n=28 \\ \text { Mean } \quad \text { (SD) } & & \text { Mean } \quad \text { (SD) }\end{array}$

$M$.

23 


\section{Research Question 4b}

Table A6

Sixth-grade Mean Levels and Pairwise Differences: Dual Resilience Group.

\begin{tabular}{|c|c|c|c|c|c|c|c|c|c|}
\hline \multirow[t]{4}{*}{$\begin{array}{l}\text { Developmental } \\
\text { Resource }\end{array}$} & \multicolumn{6}{|c|}{ Mean Levels } & \multicolumn{3}{|c|}{$\begin{array}{c}\text { Mean Differences for Significant } \\
\text { Pairwise Comparisons }\end{array}$} \\
\hline & \multicolumn{6}{|c|}{ Motivational Resilience Group } & \multicolumn{3}{|c|}{ Motivational Resilience Group } \\
\hline & \multicolumn{2}{|c|}{$\begin{array}{l}\text { Stress-Affected } \\
\text { (Str.) } n=135\end{array}$} & \multicolumn{2}{|c|}{$\begin{array}{c}\text { Average } \\
(A v .) n=108\end{array}$} & \multicolumn{2}{|c|}{$\begin{array}{c}\text { Resilient } \\
\text { (Res.) } n=79\end{array}$} & Av. - Str. & Res. - Str. & Res. - Av. \\
\hline & Mean & $(\mathrm{SD})$ & Mean & (SD) & Mean & $(\mathrm{SD})$ & $M$ diff. & $M$ diff. & $M$ diff. \\
\hline Autonomy & 3.16 & $(1.06)$ & 3.89 & $(.88)$ & 4.68 & $(.52)$ & $.74^{* *}$ & $1.42^{* * *}$ & $.79^{* *}$ \\
\hline Teacher Support & 3.66 & $(.65)$ & 4.06 & $(.55)$ & 4.48 & $(.39)$ & $.40^{* *}$ & $.82^{* * *}$ & $.42^{* *}$ \\
\hline Peer Support & 4.20 & $(.51)$ & 4.23 & $(.62)$ & 4.26 & $(.60)$ & -- & -- & -- \\
\hline $\begin{array}{l}\text { Garden } \\
\text { Engagement }\end{array}$ & 3.53 & $(.88)$ & 3.81 & $(.65)$ & 4.36 & $(.43)$ & -- & $.83^{* * *}$ & $.55^{* *}$ \\
\hline
\end{tabular}

$n=155{ }^{\dagger}$ indicates $p<.10,{ }^{*}$ indicates $p<.05,{ }^{* *}$ indicates $p<.01,{ }^{* * *}$ indicates $p<.001$. Pairwise comparisons used the Bonferroni method to control for Type I error, and are listed only when significant. 


\section{Research Question 4c}

Table A7

Combined Grades ANOVAs and Mean Levels: Gender and Grade Developmental Resource

Mean Levels

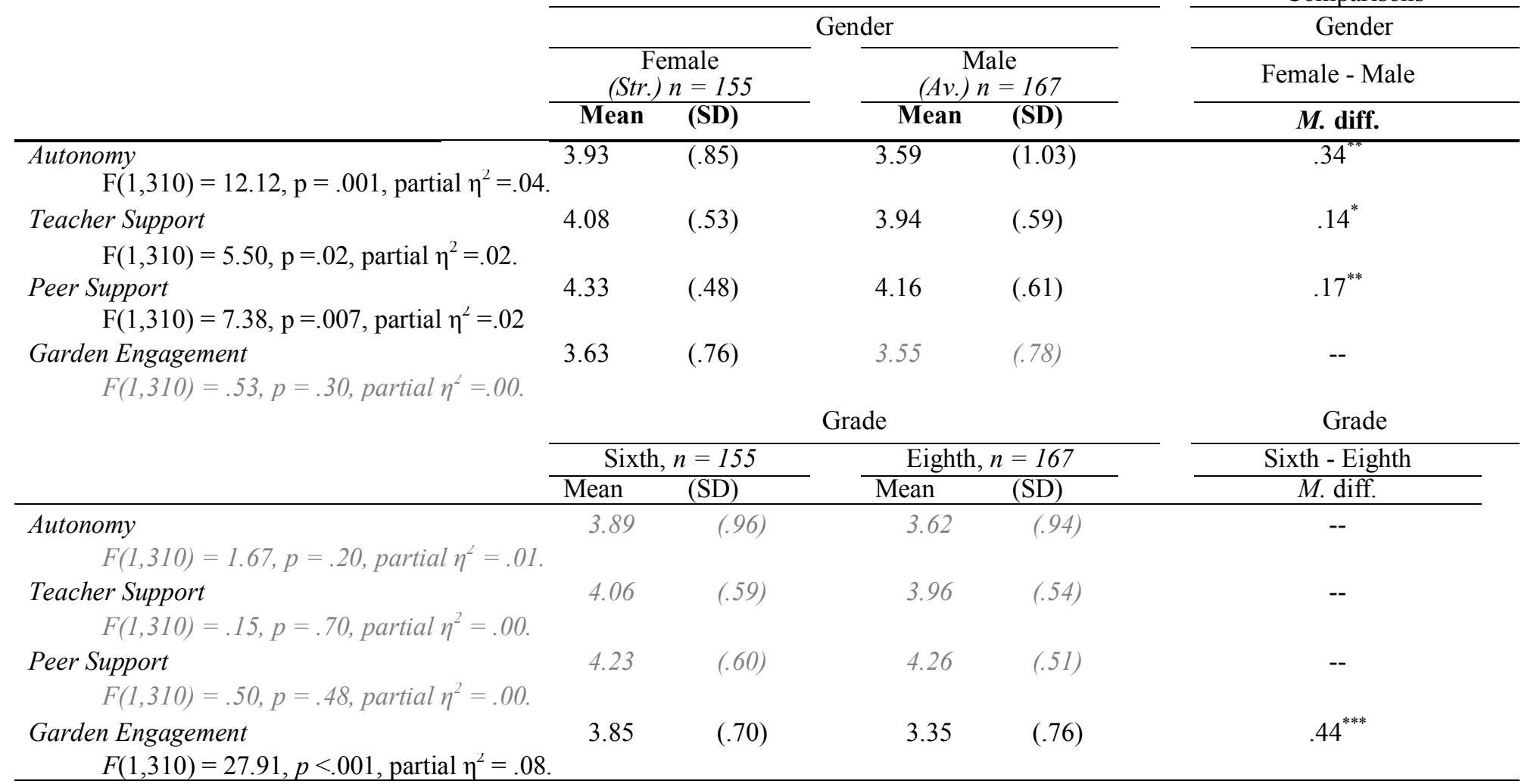

Gender Comparisons

Mean Differences for

$n=322$. Grey italic cells indicate values for which there are no significant pairwise comparisons. Pairwise differences listed only when significant. ${ }^{\top}$ indicates $p<.10,{ }^{*}$ indicates $p<.05,{ }^{* *}$ indicates $p<.01,{ }^{* * *}$ indicates $p<.001$. 
Appendix

\section{APPENDIX C \\ SUPPLEMENTARY INFORMATION: UNIQUE EFFECTS}

Research Question 5a3:

\section{Further Interpretation of Multigroup Model Invariance Testing}

The invariance testing process corroborated the findings of sub-question c from research questions 2 - 4, which tested for but did not find significant grade by resilience group interaction terms. Patterns of prediction did vary between groups, but the overarching message from the results seemed to be that the developmental resources are collectively important for both grades. In the final model tested here, where regression paths were held to be equal across grades and only one correlation was allowed to vary, each of the developmental resources and gender was a significant unique predictor of dually resilient group membership (although the effect of peer support, again, showed evidence of being influenced by suppression.) Autonomy and teacher support were significant predictors of dually stress-affected group membership as well as dually resilient group membership. This, again, corroborates the findings from research questions 2-4 that each of the developmental resources seemed to be important for motivational or academic success, with peer support as the weakest resource, and autonomy and teacher support as the strongest resources. Considered in conjunction with the regression analyses, the findings of significant effects for each of the resources in this combined-grades model could suggest that, with a larger sample size and correspondingly higher power, all of the resources might emerge as significant predictors for both grades. Considering the relatively low $p$-values of the 
non-significant teacher, garden, and gender effects in the eighth-grade model (around $p=.15$ ), it is important to note that the focus of research question five is not on whether the resources are general resources for overall motivation and academic success, which they seem to be, but rather on whether they serve as enabling (encouraging being better than average) or protective (discouraging being worse than average) resources.

The descriptive differences in the pattern of unique connections found between the sixth and eighth graders' regression paths might be better understood in light of the differences in relationships among the developmental resources that emerged from invariance testing. The apparent primary source of differences between the two grades was the relationship between autonomy and teacher support. This correlation was present for both grades in this partially constrained model, but was stronger for sixth graders $\left(r=.47^{* * *}\right)$ than for eighth graders $\left(r=.27^{* * *}\right)$. The cause for this stronger correlation is unknown, but suggests that, for eighth graders, levels of autonomy might drive motivational and academic success with less reference to levels of teacher support, whereas for sixth graders, these resources might function less discriminately and more cohesively.

The other correlation that appeared to differ between grades was that between autonomy and garden engagement. When allowed to vary, this association was relatively strong for sixth graders $\left(r=.34^{* * *}\right)$ and not significant for eighth graders. Again, the cause of this difference is unknown, but it could be that garden engagement simply function as one positive aspect in a relatively homogenous school experience 
for sixth graders, but, for eighth graders, is a (weak) resource for motivation that does not depend on students' sense of autonomy over schoolwork.

Figure A1. Multi-group Multinomial Logistic SEM: Unique Effects of Developmental Resources on Dual Resilience Group Membership, Final PartiallyConstrained Model.

Odds Ratios and Correlations: Both Grades, partially-constrained model, $n=322$. (Correlation between autonomy and teacher support allowed to vary between grades; $6^{\text {th }}$ grade correlation in parentheses.)

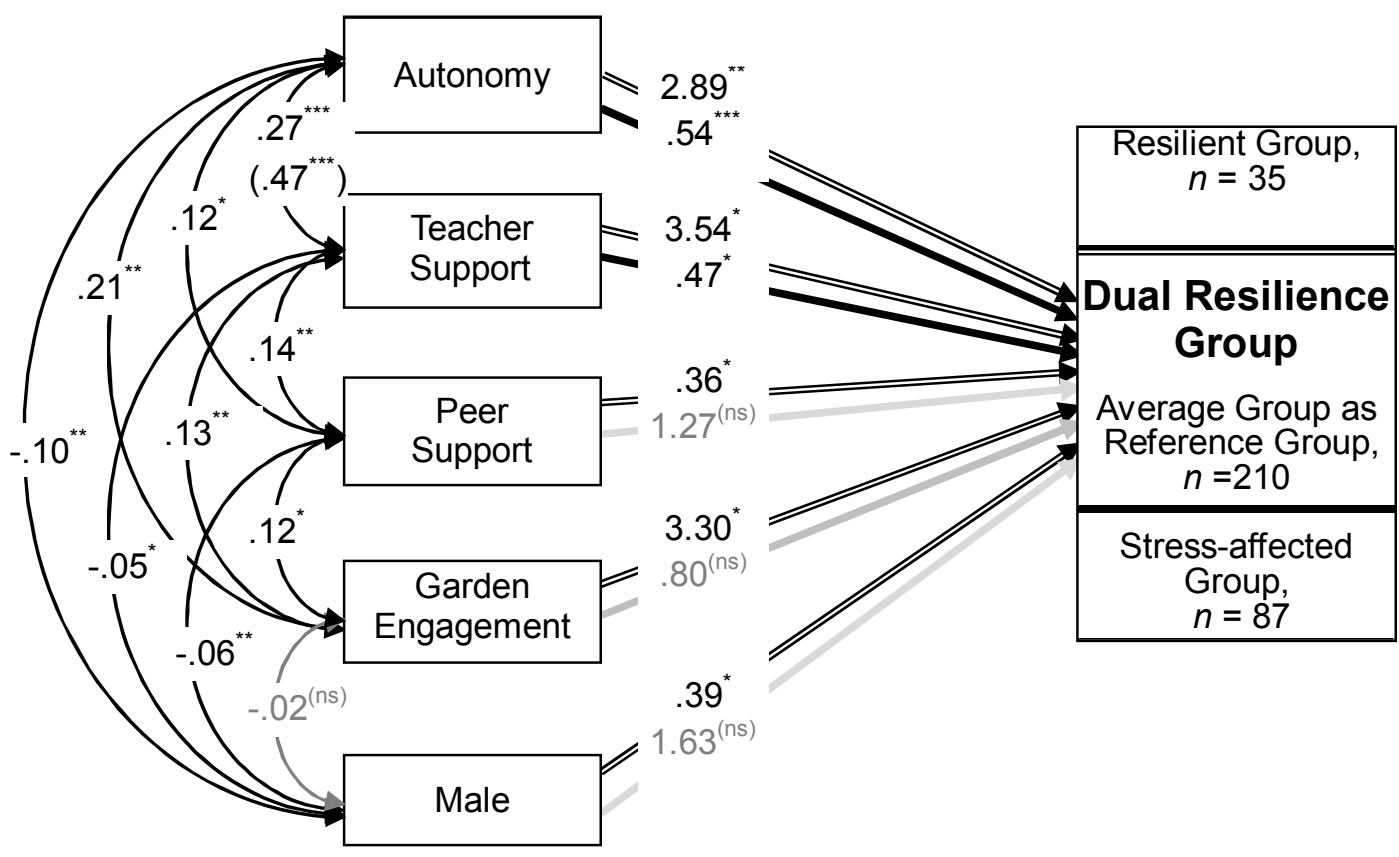

Model information:

Loglikelihood $=-1708.90(41)$, Scaling factor $1.10 ; X^{2}(10)=91.03, p<.001 ;$ AIC $=3499.79, \mathrm{BIC}=3654.55$. Note: $n=322$. ${ }^{* * *} p<.001,{ }^{* *} p<.01,{ }^{*} p<.05,{ }^{\dagger} p<.10,{ }^{(\mathrm{ns})} p<.10$. Grey coloring indicates nonsignificant path or correlation. Hollow ( $($ ) lines indicate paths predicting dually resilient (vs. average) group membership; solid $($ ) lines indicate paths predicting dually stress-affected (vs. average) group membership. Values on paths are the odds of being in the selected group (vs. the average group) that correspond to each unit increase in the IV, where $>1$ is increased likelihood, 1 is equal likelihood, and $<1$ is decreased likelihood. Gender coded such that female $=0$ and male $=1$. All developmental resources 


\section{APPENDIX D}

\section{SUPPLEMENTARY ANALYSES: MEDIATIONS}

\section{Preliminary Regressions Predicting Dual Resilience Group Membership}

In the preliminary analyses for this research question, multinomial logistic regression models were created for each developmental resource to test whether these resources predicted dual resilience group for eighth graders, controlling for gender. Separate models tested eighth-grade autonomy, teacher support, peer support, and garden engagement, respectively. In each model, the two IVs were the respective developmental resource and gender, dual resilience group membership was the nominal DV with two predicted outcomes: dually resilient (vs. average) or dually stress-affected (vs. average) group membership dual resilience group. The results from the eighth-grade models were then compared with those from the sixth-grade models to identify any potential differences among the developmental resources in their prediction of dual resilience group.

\section{Eighth graders: when tested separately, do each of the four developmental resources predict dual resilience group for eighth graders?}

For eighth-grade students, autonomy, teacher support, and garden engagement each predicted dual resilience group membership, when controlling for gender. Peer support did not predict dual resilience group when controlling for gender. Regression slopes, $p$-values, standard errors, $z$-scores, odds ratios, and confidence intervals are reported along with model fit information for each model in Table A9 .

When controlling for gender, autonomy seemed to serve both as an enabling 
resource for eighth-grade students' dual resilience and a protective resource against their stress-affectedness. The combination of autonomy and gender significantly explained dual resilience group membership, as compared with the intercepts only model, $X^{2}(4)=28.26, p<.001$. When controlling for gender, a one unit increase in autonomy corresponded to being 2.35 times as likely for students to be members of the resilient group as it was for them to be members of the average group, $p=.02$, and .45 times (about half) as likely for students to be members of the stress-affected group as it was for them to be members of the average group, $p=.002$. After controlling for autonomy, gender was not a significant predictor of resilience group.

When controlling for gender, teacher support also seemed to serve as both an enabling resource for eighth-grade students' dual resilience and a protective resource against students' dual stress-affectedness. The combination of teacher support and gender significantly explained dual resilience group membership, as compared with the intercepts only model, support $X^{2}(4)=20.80, p<.001$. When controlling for gender, a one unit increase in teacher support corresponded to it being 3.45 times as likely for students to be members of the resilient group as it was for them to be members of the average group, $p=.04$, and .34 times (about one third) as likely for students to be members of the stress-affected group as it was for them to be members of the average group, $p=.004$. After controlling for teacher support, gender was a marginally significant predictor of resilience group, such that being male instead of female corresponded to being .28 times (about one quarter) as likely for students to be 
Table A8

Developmental Resources as Predictors of Eighth-grade Dual Resilience Group: Multinomial Logistic Regressions.

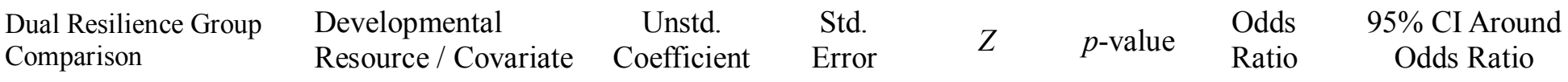

\begin{tabular}{|c|c|c|c|c|c|c|c|}
\hline \multicolumn{8}{|c|}{ Autonomy, controlling for gender: Loglikelihood $=-412.06(11)$, Scaling factor $=.85$, AIC $=846.12$, BIC $=880.42$. } \\
\hline \multirow{2}{*}{$\begin{array}{l}\begin{array}{l}\text { Stress-affected } \\
\text { (vs. average) }\end{array} \\
\end{array}$} & Autonomy & -.80 & .23 & -3.53 & .000 & .45 & $.31-.65$ \\
\hline & Male & .15 & .41 & .36 & .72 & 1.16 & $.59-2.30$ \\
\hline \multirow{2}{*}{$\begin{array}{l}\text { Resilient } \\
\text { (vs.average) }\end{array}$} & Autonomy & .86 & .36 & 2.40 & .02 & 2.35 & $1.31-4.23$ \\
\hline & Male & -1.08 & .72 & -1.50 & .13 & .34 & $.11-1.11$ \\
\hline \multicolumn{8}{|c|}{ Teacher Support, controlling for gender: Loglikelihood $=-358.91$ (11), Scaling factor $=.93$, AIC $=739.81$, BIC $=774.11$} \\
\hline \multirow{2}{*}{$\begin{array}{l}\text { Stress-affected } \\
\text { (vs. average) }\end{array}$} & Teacher Support & -1.07 & .37 & -2.88 & .004 & .34 & $.19-.63$ \\
\hline & Male & .35 & .37 & .93 & .35 & 1.41 & $.77-2.62$ \\
\hline \multirow{2}{*}{$\begin{array}{l}\text { Resilient } \\
\text { (vs.average) }\end{array}$} & Teacher Support & 1.24 & .59 & 2.08 & .04 & 3.45 & $1.30-9.15$ \\
\hline & Male & -1.27 & .71 & -1.79 & .07 & .28 & $.09-.90$ \\
\hline
\end{tabular}

Peer Support, controlling for gender: Loglikelihood $=-354.11$ (11), Scaling factor $=1.19$, AIC $=730.22$, BIC $=764.51$.

(Overall model not significantly different than intercepts-only model.)

\begin{tabular}{llcccccc}
\hline $\begin{array}{l}\text { Stress-affected } \\
\text { (vs. average) }\end{array}$ & Peer Support & -.43 & .39 & -1.12 & .26 & .65 & $.34-1.23$ \\
\hline Resilient & Male & .29 & .38 & .76 & .45 & 1.33 & $.72-2.47$ \\
(vs.average) & Peer Support & -.37 & .43 & -.86 & .39 & .69 & $.34-1.40$ \\
\hline & Male & -1.39 & .76 & -1.84 & .07 & .25 & $.07-.86$
\end{tabular}

Garden Engagement, controlling for gender: $\quad$ Loglikelihood $=-420.84$ (11), Scaling factor $=.99$, AIC $=863.67$, BIC $=897.97$.

\begin{tabular}{|c|c|c|c|c|c|c|c|}
\hline $\begin{array}{l}\text { Stress-affected } \\
\text { (vs. average) }\end{array}$ & $\begin{array}{l}\text { Garden Engagement } \\
\text { Male }\end{array}$ & $\begin{array}{l}-.36 \\
.45\end{array}$ & .24 & $\begin{array}{l}-1.49 \\
1.27\end{array}$ & $\begin{array}{l}.14 \\
.20\end{array}$ & $\begin{array}{l}.70 \\
1.57\end{array}$ & $\begin{array}{l}.47-1.04 \\
.88-2.81\end{array}$ \\
\hline $\begin{array}{l}\text { Resilient } \\
\text { (vs.average) }\end{array}$ & $\begin{array}{l}\text { Garden Engagement } \\
\text { Male }\end{array}$ & $\begin{array}{c}1.09 \\
-1.24\end{array}$ & $\begin{array}{l}.63 \\
.72\end{array}$ & $\begin{array}{c}1.74 \\
-1.71\end{array}$ & $\begin{array}{l}.08 \\
.09\end{array}$ & $\begin{array}{l}2.97 \\
.29\end{array}$ & $\begin{array}{c}1.06-8.32 \\
.09-.95\end{array}$ \\
\hline
\end{tabular}

$n=167$. All developmental resources grand-mean centered. Odds ratio indicates the likelihood of being in the selected group as it was for them to be members of the average group with each increased unit of the developmental resource, where 1 is equal, $<1$ is lesser, and $>1$ is greater. 
members of the resilient group as it was for them to be members of the average group, $p=.07$.

Peer support did not appear to serve as either a protective or enabling resource for eighth graders' dual resilience. The combination of peer support and gender did not significantly explain dual resilience group membership, as compared with the intercepts-only model, $X^{2}(4)=6.34, p=.18$.

When controlling for gender, garden engagement was not significant as protective resource against eighth graders' dual stress-affectedness, but was a marginally significant enabling resource. The combination of garden engagement and gender significantly explained dual resilience group membership, as compared with the intercepts only model, support $X^{2}(4)=14.00, p<.007$. When controlling for gender, a one unit increase in garden engagement was marginally associated with being 2.97 times as likely for students to be members of the resilient group as it was for them to be members of the average group, $p=.08$. After controlling for garden, gender was a marginally significant predictor of resilience group, such that being male instead of female corresponded to being .29 times (about one quarter) as likely for students to be members of the resilient group as it was for them to be members of the average group, $p=.09$.

Thus, our hypothesis was partially supported in that three of the four developmental resources predicted students' dual resilience group. As was found in research question 5a, autonomy was a significant predictor of dual resilience group. In contrast to the model from 5a, however, teacher support was also a strong predictor of 
Appendix

both dually resilient and dually stress-affected group membership when not controlling for the other developmental resources, and garden engagement was a marginally significant predictor of dually resilient group membership. Additionally, while the models for teacher support, peer support, and garden engagement all showed a similar marginally significant effect for gender, this effect was not uniquely significant in the model for autonomy.

Sixth graders: when tested separately, are the four developmental resources descriptively stronger unique predictors of dual resilience group membership for eighth-graders than for sixth-graders?

As they did with eighth-grade students, autonomy, teacher support, and garden engagement all predicted dual resilience group when controlling for gender, and peer support did not predict dual resilience group when controlling for gender. Only the models for autonomy, teacher support, and garden engagement demonstrated significantly better fit than intercepts-only models, indicating that the combination of that developmental resource and gender explained significant variance in dual resilience group membership for those models. Regression slopes, $p$-values, standard errors, $z$-scores, odds ratios, and confidence intervals are reported along with model fit information for each model in Table A10.

When controlling for gender, autonomy seemed to serve both as an enabling resource for sixth-grade students' dual resilience and a protective resource against their stress-affectedness, as it did for eighth-grade students. The combination of autonomy and gender significantly explained dual resilience group membership, as 
compared with the intercepts only model, $X^{2}(4)=35.66, p<.001$. When controlling for gender, a one unit increase in autonomy corresponded to being 4.18 times as likely for students to be members of the resilient group as it was for them to be members of the average group, $p=.01$, and .38 times (about one third) as likely for students to be members of the stress-affected group as it was for them to be members of the average group, $p=.001$. Whereas gender was not significant after controlling for autonomy for the eighth graders, gender was marginally significant $(p=.10)$ after controlling for autonomy.

When controlling for gender, teacher support also seemed to serve as both an enabling resource for sixth-grade students' dual resilience and a protective resource against students' dual stress-affectedness. The combination of teacher support and gender significantly explained dual resilience group membership, as compared with the intercepts only model, support $X^{2}(4)=35.34, p<.001$. When controlling for gender, a one unit increase in teacher support corresponded to being 4.57 times as likely for students to be members of the resilient group as it was for them to be members of the average group, $p=.005$, and .23 times (about one quarter) as likely for students to be members of the stress-affected group as it was for them to be members of the average group, $p<.001$. After controlling for teacher support, gender was a significant predictor of stress-affected group membership, such that being male instead of female corresponded to being 3.12 times more likely to be in the stress-affected group as it was for them to be members of the average group, $p=.03$.

Peer support did not appear to serve as either a protective or enabling resource 
Table A10

Developmental Resources as Predictors of Sixth-grade Dual Resilience Group: Multinomial Logistic Regressions.

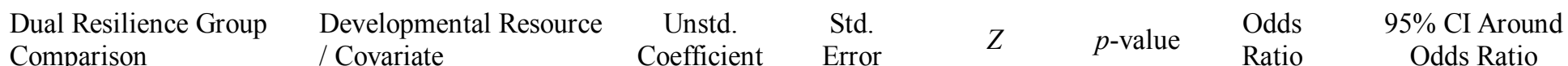

\begin{tabular}{|c|c|c|c|c|c|c|c|}
\hline \multicolumn{8}{|c|}{ 4utonomy, controlling for gender: Loglikelihood $=-405.410$ (11), Scaling factor -.95, AIC $=832.82$, BIC $=866.30$. } \\
\hline \multirow{2}{*}{$\begin{array}{l}\text { Stress-affected } \\
\text { (vs. average) }\end{array}$} & Autonomy & -.98 & .29 & -3.41 & .001 & .38 & $.23-.60$ \\
\hline & Male & .91 & .56 & 1.62 & .10 & 2.49 & $.99-6.26$ \\
\hline $\begin{array}{l}\text { Resilient } \\
\text { (vs.average) }\end{array}$ & $\begin{array}{l}\text { Autonomy } \\
\text { Male }\end{array}$ & $\begin{array}{l}1.43 \\
-.70\end{array}$ & $\begin{array}{l}.58 \\
.55\end{array}$ & $\begin{array}{l}2.48 \\
-1.26\end{array}$ & $\begin{array}{l}.01 \\
.21\end{array}$ & $\begin{array}{c}4.18 \\
.50\end{array}$ & $\begin{array}{c}1.62-10.83 \\
.20-1.23\end{array}$ \\
\hline
\end{tabular}

Teacher Support, controlling for gender: Loglikelihood $=-348.26$ (11), Scaling factor $=.91$, AIC $=718.52$, BIC $=752.00$.

\begin{tabular}{llcccccc}
\hline Stress-affected & Teacher Support & -1.47 & .39 & -3.76 & $<.001$ & .23 & $.12-.44$ \\
(vs. average) & Male & 1.14 & .53 & 2.15 & .03 & 3.12 & $1.31-7.43$ \\
\hline Resilient & Teacher Support & 1.52 & .54 & 2.81 & .005 & 4.57 & $1.88-11.15$ \\
(vs.average) & Male & -.59 & .52 & -1.12 & .26 & .56 & $.24-1.31$
\end{tabular}

Peer Support, controlling for gender: Loglikelihood $=-357.88$ (11), Scaling factor $=.96$, AIC $=737.76$, BIC $=771.24$.

\begin{tabular}{llcccccc}
\hline Stress-affected & Peer Support & .13 & .42 & .30 & .76 & 1.14 & $.57-2.28$ \\
(vs. average) & Male & 1.13 & .51 & 2.23 & .03 & 3.10 & $1.34-7.15$ \\
\hline Resilient & Peer Support & .03 & .33 & .08 & .94 & 1.03 & $.57-1.68$ \\
(vs.average) & Male & .75 & .50 & 1.51 & .13 & 2.11 & $.21-1.07$
\end{tabular}

Garden Engagement, controlling for gende: Loglikelihood $=-359.59$ (11), Scaling factor $=1.04$, AIC $=741.18$, BIC $=774.66$.

\begin{tabular}{llcccccc}
\hline Stress-affected & Garden Engagement & -.53 & .30 & -1.74 & .08 & .59 & $.36-97$ \\
(vs. average) & Male & 1.09 & .51 & 2.12 & .03 & 2.96 & $1.28-6.88$ \\
\hline Resilient & Garden Engagement & 1.35 & .50 & 2.67 & .007 & 3.85 & $1.68-8.82$ \\
(vs.average) & Male & -.84 & .54 & -1.55 & .12 & .43 & $.18-1.05$ \\
\hline
\end{tabular}

Note. $n=155$. All developmental resources grand-mean centered. Odds ratio indicates the likelihood of being in the selected group as it was for them to be members of the average group with each increased unit of the developmental resource, where 1 is equal likelihood, $<1$ is lesser, and $>1$ is greater. 
for sixth-grade students' dual resilience. The combination of peer-support and gender was marginally significant in explaining dual resilience group membership, as compared with the intercepts-only model, $X^{2}(4)=9.43, p=.05$. However, gender was the only significant predictor of dual resilience group membership, such that being male instead of female corresponded to being 3.10 times more likely for students to be members of the stress-affected as it was for them to be members of the average group after controlling for peer support, $p=.03$.

When controlling for gender, garden engagement seemed to serve as both an enabling and a protective resource for sixth-grade students' dual resilience. The combination of garden engagement and gender significantly explained dual resilience group membership, as compared with the intercepts only model, support $X^{2}(4)=21.61$, $p<.001$. When controlling for gender, a one unit increase in garden engagement was significantly associated with being 3.85 times as likely for students to be members of the resilient group as it was for them to be members of the average group after controlling for gender, $p=.007$, and was marginally associated with being .59 times (about one half) as likely for students to be members of the stress-affected group than the average group after controlling for gender, $p=.08$. After controlling for garden engagement, gender was a significant predictor of stress-affected group membership, such that being female instead of male corresponded to being 2.96 times more likely to be in the stress-affected group as it was for them to be members of the average group, $p$ 
$=.03$.

Thus, our hypothesis for the preliminary regressions was not supported. The developmental resources were similar as predictors of dual resilience group membership for both grades, and some relationships (teacher support and autonomy with dual resilience group) seemed to be somewhat stronger for sixth graders than eighth graders.

\section{Research Question 5ba: Further Description of Eighth-grade Mediation Analyses}

\section{Eighth-grade Teacher Support, Stress-affected Group}

In RQ5b1, we saw that teacher support marginally predicted the likelihood of being in the stress-affected group as it was for them to be members of the average group for eighth-graders but that gender did not, so gender was omitted from the mediation analyses. Model fit information and standardized coefficients are reported in Table A11. In the first step, we redefined this model to predict the dichotomized outcome variable of dually stress-affected vs. average group, and saw that teacher support was negatively associated with stress-affected group membership $(\beta=-.35, p=.004)$. In the second step of the analysis, we found that teacher support significantly predicted autonomy ( $\beta$ $=.23, p=.002$.) In the third step, autonomy was negatively associated with dually stress-affected group membership after controlling for teacher support, $(\beta=-.30, p=$ $.02)$. In the fourth step, teacher support was marginally associated with dually stressaffected group membership after controlling for autonomy $(\beta=-.20, p=.07)$. The indirect effect of teacher support to dual resilience group through autonomy was significant, $(\beta=-.07, p=.03)$. This suggests that autonomy partially mediated the 
Appendix

relationship between teacher support and dually-stress-affected group membership for eighth graders.

\section{Eighth-grade Garden Engagement, Resilient Group}

In RQ5b1, we saw that both garden engagement and gender marginally predicted the likelihood of being in the resilient group as it was for them to be members of the average group, so both variables were included in the mediation analyses. Model fit information and standardized coefficients are reported in Figure 5.6. In the first step, we redefined this model to predict the dichotomized outcome variable of dually resilient vs. average group, and confirmed that garden engagement $(\beta=.48, p=.10)$ and gender

$(\beta=-33, p=.08)$ were both marginally positively associated with dually resilient group membership. In the second step of the analysis, we found that garden engagement was not associated with autonomy $(\beta=-.03, p=.72)$ when controlling for gender, and therefore autonomy could not be considered a mediator for the relationship between garden engagement and resilience group. We did find, however, that gender was negatively associated with autonomy $(\beta=-.13, p=.05)$, and continued to investigate whether autonomy mediated the relationship between gender and resilience group. In the third step, autonomy was positively associated with dually resilient group membership after controlling for garden engagement and gender $(\beta=.43 p=.009)$. In the fourth step, gender was no longer significantly associated with resilience group after 
controlling for garden engagement $(\beta=-.24, p=.18)$. The indirect effect of garden engagement to dual resilience group through autonomy, however, was not significant, $(\beta$ $=-.06, p=.11)$.

Thus, these analyses suggest that autonomy did not mediate the relationship between garden engagement and dually resilient group membership, and that evidence was mixed for whether autonomy mediated the relationship between gender and dually resilient group membership.

\section{Research Question 5b2: Further Information on Mediations for Sixth Graders}

In this question, we tested whether autonomy also mediated the relationships between the developmental resources and dual resilience group for sixth graders, with the expectation that autonomy would be a stronger mediator for eighth graders. Mediation analyses were conducted for the developmental resources that demonstrated significant or marginally significant relationships with dual resilience group membership in the preliminary regressions. In those analyses, teacher support predicted both dually resilient and stress-affected group membership, peer support did not predict dual resilience group membership, and garden engagement predicted both duallyresilient and dually stress-affected group membership. Gender was included in the models when it was at least marginally significant in the models for preliminary regressions.

\section{Sixth-grade Teacher Support, Resilient Group}

In the preliminary regressions, we saw that teacher support marginally predicted 
the likelihood of being in the resilient group as it was for them to be members of the average group for sixth graders but that gender did not. Gender was therefore omitted from the mediation analyses. Model fit information and standardized coefficients are reported in Figure 5.7. In the first step, we redefined this model to predict the dichotomized outcome variable of dually resilient vs. average group, and confirmed that teacher support was positively associated with dually resilient group membership ( $\beta=$ $.45, p<.001$. In the second step of the analysis, we found that teacher support significantly predicted autonomy $(\beta=.27, p<.001$.) In the third step, autonomy was positively associated with dually resilient group membership after controlling for teacher support, $(\beta=.50, p=.03)$. In the fourth step, teacher support was marginally associated with dually stress-affected group membership after controlling for autonomy $(\beta=.25, p=.07$.$) The indirect effect of teacher support to dual resilience group through$ autonomy was significant, $(\beta=.14, p=.04)$. This suggests that autonomy partially mediated the relationship between teacher support and dually-resilient group membership for sixth graders.

\section{Sixth-grade Teacher Support, Stress-affected Group}

In the preliminary regressions, we saw that both teacher support and gender significantly predicted the likelihood of being in the stress-affected group rather than the average group, so both variables were included in the mediation analyses. Model fit information and standardized coefficients are reported in Figure 5.7. In the first step, we redefined this model to predict the dichotomized outcome variable of dually stress- 
affected vs. average group, and confirmed that teacher support was negatively associated with dually stress-affected group, $(\beta=-.49, \mathrm{p}<.001)$, and that gender was positively associated with dually stress-affected group, $(\beta=.29, \mathrm{p}=.05)$. In the second step of the analysis, we found that teacher support was positively associated with autonomy $(\beta=.34, \mathrm{p}=<.001)$, and that gender was not associated with autonomy $(\beta=$ -

$.02, \mathrm{p}=.61)$. In the third step, autonomy was significantly associated with dually stressaffected group membership after controlling for teacher support, $(\beta=-.33, \mathrm{p}=.05)$. In the fourth step, teacher support was marginally associated with dually resilient group membership $(\beta=-.27, \mathrm{p}=.06)$, after controlling for autonomy and gender $(\beta=.25, \mathrm{p}=$ .07). The indirect effect of teacher support to dually resilient group through autonomy was significant, $(\beta=-.11, \mathrm{p}=.05)$. Thus, there is evidence that the relationship between teacher support and dually resilient group membership was partially mediated by autonomy, whereas autonomy appeared to fully mediate that relationship for eighth graders.

\section{Sixth-grade Garden Engagement, Resilient Group}

In the preliminary regressions, we saw that garden engagement significantly predicted the likelihood of being in the resilient group rather than the average group for sixth graders but that gender did not. Gender was therefore omitted from the model. Model fit information and standardized coefficients are reported in Figure 5.8. In the first step, we redefined this model to predict the dichotomized outcome variable of dually resilient vs. average group, and confirmed that garden engagement was 
positively associated with dual resilience group membership $(\beta=.43, p=.003)$. In the second step of the analysis, we found that garden engagement significantly predicted autonomy ( $\beta=.18, p=.004$.) In the third step, autonomy was positively associated with dually resilient group membership after controlling for garden engagement $(\beta=$ $.51, p=.01)$. In the fourth step, garden engagement was still significantly associated with dually resilient group membership after controlling for autonomy $(\beta=.27, p=$ .04.) The indirect effect of garden engagement to dually resilient group membership through autonomy was marginally significant, $(\beta=.09, p=.06)$. This suggests that autonomy partially mediated the relationship garden engagement and dually-resilient group membership for sixth graders, whereas autonomy was not a mediator of garden engagement for eighth graders.

\section{Sixth-grade Garden Engagement, Stress-affected Group}

In the preliminary regressions we saw that both garden engagement and gender significantly predicted the likelihood of being in the stress-affected group rather than the average group, so both variables were included in the mediation analyses. In the first step, we redefined this model to predict the dichotomized outcome variable of dually

stress-affected vs. average group, and confirmed that garden engagement was marginally associated $(\beta=-.21 \mathrm{p}=.09)$, and gender was significantly associated $(\beta=$ $.28, p=.04)$, with dually stress-affected group. In the second step of the analysis, we found that garden engagement was positively associated with autonomy $(\beta=.23, \mathrm{p}=$ $<.001)$, but that gender was not associated with autonomy $(\beta=-.04, \mathrm{p}=.42)$. In the 
third step, autonomy was significantly associated with dually stress-affected group membership $(\beta=-.48, p=.003)$ after controlling for garden engagement. In the fourth step, garden engagement was no longer associated with dually stress-affected group membership $(\beta=-.01, p=.91)$, after controlling for autonomy and gender $(\beta=.23, p=$ $.07)$. The indirect effect of garden engagement to dually stress-affected group through autonomy was significant, $(\beta=-.11, p=.02)$. Thus, there is evidence that the relationship between garden engagement and dually stress-affected group membership was fully mediated by autonomy. Because garden engagement did not significantly predict eighth-grade dually stress-affected group membership for eighth graders (and because garden engagement and autonomy were not significantly correlated for eighth graders), this relationship was not investigated for eighth graders.

\section{Summary of RQ5b}

In general, teacher support seemed to be partially mediated by autonomy for both grades, whereas garden engagement was mediated by autonomy for sixth graders and not eighth graders. When taken in consideration with the findings from the preliminary regressions that, for eighth-graders, autonomy was the only unique predictor of dual resilience group, but that teacher support was a stronger unique predictor for sixth graders, there seems to be support for the idea of autonomy as an important unique pathway to motivational and academic resilience for eighth-grade students. Autonomy did also appear to be an important unique pathway for sixthgraders, but when considered in conjunction with the findings from Rq5a and the 
preliminary regressions, teacher support seemed to be a more important unique pathway than autonomy for those students.

For both eighth graders and sixth graders, the relationships between teacher support and dual resilience group were at least partially mediated by autonomy. There was weak evidence that the relationship between eighth-grade teacher support and dually resilient group membership was mediated more fully for the eighth graders. In research question 5a, teacher support was a strong unique predictor of dual resilience group for sixth graders, after controlling for autonomy, but not for eighth graders. Considering these findings in conjunction, it does appear that autonomy might serves more of a central and necessary role in motivational and academic resilience for eighthgraders than for sixth graders. That is, perhaps for eighth graders the helpfulness of teacher support depends upon its capacity to help students identify with or care about their schoolwork.

Garden engagement was unequivocally not mediated by autonomy for eighth graders, lacking the significant association with autonomy that would make mediation possible. For sixth graders, however, the relationship between garden engagement and dually resilient group was partially mediated, and the relationship between garden engagement and dually stress-affected group was the only instance in this series of analyses of a true full mediation, in that when autonomy was added to the prediction equation the direct effect was no longer significant, but appeared to completely negate the size of the effect (from -.21 to --.01.) This lends more support to the idea that, even 
though it showed no unique effects garden engagement and the purposeful academic work it provides might provide a separate pathway to motivational and academic resilience for eighth graders and not sixth graders that is unrelated to autonomy.

Finally and unexpectedly, autonomy seemed to mediate the relationship between gender and dual resilience group membership for eighth-graders, both in the preliminary regressions, with autonomy and gender alone, and in the model of autonomy as a mediator between garden engagement and dually resilient group. At the same time, gender was generally not associated with autonomy for sixth graders in the meditational models. This could suggest a link between gender and academic identity in eighthgraders: Perhaps in eighth grade, part of what is academically and motivationally enabling about being female or (harmful about being male) has to do with one's autonomy as it corresponds to a sense of ownership and identity with school. 


\section{APPENDIX E}

\section{NOTES ON GENDER}

Table A11

Summary of Relationships of Developmental Resources with Motivation and Achievement with Notations when Results Varied by Gender.

$\frac{\text { Autonomy }}{8^{\text {th }} 6^{\text {th }}} \quad \frac{\begin{array}{c}\text { Teacher } \\ \text { Support }\end{array}}{8^{\text {th }} 6^{\text {th }}} \quad \frac{\text { Peer Support }}{8^{\text {th }} 6^{\text {th }}} \quad \frac{\begin{array}{c}\text { Garden } \\ \text { Engagement }\end{array}}{8^{\text {th }}} 6^{\text {th }}$

Correlations:

Significant and positive?

\begin{tabular}{lllllllll}
\hline Engagement & Yes & Yes & Yes & Yes & Yes $^{\boldsymbol{m}}$ & -- & Yes & Yes \\
GPA & Yes & Yes & Yes & Yes $^{f}$ & -- & -- & Yes $^{f}$ & Yes
\end{tabular}

ANOVA Results:

Enabling factor, General resource (resilient $>$ stressed), and/or protective factor?

\begin{tabular}{|c|c|c|c|c|c|c|c|c|}
\hline $\begin{array}{l}\text { Motivational } \\
\text { Resilience }\end{array}$ & $\begin{array}{l}\text { Enab. } \\
\text { Gen. } \\
\text { Prot. }\end{array}$ & $\begin{array}{l}\text { Enab. } \\
\text { Gen. } \\
\text { Prot. }\end{array}$ & $\begin{array}{l}\text { Enab. } \\
\text { Gen. } \\
\text { (Prot.) }\end{array}$ & $\begin{array}{l}\text { Enab. } \\
\text { Gen. } \\
\text { Prot. }\end{array}$ & $\begin{array}{l}-- \\
\text { Gen. } \\
\text { m }\end{array}$ & $\begin{array}{l}-- \\
-- \\
--\end{array}$ & $\begin{array}{l}\text { (Enab) }{ }^{f} \\
\text { Gen. }{ }^{f} \\
--\end{array}$ & $\begin{array}{l}- \\
\text { Gen. } \\
--\end{array}$ \\
\hline Academic Resilie & $\begin{array}{l}\text { (Enab) }^{f} \\
\text { Gen. }^{f}\end{array}$ & $\begin{array}{l}-- \\
--\end{array}$ & $\overline{-}-\overline{\text { Gen. }}$ & $\begin{array}{l}-- \\
--\end{array}$ & $\begin{array}{l}-- \\
--\end{array}$ & $\begin{array}{l}-- \\
--\end{array}$ & Gen. $^{f}$ & $\begin{array}{l}-- \\
--\end{array}$ \\
\hline Dual Resilience & $\begin{array}{l}\text { (Enab.) } \\
\text { Gen. } \\
\text { Prot. }\end{array}$ & $\begin{array}{l}\text { Enab. } \\
\text { Gen. } \\
\text { Prot. }\end{array}$ & $\begin{array}{l}\text { (Enab. } \\
\text { Gen. } \\
\text { Prot. }\end{array}$ & $\begin{array}{l}\text { Enab. } \\
\text { Gen. } \\
\text { Prot. }\end{array}$ & $\begin{array}{l}-- \\
-- \\
--\end{array}$ & -- & $\begin{array}{l}- \\
\text { Gen. }{ }^{f} \\
--\end{array}$ & $\begin{array}{l}\text { Enab. } \\
\text { Gen. } \\
--\end{array}$ \\
\hline
\end{tabular}

Regression Results:

Enabling factor and/or protective factor?

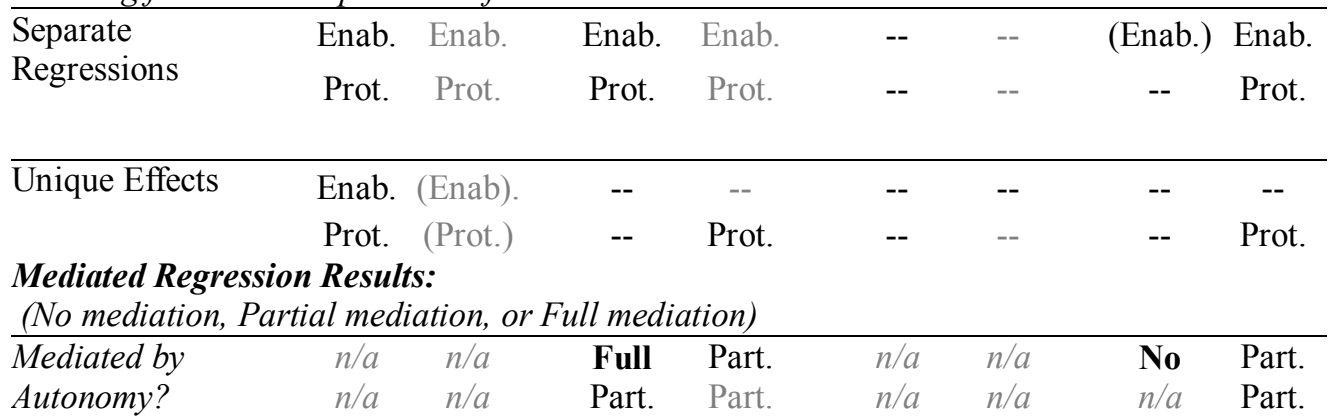

Note: Superscript of "f " or " $m$ " implies that, when male and female students were analyzed separately, correlation was only present for female $\left({ }^{f}\right)$ or male $\left({ }^{m}\right)$ students. Eighth-grade results bolded when differing from sixth-grade results, sixth-grade results in grey unless different than eighth-grade results. Parentheses indicate result was only marginally significant. 